\title{
The Triple Burden of War : An assessment of the mental health status and coping strategies of Sudanese female undergraduates for developing a psychosocial counsellor training program
}

Citation for published version (APA):

Badri, A. (2013). The Triple Burden of War : An assessment of the mental health status and coping strategies of Sudanese female undergraduates for developing a psychosocial counsellor training program. [Doctoral Thesis, Maastricht University]. Universitaire Pers Maastricht. https://doi.org/10.26481/dis.20130314ab

Document status and date:

Published: 01/01/2013

DOI:

$10.26481 /$ dis.20130314ab

Document Version:

Publisher's PDF, also known as Version of record

Please check the document version of this publication:

- A submitted manuscript is the version of the article upon submission and before peer-review. There can be important differences between the submitted version and the official published version of record. People interested in the research are advised to contact the author for the final version of the publication, or visit the DOI to the publisher's website.

- The final author version and the galley proof are versions of the publication after peer review.

- The final published version features the final layout of the paper including the volume, issue and page numbers.

Link to publication

\footnotetext{
General rights rights.

- You may freely distribute the URL identifying the publication in the public portal. please follow below link for the End User Agreement:

www.umlib.nl/taverne-license

Take down policy

If you believe that this document breaches copyright please contact us at:

repository@maastrichtuniversity.nl

providing details and we will investigate your claim.
}

Copyright and moral rights for the publications made accessible in the public portal are retained by the authors and/or other copyright owners and it is a condition of accessing publications that users recognise and abide by the legal requirements associated with these

- Users may download and print one copy of any publication from the public portal for the purpose of private study or research.

- You may not further distribute the material or use it for any profit-making activity or commercial gain

If the publication is distributed under the terms of Article 25fa of the Dutch Copyright Act, indicated by the "Taverne" license above,

Download date: 26 Apr. 2023 


\section{The Triple Burden of War}

An assessment of the mental health status and coping strategies of Sudanese female undergraduates for developing a psychosocial counsellor training program 
The research project presented in this thesis was conducted under the auspices of the School for Public Health and Primary Care: CAPHRI, Department of Health Promotion, at Maastricht University, The Netherlands. CAPHRI participates in the Netherlands School of Primary Care Research CaRe, and has been acknowledged since 1995 by The Royal Netherlands Academy of Art en Sciences (KNAW). CAPHRI was classified as 'excellent' by the external evaluation committee of leading international experts that reviewed CAPHRI in December 2010.

Cover Picture: Alia Badri

Layout and Printing: Datawyse | Universitaire Pers Maastricht

ISBN 9789461592132

(C) Alia Badri, Maastricht 2013

All rights reserved. No part of this publication may be reproduced, or transmitted in any form or by any means, electronic or mechanical, including photocopying, recording, or any information storage and retrieval without prior permission from the author, or, when appropriate the publishers of the papers. 


\title{
The Triple Burden of War
}

An assessment of the mental health status and coping strategies of Sudanese female undergraduates for developing a psychosocial counsellor training program

\author{
Dissertation \\ to obtain the degree of Doctor at Maastricht University, \\ on the authority of the Rector Magnificus, Prof. dr. L.L.G. Soete \\ in accordance with the decision of the Board of Deans, to be defended in public on \\ Thursday March 14, 2013, at 10.00 hours.
}

by

Alia Badri

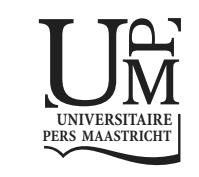




\section{Promotor}

Prof. dr. H. W. van den Borne

\section{Copromotor}

Dr. Rik Crutzen

\section{Assessment committee}

Prof. Dr. N.K. de Vries (chair)

Prof. dr. G. J. Kok

Prof dr. B. Mesquita (University of Leuven, Belgium)

Prof. dr. P. Muris

Dr. A. Basit M. Musa (Gazera University, Sudan) 


\section{DEDICATIONS}

To the soul of my late father, Dr. Babiker Ali Badri, who has shown me nothing but encouragement, dignity, distinction and love. Much appreciated Dad.

To my mother, Nafisa Yousif Badri who continues to provide unending support and has seamlessly and selflessly endowed me with decorum, and respectability that has endorsed all that I am today. May long shall she live in health and properity. I love you Mummy.

To Isam, my beloved husband, and Meccawi, Mukhtar and Joud our cherished children, who have put up with my travels, absences and absent-mindedness throughout this journey, only to return them with patience, love and tolerance. Thanks guys. 


\section{ACKNOWLEDGEMENTS}

Like everyone else who has had the good fortune of working with Professor Dr. Bart van den Borne, I owe my humblest and deepest gratitude to his unfailing support, indispensible suggestions, and constant corroboration in the completion of this project.

Since the genesis of this project, you have listened intently to the nature and culture of the Sudanese society and Darfuri tradition. Your full immersion in this context has guided me on this journey of learning and allotted me with a unique blend of freedom, independence and challenge to pursue methodological designs in positivistic ways.

On a personal level Professor Bart has been an inspirational part of our family, nuclear and extended, to which we shall remain loyal.

I will always endeavour to be in your favour.

I am indebted to Dr. Rik Crutzen for his brilliance in the field of methodology and jungle of statistics. I am indebted to your elegant teachings and coaching of SPSS and statistical terms and facts. Your expertise has been a cornerstone in the completion of this project. I am in deep awe and respect for your agility and speed in grasping the philosophy, nature and culture in which this project is immersed. Your timely comments and corrections have seen the fruition of each and every article throughout this project. I am so grateful for your time in editing, formatting and constructing this thesis.

I thank you for your consent to be my co-promoter.

I am obliged to Professor Dr. Balghis Badri who has once again lived up to her excellent reputation as supervisor. Your reliable and responsible attention to detail motivated and inspired me to pay more attention to the socio-political, historical and cultural issues imbedded in this project. Your valuable and respectful advice that was so readily in supply irrefutably allotted the unerring precision with which to reach this project's completion.

I shall remain forever in your debit.

I would like to thank Professor Dr. Gasim Badri who in his unassuming manner has provided me with endless encouragement and faith in my humble accomplishments throughout my life.

Your fatherly support in all my endeavours from undergraduate study at AUW through to Masters at Warwick in the UK and now the completion of this $\mathrm{PhD}$ is a true testament that your love knows no bounds. I am also grateful for your absolute conviction and certitude that only the best will do for our female Sudanese students is contagious.

I hope to always meet your expectations. 
I extend my gratitude and acknowledgement to Dr. Shahla ElTyeb for sharing her time and commitment.

You have been an inspiration since the initial conception of this research project. Your invaluable discussions, advice and suggestions throughout each phase, and the unfailing assistance you provided within each article have led to the successful and timely completion of this mission. As senior organizer of the field staff data collectors you have seamlessly and vigilantly carried out this task. I could not have carried out this research project without your contemporary insight and relevant contextual knowledge on the issues within AUW. Your persistence, camaraderie and mutual respect furnished the ultimate completion of this work.

May we continue to strive for excellence.

I offer great thanks and appreciation to Marja and Leon for their continued technical and logistical support and ideas. I thank you both for welcoming me warmly every July to the Maastricht family. May our friendship continue for many years to come.

I am indebted to the students of Ahfad University for Women, especially the Darfuri participants, who despite their ordeal and suffering were willing to participate in the interviews and survey and completed the arduous questionnaire.

To my Aunt Aziza Mukhayer and Uncle Mohamed Mukhayer, I thank you dearly for your generosity and hospitality in providing accommodation throughout my study in Maastricht. A special acknowledgement of gratitude to you, Aziza, for your unfailing support throughout these years in sharing your time, books (Andy \& step-by-step!) and views. I appreciate your persistent encouragement with your kind touch and soft words. To my younger sister Amouna, Nafos and Madon, we shared great times and I cannot thank you enough for giving up your space and time to accommodate mine. May God bless you all.

My husband, Isam. No linguistic expressions of gratitude are adequate. Suffice it to say that You are my foundation and my rock. I owe more than a 'thank you' for your respect and unfailing love, support and generosity throughout this journey. Thank you for being my second set of eyes as you meticulously and thoroughly read and reviewed each chapter, elevating the language to a more elegant state. Thank you for accepting the daunting duty of translating the Summary into Arabic; a painstaking task! I thank you most especially for being you, a true 'gentle-man'.

To Joud, Mukhtar and Meccawi, I am proud of your maturity and understanding for your sacrifice throughout these years and offer my eternal love and appreciation.

Several people deserve special thanks for their dedication and contribution in the data collection process: Dr. Amira Y. Badri, Khalid Alshafee, Ikhlas Abbas and Arig Badri. 
I am also grateful to the department administrators and school secretaries for allowing us to conduct this research in their classrooms.

I am especially indebted to Dr. Babiker Ahmed Badri whose insight into the social aspects of Sudanese life and Darfuri needs was invaluable.

I would like to thank Dr. Ayman Badri and Dr. Osama Awad for their great contribution in providing the necessary logistics and important information relevant to the AUW student body.

I extend my appreciation to the contribution of the translation committee, Mr. Hafiz AbdelMalik, Ms. Salwa Akrat, and Mr. Bakri Alkhier.

And firstly and finally, I thank Almighty God, whom I worship through learning and research. 


\section{Table of Contents}

Chapter 1 General introduction $\quad 11$

1.1. The Sudan: Demography, topography and antiquity 12

1.2. $\quad$ Exposures to war-related traumatic events 15

1.3. Psychological impact of war-related traumatic events 16

1.4. Psychosocial impact of war-related and post-displacement stressors 19

1.5. Coping strategies with war-related trauma and post-displacement 20 stressors

1.6. Impact of ethnic minority background and low SES on adjustment 21

1.7. Coping strategies and resilience among female university students 22

1.8. Exploring mental health and psychological interventions in the Sudan 23

1.9. Dissertation Outline 26

Chapter 2 Experiences and psychosocial adjustment of Darfuri female students 29 affected by war: An exploratory study

Chapter 3 Exposures to war-related traumatic events and post-traumatic stress $\quad 45$ disorder among Darfuri female university students: An exploratory study

Chapter 4 Anxiety, depression and resilience among war-affected and non-war- $\quad 61$ affected Sudanese female university students: A comparative study

Chapter 5 Coping resources and resilience characteristics among war-affected and non-war-affected Sudanese female university students: A comparative study

Chapter 6 Promoting Darfuri women's psychosocial health: Developing a wartrauma counsellor training program

$\begin{array}{ll}\text { Chapter } 7 \text { General Discussion } & 105\end{array}$

7.1. $\quad$ Main Results 108

7.2. Promoting Darfuri female Psychosocial Health 114

7.3. General Remarks of Key Findings 118

7.4. Study Limitations 119

$\begin{array}{ll}\text { 7.5. The Way Forward } & 120\end{array}$

$\begin{array}{ll}\text { 7.6. } & \text { Concluding Remarks } \\ & 122\end{array}$

Summary in English $\quad 123$

Summary in Arabic $\quad 129$

$\begin{array}{ll}\text { Appendices } & 132\end{array}$

Bibliography 153

$\begin{array}{ll}\text { About the Author } & 166\end{array}$ 



\section{Chapter 1 \\ General Introduction}

"The ink of the scholar is more holy than the blood of the martyr."

Prophet Mohamed (PBUH) 
The investigation of mass war-related trauma and post displacement stressors is a topic that has been around since the beginning of modern warfare. The African continent has seen its fair share of misery in that regard and the Sudan is no stranger to the psychosocial effects left behind by many civil wars on its civilians.

The research reported in this dissertation is however unique to one target group; it focuses on university-aged women, distinctive in current affairs; covers the on-going Darfuri conflict, making it current to the horrendous impact of warfare; and pioneer in action oriented research in the Sudan, applying evidence-based knowledge as it assembles a war-trauma counselor training program, concentrating on mental and psychosocial alleviation of war-related distress symptoms.

The introduction of this dissertation will set the stage by transforming us into the world of the Sudan, its demography and topography, its antiquity, the history of its civil wars, and the genesis of the current crisis in Darfur focusing on the repercussions for its citizens. The picture is expanded to include exposures to war-related traumatic events, the consequences of displacement, and the mechanisms by which internally displaced people (IDPs) cope with and adjust to the aftermath of war throughout the African continent and the world at large.

But Sudan's complexity as pertaining to psychosocial and mental health does not stop with the case of war-related trauma and displacement as with the case of its evergrowing IDP situation. There is another dimension to this story; it is the impact of stress and cultural conflict among ethnic minorities and low socioeconomic status (SES) female university students. The introduction will cover the triggers of these factors and the coping strategies and resources used, and resilience levels and characteristics among undergraduates on a global scale.

With the ultimate aim of promoting psychosocial health amongst war victims in the wake of current conflict, this introduction will sketch the contribution made by theory and evidence-based practice in the design and implementation of war-trauma counselor training programs addressed to alleviate and address the mental health needs and concerns within war-affected populations worldwide.

\subsection{The Sudan}

\section{Demography and topography}

The Sudan is a state in the north-eastern region of Africa with an area of 2,505,810 $\mathrm{km}^{2}$, it is the largest country in Africa and the Arab world, and tenth largest in the world. Bordered by Egypt to the north, the Red Sea along nearly 853km of the north-eastern border, Eritrea and Ethiopia to the east, Kenya and Uganda to the southeast, the Democ- 
ratic Republic of the Congo and the Central African Republic to the southwest, Chad to the west and Libya to the northwest.

The terrain is generally flat plains, mainly dry arid deserts to the north (Nubian Desert) and swampy rainforest to the south. Several mountain ranges break up the plains; in the west the Jebel Marra is the highest range; in the south is the highest mountain Mount Kin yeti Imatong $(3,187 \mathrm{~m} / 10,456 \mathrm{ft})$, near the border with Uganda; in the east are the Red Sea Hills. The Blue and White Niles meet in Khartoum to form the world's longest river, the Nile, dividing the country between east and west flowing northwards through Egypt to the Mediterranean Sea. Some $800 \mathrm{~km}$ long of significant tributaries such as the Dinder and Rahad rivers run between Sennar and Khartoum making up the main irrigation course and most fertile land on earth.

Straddling the cultural and geographical divide of two distinct historical and ethnical cultures, the Sudan's demography portrays both Arab and African tradition. With 578 tribes consisting of smaller ethnic divisions and over 145 different languages including Nubian, Beja, Fur, and Hausa, and 400 dialects including Nuban and Niolotic, myriad physical appearance, traditional custom, and religious affiliation engulfs the nearly 40 million Sudanese.

\section{The Sudan's antiquity}

Historically, the Sudan dates back to some eight thousand years BC, during the Kushite and Meroitic Kingdoms and the Pharaonic Taharqa and Napata Dynasties. Since then the Ottoman rule (1821-1885), the Mahadist rule (1885-1899), and the Anglo-Egyptian Condominium (1899-1956) shaped today's Sudanese tribal, ethnic, clanship, religious and even political portrait. Shortly after gaining independence from Egypt and the United Kingdom in 1956, civil war and a succession of military coups and dictatorships have tainted the antiquity of the Sudan.

\section{The History of Civil War}

From 1924, it was illegal for people living north of the 10th parallel to go further south and for people south of the 8th parallel to go further north. The law was ostensibly enacted to prevent the spread of malaria and other tropical diseases that had ravaged British troops, as well as to facilitate spreading Christianity among the predominantly Animist population while stopping the Arabic and Islamic influence from advancing south. The result was increased isolation between the already distinct north and south and arguably laid the seeds of conflict in the years to come.

Dominated by a starkly divided country on racial, religious, regional, and economic grounds the Sudan's independent history has suffered chronic and exceptionally cruel warfare between the Northern Arab and Nubian Sudanese and the African Nilotes of Southern Sudan for more than 40 years $(1956-1977 ;$ 1983-2005). Peace talks between 
the southern rebels (SPLM/SPLA) and the government made with a lot of pressure from the international community, substantial progress in 2004, with the signing of the landmark Comprehensive Peace Agreement in January 2005. Granting the Southern Sudan autonomy followed by a referendum for independence, which was almost unanimously voted for, cessation from the larger Sudan Republic in July 2011. The combination of civil war, famine, and displacement has taken the lives of nearly 2 million people in Southern Sudan and displaced more than 6 million refugees.

\section{The genesis of the current crisis in Darfur}

The Arabic word Dar roughly means homeland, and its population of nearly four million is divided into several 'Dars'; not only of the Fur people, as its name suggests, but also of several other communities, determined by livelihood as much as ethnicity. Two main ethnicities reside in Darfur: African and Arab tribes. The Fur, Berti, Bargu, Bergid, Tama and Tunjur and Massaleet African tribes occupy the central belt of the region, including the Jebel Marra, the richest area in terms of soil fertility and water resources. The northernmost zone is Dar Zaghawa, inhabited principally by the non-Arab tribes of Zaghawa, Bedeyat, Arab Mahariya, Irayqat, Mahamid and Beni Hussein. It is an ecologically fragile zone and most acutely affected by drought, whose occupants are mainly camel nomads. Cattle rather than camels are herded by the Arab nomads of the eastern and southern zone of Darfur, who comprise the Rizaigat, Habbaniya, Beni Halba, Taaisha and Maaliyya. Their area is highly sensitive to fluctuations in rainfall and is also less ecologically stable.

While ethnic diversity exists between the African and Arab tribes on the one hand and traditional distinctions of livelihoods (cattle or camel herders and settled farmers) on the other, history shows that the people of Darfur were prone to tribal skirmishes as well as inter-marriage, both enabling the maintenance of Darfuri social fabric and serving as a basic cornerstone for acceptance and tolerance between the tribes of Darfur (De Waal, 2005). Issues currently propagated in the headlines since the outbreak of major violence in the province and seen largely through the prism of tribalism and pastoral conflict as being central reasons for the current crisis is to make a mockery of the lives lived by both Arab and African in the region; surely the conflict in Darfur is multifaceted.

\section{Repercussions of the Darfuri war on its citizens}

Preceded by droughts and famines in 1983/84, depleting natural resources, loss of livestock, food insecurity and failed livelihoods inundated the region as a whole. Continuous economic marginalization, institutionalized discrimination, oppression and discrimination led in turn to ferocious and brutal tribal battles between Fur tribes of farmers-turned-guerrillas and the Government of Sudan (GOS) armed Arab tribes (generically known as the Janjaweed defined as the devil on horseback) between 1987-1990 
(Elnur, 1994). The nature of attacks left many with lost property and the deaths of family members: a great deal of damage was done (De Waal, 2005).

Since early 2003, Darfur has seen increased and indiscriminate attacks on its villages which not only killed and injured civilians, looted homes, demolished infrastructure, and destroyed property and farmlands, but also led to a widening gap between Arab and African citizens (De Waal, 2009). As the war ebbed and flowed, and with the persistent threat to the safety and security of civilians in present day Darfur who have been burdened by hopeless disposition, an estimated one million were killed and approximately 2.74 million displaced (De Waal, 2009; United Nations, 2005) crossing the border to Chad as refugees (Rasmussen, et al., 2010) or journeying eastwards some $1,200 \mathrm{~km}$ to Omdurman city, as IDPs.

\subsection{Exposures to war-related traumatic events}

Mass trauma and displacement of a civilian population are hallmarks of modern-day warfare. IDPs are technically not a legally recognized entity within global definitions of war-related and man-made disasters as much as refugees are. This fact reflects one of the major difficulties in identifying IDP needs accurately and providing much needed assistance. Consequently they remain near violent situations for longer periods of time as opposed to refugees and their needs go unacknowledged. Displacement thus becomes in itself a direct major traumatic experience for many IDPs around the world.

The American Psychiatric Association (2000) Diagnostic and Statistical Manual of Mental Disorders (DSM IV-TR), defines a traumatic event as one in which the person experienced, witnessed, or was confronted with an event, or events, that involve actual or threatened death, or serious injury, or threat to the physical integrity of the person or others. This conception suggests that the individual, regardless of cultural environment can suffer, in some way, when confronted with overwhelming experiences. Mostly nonWestern people exposed to the effects of war and trauma do not go through these events as individual experience: generally as collective experiences in which the traumatic content of the experience is not attached to the event itself but to the pain and negative consequences associated with it (Summerfield, 1995). In this way, traumatized people from societies where the individual is conceived as a part of an extended family, group, or community trauma is conceptualized more broadly to include the loss or disintegration of cultural beliefs and values. Research suggests that the characteristics and nature of traumatizing events (Herman, Russell, \& Trocki, 1986; Roth, Wayland, \& Woolsey, 1990) and their cultural interpretation (Cánive \& Castillo, 1997) demonstrates the validation for conceptualizing trauma more broadly.

Basoglu and colleagues (Basoglu, Paker, Ozmen, Tasdemir, \& Sahin, 1994) showed that in situations of interrogation, characterized by severe threat and danger, symptoms 
such as hyper-arousal, hyper-vigilance, enhanced auditory acuity and restlessness, are experienced by many IDPs, as well as physical abuse, torture, harassment, forced removals and disappearances (Ellis, MacDonald, Lincoln, \& Cabral, 2008).

In an attempt to flee the war zone, the colossal effects of the war are evident firstly from a material and physiological standpoint: lack of shelter, food, sanitation and hygiene, and access to clean water (Carballo et al., 2004). Components of war-related trauma that IDPs endure usually may include aerial bombardment, mortar fire, attack on house or village, crossfire, burning of houses, loss of possessions and destruction of property, detained, held hostage (Adjukovic, 1998; De Jong et al., 2001; Macksoud \& Aber, 1996; Moisander \& Edston, 2003; Thapa \& Hauff, 2005).

The study of Sudanese communities affected by civil-war is not a new topic: the fifty plus years of civil war and strife in the southern regions of the nation has produced extensive research into the plight of war-related traumatic experiences. Witnessing or experiencing beatings/torture, harassment by armed personnel, robbery/extortion, forced separation from family members, social deprivation, verbal abuse, threats against person, false accusations, and witnessing simulated executions, are all examples of reports and testimonies from Southern Sudanese survivors of war (Krishnan et al., 2004; Neuner et al., 2004; Peltzer, 1999). Particularly brutal effects on Southern Sudanese women in the form of rape or sexual abuse, forced prostitution/sexual slavery, forced circumcision, and other forms of sexual abuse, reported that $44 \%$ witnessed the murder of family or friends, $48 \%$ had directly experienced a combat situation, $22 \%$ had been forcefully separated from family and friends, $15 \%$ beaten or tortured, $10 \% \mathrm{im}$ prisoned, and 8\% raped (Roberts, Damunda, Lomoro, \& Sondrop, 2009).

The IDP situation within Darfur-based camps has brought with it an onslaught of misery where the deprivation of food, water, or medical needs, is of paramount concern. The death of a parent/s, witnessed shooting, humiliation and degradation (e.g., forced nakedness in front of family), stress to senses (e.g., being bound; electric shock), being forced to hide or confined to the camp because of danger outside, seeing others raped or sexually assaulted or forced to witness the abuse of others is also a frequent report (Kim, Torbay, \& Lawry, 2007; Morgos, Worden, \& Gupta, 2008; Rasmussen, Smith, \& Keller, 2007).

\subsection{Psychological impact of war-related traumatic events}

The continuing exposure to war-related traumatic events was first recognized as a range of psychological reactions following the devastating effects of war experiences on soldiers serving in Vietnam. The diagnosis for persons experiencing feelings of fear, sadness, guilt and anger, significant distress or impaired functioning including depression and anxiety as the psychological sequelae of war-related trauma was termed post- 
traumatic stress disorder (PTSD). PTSD became a condition applied to all those who experienced exposure to a life threatening event that is processed in such a way as to produce a sense of current threat (Ehlers \& Clark, 2000). This leads to the experience of symptoms, which fall into three clusters that define PTSD: intrusive symptoms, often involving intrusive thoughts and emotions about the traumatic events, avoidance, emotional numbing and/or symptoms of over arousal (American Psychological Association, 2000; World Health Organisation, 1992).

The American Medical Association (AMA) defines post-traumatic stress disorder, or PTSD, as "a response to an event that involves actual or threatened death, serious injury or other threat to one's physical integrity which must involve intense fear, helplessness or horror." According to the AMA, there are three categories of PTSD, based on the time duration between the traumatic event and symptoms. First, acute PTSD, when symptoms begin in fewer than three months; secondly, chronic PTSD, which occurs when the symptom last three months or longer; and thirdly, PTSD with delayed onset, which occurs after six months have passed between the event and the onset of symptoms.

The prevalence rates of PTSD found on site following war trauma appear to vary considerably depending on the methodology employed by the study. Cardozo et al. (2000) and Cardozo et al. (2003) investigated the mental health of Kosovar Albanians immediately following the war in Kosovo and one year after the war had finished. They found PTSD prevalence rates of $17.1 \%$ and $25 \%$ respectively. Similarly, Gavrilovic et al. (2002) measured PTSD in Yugoslavian students one year after air attacks and found a prevalence rate of only $11 \%$. Scholte et al. (2004) found a prevalence rate of $20.4 \%$ for current PTSD following war and repression in Eastern Afghanistan whereas Somasundaram and Sivayokan (1994) found 27\% following the war in Sri Lanka. De Jong et al. (2001) measured life-time PTSD in four post-conflict settings and found levels of 37.4\% in Algeria, 28.4\% in Cambodia 15.8\% in Ethiopia and 17.8\% in Gaza. Bramsen and Van der Ploeg (1999) on the other hand, assessed for PTSD in a sample of civilian Dutch survivors of world war two 50 years after the end of the war. They found that only $4 \%$ had current PTSD, which may have been because after such a long period the strongest survived. Two studies, both using self-report measures, found higher rates of current PTSD. Cardozo et al. (2004) found 42\% looking at mental health in post-war Afghanistan, whereas Abu-Saba (1999) found 75\% among Beirut students who had been exposed to a high number of traumatic war events.

Prevalence rates for PTSD may vary between these countries and cultures (Moisander \& Edston, 2003). High variability in findings across studies makes it difficult to ascertain the true prevalence of PTSD in civilian survivors of war trauma. This variability could be due to the form of sampling (Green, 1996), or the diverse experiences of trauma, the quantity and severity (Mollica et al., 1998), the passing of time since the trauma experience, and the shift from one definition of pathology to another (DSM III-R 
to DSM IV). However, the focus of the concept of PTSD as a psychological effect of war has been successfully applied in assessment and treatment following many types of traumatic experience and is most commonly studied because of its comprehensibility and its instrumentation standards.

Sudanese civilians affected by war have shown a high prevalence rate of PTSD symptomology, ranging between 36\%-48\% (Karunakara et al., 2004; Peltzer, 1999; Roberts, Damunda, Lomoro, \& Sondrop, 2009). Comparable PTSD symptom rates have been recorded amongst Darfuri (IDPs) within camps in Darfur 45\%-75\% (Hamid \& Musa, 2010; Kim, Torbay, \& Lawry, 2007; Morgos, Worden, \& Gupta, 2008; Rasmussen \& Annan, 2009; Rasmussen et al., 2010; Souza, Yasuda, \& Cristofani, 2009).

Co-morbidity plays a role, where individuals with co-morbid PTSD and MDD have worse long-term outcomes than those with either depression or PTSD (Momartin, Silove, Manicaasagar, \& Steel, 2004). Some research has found higher rates of clinical disorders such as PTSD, MDD and dissociation (Kinzie et al., 1990) and greater psychological disturbance (Fazel \& Stein, 2003). In other studies, prevalence rates among waraffected civilians are even lower than those of the host population (Beiser \& Hou, 2001; Steel, Silove, Bird, McGorry, and Mohan, 1999; Steel, Silove, Phan, and Bauman, 2002). There has been considerable inconsistency in rates of PTSD reported, ranging from $7 \%$ to $86 \%$ according to some estimates (Fawazi et al., 1997).

With two-fold greater lifetime prevalence for women than men (Basoglu, Salcioglu, \& Livanou, 2002; Kessler, Sonnega, Bromet, Hughes, \& Nelson, 1995), psychiatric comorbid disorders such as major depression symptoms (Armenian, Morikawa, \& Melkonian, 2002; Kuo, Tang, \& Tsay, 2003) and generalized anxiety disorders (Good \& Kleinman, 1985) are likely due to a direct result of war trauma and war-related violence against women. The development of severe and persistent major depression symptoms (Carr, Freund, \& Somani, 1995) including a more diverse set of psychological and psychosomatic symptoms, such as social withdrawal, attention deficit, headaches, faintness, dizziness, chest pains, low energy levels, hopelessness, helplessness, guilt and worry are more likely to be experienced by war-traumatized women (Mollica, Poole, Son, \& Murray, 1997; Roth, Newman, Pelcovitz, van der Kolk, \& Mandel, 1997; Tedeschi \& Calhoun, 1995). Moreover, the psychological sequelae of sexual violence and abuse surrounding women per se have resulted in an increase in fear and anxiety levels (Whitfield, 1995), anxiety attacks (Krishnan et al., 2004), generalized anxiety disorder (Peltzer, 1999) among Sudanese women. More recently, Darfuri women have reported a loss of dignity, survival guilt, uncertainty, shame, loss, and a lack of normality, showing a high prevalence rate for generalized anxiety disorder (Kim, Torbay, \& Lawry, 2007; Morgos, Worden, \& Gupta, 2008). 


\subsection{Psychosocial impact of war-related and post-displacement stressors}

There is evidence that war-related traumas are associated with the impact of daily life stressors and hassles, adverse social conditions, lack of material resources, and substantial economic loss contributing to the overall psychosocial health and post-trauma functioning among war-traumatized populations (Palmieri, Galea, Canetti-Nisim, Johnson, \& Hobfoll, 2008). Advocates of the association between war-related and postdisplacement stressors draw on a wide range of psychosocial issues relating to postdisplacement difficulties, which may arise as a result of incompatibility to the host community. A strong cultural component can be determined by the disruption of community bonds, culture shock, assimilation, and social marginalization (Young, 1995), and the lack of social structural support systems (Berman, Giron, \& Marroquin, 2006). Living in close proximity, lack of privacy, the lack of basic needs posed by the extraordinary life experiences amongst IDPs, and the lack of normality, often shared collectively, emphasizes the role that daily stressors play in mediating direct war exposure and mental health outcomes within a cultural context (United Nations, 2005).

The experience of loss in resettlement has been linked to mental health outcomes among war-traumatized populations. Individuals who report a loss of meaningful social roles and loss of important life projects (Miller, 1999), report lower levels of daily activity (Miller, Weine, Ramic, Brkic, Djuric Bjedic, Smajkic, A., 2002), are facing economic hardship (Beiser \& Hou, 2001; Simich, Hamilton, \& Baya, 2006) and/or report being socially isolated (Miller, Weine, Ramic, Brkic, Djuric Bjedic, Smajkic, 2002; Mollica et al., 2001), are all at risk of worse outcome in the host community.

Several features are in effect which maintain the relation between war-related traumatic events and post-displacement mental health. Primarily significant is the doseresponse association where the severity of PTSD symptoms increases as war-affected civilians' exposure to traumatic experiences increases (Fawazi et al., 1997; Kinzie et al., 1990). This concept has been coupled with the ongoing separation from significant others and the uncertainties and stresses maintained by unfamiliar post-displacement stressors and daily hassles that challenge and limitation the available support systems in the host environment experienced by many war-traumatized civilians (Kaplan, 2009). Furthermore, different time frames have been suggested to explain the psychosocial fractures that occur to war-affected civilians whereby an increased level of distress and symptoms are evident in the early stages of resettlement, given the demands placed on resources, and then a subsequent decrease in symptoms when those initial stressors have passed (Beiser, 1988; Tran, Manalo, \& Nguyen, 2007).

Moreover, the importance of demographic variables for predicting psychosocial outcomes among war traumatised individuals in resettlement has suggested that war- 
affected civilians had worse outcomes than their non-war affected control comparisons. Factors that influence the psychosocial outcomes of resettled war-affected individuals were restricted economic opportunity, being internally displaced as opposed to refugees, coming from unresolved ongoing conflict areas, low SES, being female, and those who were from rural backgrounds (Majka \& Mullan, 1992; Porter \& Haslam, 2005).

\subsection{Coping strategies with war-related trauma and post- displacement stressors}

An array of theoretical frameworks have attempted to define the factors and circumstances of internally displaced populations; in effect describing their incidence and prevalence from a cross-cultural perspective (Betancourt, 2005; Paardekooper, De Jong, \& Hermanns, 1999), defining their emotional manifestations from a psychological and psychosocial perspective (Thabet, Abed, \& Vostanis, 2002; Thapa \& Hauff, 2005), and relating their ensuing psychological dysfunction and attempts to cope from a psychosocial perspective (Morgos, Worden, \& Gupta, 2008).

Though there are resemblances within the coping and adjustment literature to define, identify, recognize and explain coping strategies, variations and distinctions depend primarily on the models and approaches of various theorists such as the utilization of resources (Hobfoll, 1989), individual dispositions and personality traits (Lazarus \& Folkman, 1984), and social support (Sarason, Sarason, \& Pierce, 1994), the focal point where all theorists come together is to demonstrate a series of steps, thought processes and particular behavior patterns that people take when confronted by a stressful situation.

Coping is an interactive process deeply embedded in context. Variations in external conditions, appraisals and changing cognitive and behavioral efforts and skills may be perceived as particularly taxing when the demands of a threatening situation (Lazarus, 1993), such as forced displacement is presented on one's personal resources (Hobfoll, 1991). Accordingly, a population of young African displaced females, this may be perceived as particularly stressful to their personal resources and all their efforts are directed at regulating emotional stress and/or dealing with the problems at hand in order to manage the troubled person-environment transaction. Through a multi-disciplinary framework of psychosocial and socio-cultural theory, the present studies reported in this dissertation focus on describing the demographic background, including tribal affiliations, the circumstances from which they left; the severity of forced uprooting issues and traumatic war-related life events; post-displacement issues as a result of civil war; exploring their current mental and psychosocial health dysfunctions (PTSD symptomology) and the effective coping strategies and available social support systems of an internally displaced young Darfuri women currently living in Omdurman city. 


\subsection{Impact of ethnic minority background and low SES on female university student's adjustment}

University life in and of itself creates academic, social and even financial hurdles for many entering into tertiary education, which may be associated with psychosocial stressors and adjustment problems among undergraduate students (Vaz, Mbajiorgu, \& Acuda, 1998). The dilemma is even more pronounced as ethnic minority students are faced with and try to adjust to a host community culture filled with unfamiliar social norms and customs in the academic and social environments (Wei et al., 2007).

The psychosocial stressors for many undergraduates forced to live away from home and family such as those affected by war-related experiences, those coming from rural areas, and ethnic minority students may encounter difficulties, conflict, and stressors of daily life. Stress due to culture shock, differences in languages and linguistic dialects, and values have been shown to negatively impact on physical health problems (Paukert, Pettit, Perez, \& Walker, 2006), interpersonal social relationships (Duru \& Poyrazli, 2007), social adjustment (Sandhu \& Asrabadi, 1998), appetite and sleep disturbances, low energy and stamina levels, and headaches (Ye, 2005), and psychosomatic illnesses (Greenland \& Brown, 2005). Especially among minority undergraduate female student's aspects of culture shock due to change and differences in the socio-cultural environment has been related to perceived racial and social discrimination, perceived hate, hypersensitivity to perceived verbal and nonverbal behaviours of others, homesickness and loneliness due to separation from emotional and social support of significant others, and fear that comes from feelings of insecurity and unfamiliar surroundings (Sandhu \& Asrabadi, 1994).

SES is considered indicative of the level of resources; material and societal, which can be defined for an individual, or community (Gallo \& Matthews, 2003). The psychosocial impact of SES among undergraduates from low to medium SES backgrounds has indicated that they may be more prone to exhibit countless emotional sensitivity due to their unfamiliar surroundings (Dahlin, Joneborg, \& Runeson, 2005).

Ethnic minority status and low SES students have been identified as a risk factor, suggesting that it may be associated with negative outcomes in areas that include academic performance and psychological well-being (Becker et al., 1992; Moritsugu \& Sue, 1983) particularly anxiety and depression symptoms imposed by academic, language, financial, and even political hurdles (Wei et al., 2007). Furthermore, the onset of depressive episodes have been related to academic performance pressures, the lack of learning materials to them (Kelly, Ryan, Altman, \& Stelzner, 1993; Rice, Herman, \& Petersen, 1993; Seidman et al., 1995; Seidman, Allen, Aber, Mitchell, \& Feinman, 1994), unrealistic self-expectations and expectation from their significant 'others' (Moffat, 
McConnachie, Ross, \& Morrison, 2004; Sherina, Rampal, \& Kaneson, 2004a, 2004b) and test anxiety (Hamad, 1974; Sharma, Parnian, \& Spielberger, 1983).

Additional stressors of everyday life related to social problems, finance and budgeting, accommodation adjustments, and academic challenges have been shown to increase the risk of anxiety disorders and depressive episodes among university students (Bramness, Fixdal, \& Vaglum, 1991; Hysenbegasi, Hass, \& Rowland, 2005; Tomoda, Mori, Kimura, Takahashi, \& Kitamura, 2000) and the possibility of associated factors such as the occurrence of daily life stressors (Paardekooper, De Jong, \& Hermanns, 1999) have also predicted a higher than expected prevalence of anxiety and depression symptoms amongst urban poor populations, particularly amongst women students (Broadhead \& Abas, 1997; Lundberg, Cantor-Graae, Rukundo, Ashaba, \& Östergren, 2009).

\subsection{Coping strategies and resilience among female university students}

It is important to note that, although coping and resilience have been used interchangeably within the literature, they are different constructs - coping refers to the cognitive and behavioral strategies used to manage the negative effects of stressful events (Lazarus \& Folkman, 1984), whereas resilience illustrates a persistence to surpass challenges and thrive to produce a positive outcome in spite of stressful events (Snyder \& Dinoff, 1999). Studies have shown that active coping responses, such as seeking guidance and logical analysis, were significant predictors of the relationship between stress levels and adjustment (Folkman, 1997), plus a satisfaction with academic and social life (Ye, 2005) and of meaning in life (Pan, Wong, Chan, \& Joubert, 2008) among ethnic minority undergraduate students. Furthermore, planned action coping responses illustrate the importance of a problem-focused, active approach for positive psychological functioning where perceived barriers and cultural incongruity ultimately increases resilience during tertiary education (Gloria, Castellanos, \& Orozco, 2005). While passive coping responses, such as cognitive avoidance, acceptance, resignation, and emotional discharge predicted increased stress (Vergara, Smith, \& Keelea, 2010), decreased intercultural competence and diminished effectiveness in intercultural interactions (Torres \& Rollock, 2004b).

The coping literature seems to point to the importance of active or problemfocused coping, the need to form new relationships and social support from friendships as a strategy for coping and dealing with tertiary psychosocial stress and college adjustment among students (Mena, Padilla, \& Maldonado, 1987; Vazquez \& GarciaVazquez, 1995; Williams \& Berry, 1991). Furthermore, the quality and satisfaction with familial support and the encouragement plays a vital role in the prediction of congru- 
ency between academic pursuits, traditional values and gender role expectations among female university students (Zea, Jarama, \& Bianchi, 1995). Studies have shown that the quality of social support among female undergraduates is associated with feelings of guilt about spending time at the university instead of undertaking domestic duties, as well as the detrimental effects of university drop out, (Gloria, 1997; Haro, Rodriguez, \& Gonzales, 1994; Torres \& Rollock, 2004a; Young, 1992).

In an educational environment resilience refers to "students who despite economic, cultural, and social barriers still succeed at high levels" (Cabrera \& Padilla, 2004). The relationship between resilience and coping styles, especially among female undergraduates showed that both task- and emotion-oriented coping were significant predictors of resilience (Campbell-Sills, Cohan, \& Stein, 2006) . Resilience among undergraduate students has been conceptualized to reflect that even though students may initially experience challenges in academics and social relations as a result of cultural differences, individuals ultimately thrive and become socially adjusted and academically successful (Ogbu, 1992). Furthermore, its relevance has been illustrated in tertiary adaptation especially with respect to areas of high self-esteem (Phinney \& Alipura, 1990), academic achievement (Hackett, Betz, Casas, \& Rocha-Singh, 1992), and positive social interactions with others (Holleran \& Waller, 2003). The levels of resilience coupled with the frequency and quality of social support defines undergraduates' experience of stress, culture shock, perceived discrimination, and homesickness particularly amongst ethnic minority female students' (Thomas \& Choi, 2006). Furthermore, social adaptation perspectives have suggested that adhering to traditional values and belief systems are a source of strength that promote resilience in the face of obstacles where culture serves as a protective reservoir of coping and adaptation strategies (Saleebey, 1997).

\subsection{Exploring mental health and psychosocial interventions in the Sudan}

\section{Mental health in the Sudan}

Within its current state of contextual disarray, the Sudan, is now gaining a reputation as being the most famine-stricken, conflict-ridden, and war-plagued nation in the continent, and is among the top ten percent of poorest countries in the world, with spiralling neglect of natural and human resources, lack of infrastructure, inadequate facilities, limited accessibility of services and poor governance. Spending $41 \%$ of the GDP on defence it is no wonder that there are endemic shortages on educational ( $8 \%$ of the GDP), and health sectors including mental health facilities (3.8\% of the GDP) (World Bank, 2005). Furthermore, with only 4 psychiatrists, and 4 psychiatric beds, 2 psychiatric nurses, 5 psychologists and 9 social workers per 10,000 in the capital, Khartoum, the lack of ser- 
vices and professionally trained mental health care providers significantly challenge the increasing prevalence of mental disorders (World Health Organisation, 1992).

In relation to IDPs mental and psychosocial health PTSD has often been highlighted as the main outcome of war related traumatic experiences (De Jong, 2002; Drozdek, 2003). High co-morbidity levels of physical symptoms and complaints ratings have suggested an underlying emotional component and a sign of somatization, as well as often elucidating psychotic dysfunction including schizophrenia, hallucinations, major depression disorder and generalized anxiety disorder among IDPs who have witnessed trauma (Geltman et al., 2005).

Although accounting for approximately $70 \%$ of war exposed groups (Sapir, 1993), IDP women have traditionally been marginalized in the study of mental health care provision without redress to effective psychosocial and mental health care provision which highlights their potential for developing future dysfunctional psychosocial capacities (Foa, Keane, \& Friedman, 2000). For women, post-displacement difficulties of daily stressors were similarly predictive of all mental health outcomes and functional impairment as were war-related experiences of violence (Miller, Omidian, Rusmussen, Yaqubi, \& Daudzai, 2008), highlighting the importance of cultural compatibility with the host culture (Silove, 1999). The literature has identified the psychosocial impact of postdisplacement life stressors amongst war-traumatized women, including extensive suffering and trauma, injustices, a lack of security, fear, shame, lack of trust, and loss of community ties, identity-role, and existential meaning (Igreja, Kleijn, \& Richters, 2006). Furthermore, published works that relate to the psychosocial implications of war exposures on Darfuri women and girls illustrated that they were exposed to crimes of gender based violence the use of repression and oppression in Darfuri IDP camps (Morgos, Worden, \& Gupta, 2008).

In an attempt to address the mental and psychosocial health of IDPs in Darfur and Khartoum based camps, humanitarian groups such as UN agencies, international nongovernmental organizations (INGOs), and Sudanese government led Humanitarian Aid Community (HAC) have put together health promotion intervention initiatives and plans of action. These included assessing health needs including mental and psychosocial health, professional capacities, and facilitating public-health including community participation (OCHA, 1999). However the lack of a coordinated effort to include the support of local psychiatric and psychosocial mental health promoters has lost much of the needed momentum from these social networks in the sustenance and development of the interventions (OCHA, 2006).

Despite high rates of psychosocial impairment among war-affected Sudanese (Coker, 2004a; Nilsson, 2000; United Nations High Commissioner for Refugees, 2000) the psychological rehabilitation efforts has seen many challenges. 
Intervention programs that are formulated for promoting mental health and psychosocial rehabilitation among war-affected displaced populations have to be prepared to offer services that are culturally and ethnically viable. In order to offer culturally competent services, mental health professionals are required to be familiar with every component of the community they aim to help such as demographics, gender, the traumas encountered as a result of the civil war, factors leading to massive exodus, and the difficulties of the transit and post-displacement stressors, and the possible acculturation stress that is manifested in the form of integration with the host community.

\section{Theoretical Conceptual Framework}

Operationalizing an effective intervention program that reflects the totality of experiences among female war-affected IDP undergraduates must incorporate concepts from theory in a dynamic interactive process that mirrors an IDPs' personal road to recovery.

Concepts that may be implicated in describing the impact of war-trauma and displacement stressors on ethnic minority female students are the theory of meaningrelated processes (Folkman, 1997; Folkman, Moskowitz, Ozer, \& Park, 1997); resilience (Masten, Best, \& Garmezy, 1990; Rutter, 1985); and positive protective social support networks (Higgins, 1994; Werner, 1990).

The theory of meaning-related processes is tightly intertwined within culture, tradition and religiosity in that it provides a way of understanding unchangeable negative events (Wortman \& Silver, 1992). The process involves reappraising events that are usually beyond the control of the person. Attributing new meaning to the events and subsequent emotions that arise typifies a coping strategy that is used in harsh experiences such as war-related traumatic exposures and post-displacement stressors and the consequent feelings of suffering and loss (Lazarus \& Folkman, 1984). Furthermore, meaningfulness of life (Wagnild, 2008) has been used to describe the extent to which reappraised new situations help war-traumatized individuals deal with and offset the negative perceptions of war-related traumatic exposures and post-displacement stressors permitting realistic coping and a positive outcome in their adjustment process in the form of growth (Lerner \& Gignac, 1992).

Resilience connotes inner strength, competence, optimism, flexibility, and the ability to cope effectively when faced with adversity (Johnson et al., 2009; Rutter, 1985). Resilience is operationalized by cultural norms and societal expectations (Luthar, Cicchetti, \& Becker, 2000), manifested in traditionally sanctioned productive roles and responsibilities which develops and fosters a positive self-image (Bronfenbrenner, 1979), social confidence (Garmezy \& Tellegen, 1984), prosocial behaviour (Macksoud \& Aber, 1996), and self-efficacy (Ferren, 1999; Maslow, 1943).

Culture therefore seems to play a huge role in resilience formation among individuals who are mobilized by internal motivation to eliminate stressful conditions, reconcile 
adverse experiences and achieve psychosocial adjustment in the face of adversity (Masten, Best, \& Garmezy, 1990). As such ethnic minority students may possess the internal attributes of specific cultural norms (Luthar, Cicchetti, \& Becker, 2000; Luthar \& Cicchetti, 2000) that enable them to meet defined societal and cultural expectations on the basis of behavioural competence (Elder, 1998), generating opportunities for success within their immediate environments (Scarr \& McCartney, 1983; Werner \& Smith, 1992), positive self-perceptions and external competences (Luthar, 1991), environmental mastery, and academic achievements (Greenberg, Lengua, Coie, \& Pinderhughes, 1999).

Positive social supportive networks protect and buffer against adversity and severe distress by providing active reassurance, guidance, belonging, and affection in the availability, range, frequency, accessibility and quality of close relationships and attachments IDPs make in terms of alleviating psychosocial disorders (Henderson, Byrne, \& DuncanJones, 1981; Rutter, 1981; Schaefer, Coyne, \& Lazarus, 1981; Shapiro, 1995).

This interactive theoretical framework helps to explain the effective ways by which important concepts need to be considered when planning for a culturally appropriate and targeted war-trauma training program for Sudanese trauma counselor.

In seeking to identify the psychosocial and mental health needs of Ahfad University for Women (AUW) war-affected undergraduates', the university set out to develop an in-house psychosocial and mental health service as part and parcel of university infrastructure for the relief or control of the progression of mental health illness among its IDPs.

\subsection{Dissertation Outline}

A systematic approach was used to reach the aim of this dissertation which was to identify the mental health and psychosocial needs of Darfuri IDP war-affected undergraduate women at AUW; the effects of their current post-displacement stressors and daily life hassles; their psychological dysfunctions (PTSD, generalized anxiety and depression symptomology); and available and effective coping strategies, coping resources and resilience characteristics.

The dissertation offers an integrative multi-disciplinary framework of psychosocial and socio-cultural concepts to capture the multi-factorial dimensions that have existed within the lives of female internally displaced AUW undergraduates. Moving from an exploratory oriented research towards an action-oriented research, the dissertation amalgamates evidence and theory based information to outline the objectives for a sustainable Sudanese war-trauma counselor training program whilst generating knowledge about and understanding of war trauma experiences of women in general, how such experiences are interwoven with daily hassles after displacement, and how they 
cope with their trauma's and daily hassles. As a consequence, Chapters 1, 2 and 3 describe explorative research regarding the mental and psychosocial impact of experiences of and exposures to war-related traumatic events and post-displacement stressors. This explorative research covers the literature in the field of mental health among war-affected populations, and describes personal accounts of the sample as well as the impact in terms of PTSD. Subsequently, in Chapters 4 and 5, the mental health status of war-affected and non-war affected civilians are compared, taking into consideration factors such as SES and ethnic minority.

Chapter 2 (Badri, Crutzen, \& Van den Borne, 2012a) presents the personal accounts of war-related exposures, current on-going life challenges, emotional distresses, and coping strategies amongst a sample of Darfuri female undergraduates studying at AUW. Chapter 3 (Badri, Crutzen, \& Van den Borne, 2012b) investigates the association between war-related exposures and assesses post-traumatic stress disorder (PTSD) symptoms amongst a sample of Darfuri female university students at Ahfad University for Women (AUW) in Omdurman city. Chapter 4 (Badri, Crutzen, \& Van den Borne, submitted) investigates and compares the mental health status in terms of anxiety, depression and resilience between war-affected Darfuri and non-war-affected Omdurmani undergraduate students at Ahfad University for Women in Omdurman city, Sudan. Chapter 5 (Badri, Crutzen, \& Van den Borne, submitted) explores and compares external coping resources and internal resilience characteristics among two ethnogeographically different Sudanese female undergraduates. War-traumatized, internally displaced, rural Darfuri students were compared with Omdurmani non-war traumatised, urban host students to investigate differences in effective coping to daily stressors in term of academic, social and financial matters. Chapter 6 (Badri, Crutzen, Van den Borne, \& Eltayeb, submitted), provides evidence- and theory- based guidelines for developing a war-trauma counsellor training program in the Sudan, mainly based on qualitative and quantitative studies among war-affected Darfuri female students. Cultural conceptualizations such as gender roles and religious expectations as well as theories that emphasize resilience and other psychosocial adaptation skills have been operationalized to reflect the totality of the Darfuri women's experiences. In Chapter $\mathbf{7}$ the results of the presented studies are discussed and integrated in a general discussion, which also offers implications for practice and future research. 



\section{Chapter 2}

\section{Experiences and psychosocial adjustment of Darfuri female students affected by war: An exploratory study}

"There is no greater agony than bearing an untold story inside you."

Maya Angelou

This chapter has been published as Badri, A., Crutzen, R., and Van den Borne, H.W. (2012). Experiences and psychosocial adjustment of Darfuri female students affected by war: An exploratory study. International Journal of Psychology. 


\begin{abstract}
This paper presents the personal accounts of Darfuri students studying at Ahfad University for Women in Omdurman, Sudan. Their war-related exposures, current on-going life challenges, emotional distresses, and coping strategies were explored using a semistructured interview protocol amongst a sample of 20 students. Through interpretative phenomenological analysis (IPA), the Darfuri students' stories illustrated that they were exposed to an array of traumatic war events, were confronted with myriad current life hassles and urban-cultural challenges, and had high levels of emotional distress as they relocated to Omdurman city. Strong religious practices and beliefs (praying and reading the Quran), ability to form interpersonal relationships, availability of social support networks, and a positive future outlook seemed to lend to their ability to cope with their subsequent emotional distress owing to war-related exposures, current on-going life hassles and urban-cultural challenges.
\end{abstract}




\section{Introduction}

Throughout the 1980s environmental decline initiated by drought and desertification led to loss of livelihoods and livestock, food insecurity and famine in Sudan's western province of Darfur (Elnur, 1994). The resulting political and economic challenges led to ethnic tensions and civil war. As the war has ebbed and flowed, it is acknowledged that presently 2.5 million Darfuri are officially displaced (World Health Organisation, 2008).

Despite the on-going conflict in Darfur, recent research that has been conducted is within IDP camps in Darfur. Pre and post-displacement conditions have been investigated and have suggested that women and girls exhibit high incidences of traumatic reactions, mental and psychosocial dysfunction (Kim, Torbay, \& Lawry, 2007; Morgos, Worden, \& Gupta, 2008). Rasmussen and colleagues $(2009 ;$, 2010) suggested that psychosocial maladjustment may be a consequence of an interplay between the impact of war-related exposures and current on-going life stressors. Furthermore, other post displacement stressors may include cultural dislocation (Eisenbruch, 1991), loss of normative social support networks and poor accommodation (Porter \& Haslam, 2005).

The ability to integrate many different coping strategies to protect and buffer against emotional distress has been shown with other Sudanese populations affected by war. The use of religious practices, forming meaningful interpersonal relationships, and showing signs of determination, perseverance and resilience has been evidenced (Goodman, 2004; Khawaja, White, Schweitzer, \& Greenslade, 2008; Schweitzer, Melville, Steel, \& Lacherez, 2006).

\section{Methods}

\section{Study Design}

This study was undertaken as part of a larger on-going mixed methods design to investigate the psychosocial adjustment amongst female WAS. A prompt-guided interview protocol was developed to explore the narrative accounts of twenty AUW students by recounting their stories of war trauma and emotional distress reactions, their present day on-going challenges, hassles, and stressors, and their habitual coping strategies to identify the aspects that support their ability for psychosocial wellness. While an ethnosocio-demographic questionnaire was designed to establish the sample's background.

\section{Study Setting}

AUW in Omdurman, Sudan, is a seven thousand strong women's only campus for higher education. It is guided by its educational ethos to provide graduates trained within the community and serve as effective change agents. As such it draws students from all 
parts of Sudan, including the displaced from Darfur. The Darfuri student population at the time of study was approximately $10 \%$ of the total AUW student body. Study Setting

AUW in Omdurman, Sudan, is a seven thousand strong women's only campus for higher education. It is guided by its educational ethos to provide graduates trained within the community and serve as effective change agents. As such it draws students from all parts of Sudan, including the displaced from Darfur. The Darfuri student population at the time of study was approximately $10 \%$ of the total AUW student body.

\section{Sample Selection}

Subsequent to obtaining ethical approval from the Ethics Committee of AUW, the registrar office was contacted to provide an updated list of registered Darfuri students. Two Darfuri students' union leaders helped with the recruitment of participants using a networking methodology. By word of mouth and distribution of flyers a total of seventyseven potential candidates registered as willing participants. Using purposive sampling, the selection process from these invitees was based on adhering to the following inclusion criteria: extended family must be of Darfuri origin, born and raised; parents, siblings and extended family must reside in Darfur; she had been in Darfur up to at least 2005 (war broke out in 2003; massive destruction and displacement followed; (United Nations, 2005); and her first visit to Omdurman city was to continue her education. Students who fit these criteria were then randomly selected from the pool of accepting participants. For the purposes of this study twenty students were invited to participate. Following written consent from each of the 20 Darfuri students, the principal researcher gave concise information about the interview procedures, especially the use of a tape recorder. None of the participants refused the use of the recorder and no tape was damaged nor discredited. Each interview was tape recorded within AUW campuses during university hours in December 2008. Conducted by the principal researcher each interview took approximately two hours. Owing to the sensitivity of the topics discussed and the possibility of students becoming distressed, referral procedures were put in place by collaborating with two in-house counsellors.

\section{Participants}

Broadly speaking the participants represented all main regions and ethnicities of the Darfuri AUW student body, studied within one of the six university faculties, and ranged in age between 17-22 years. Two main ethnicities divide western Sudan: African and Arab tribes. The majority $(\mathrm{n}=14)$ of the participants were represented by the Fur, Zaghawa, Massaleet and Bargu African tribes of northern and western parts of Darfur, and six from the Rizaigat Arab tribes of southern Darfur. Seventeen students have previously been displaced with their family from their villages on the outskirts of AlGinaena, Nyala, and ElFashir towns; their families are currently living in IDP camps. 
The remaining three participants have not been displaced as their place of origin is the town of AlGinaena. The occupations for most of the Darfuri sample's parents were herders and farmers; owning their land, the crops they grow and the cattle and camels they shepherd for income and subsistence, however these livelihoods were lost as a direct result of their displacement.

\section{Instruments}

A self-administered 17 short-response questionnaire was designed to establish the ethno-socio-demography of the Darfuri students. The students were asked about their geographical origin, (name of village and location to nearest town) duration since they left their place of origin, and means of travel to Omdurman; their tribe, age, birth order, and university level; parental economic status and occupation, parental property ownership, location of property, type of property and property loss; and present address.

To explore war-related exposures, current on-going life hassles, challenges and stressors, emotional distress levels and habitual use of coping strategies a promptguided interview protocol was designed using prompts only to initiate conversation and encourage the flow of participants lived experiences in both settings; Darfur and Omdurman. Ample time was given to establish trust and rapport allowing students to feel at ease in their disclosures and the freedom to express their emotions and retell their stories in their own words (Ghorashi, 2008). War-related exposure prompts asked for specifity, such as, prescribed periods of time, exact locations, and particular incidence(s). Participants were encouraged to elaborate on whether they were directly affected by, witness to, or heard about a particular incident and how these experiences have directly affected them emotionally.

Prompts related to on-going life hassles, challenges and stressors typically probed into the current lives lived within their 'host' environment, whether in the city or at the university. Specific hardships, stressors, and types of hassles were retold with special emphasis on emotional reactions. Typically a dialogue ensued of a characteristic day within their 'host' environment and how specific incidences made them feel.

The final section of the interview focused on discovering their psychosocial adjustment and to explore the ways in which war experiences and on-going life hassles and challenges exert their influences on habitual coping strategies, styles and modes of behaviours as a means to manage stress. To guide the discussions prompts focused on social support networks, environmental mastery, and future wishes and aspirations.

Both instruments were designed in English and translated to Arabic by the principal researcher. The Arabic interpretation was cross-checked and back-translated by two professional translators for cultural appropriateness and original semantic meaning. 


\section{Analysis}

To determine the ethno-socio-demographic composition of the participants, individual questionnaires were grouped manually according to ethnicity (tribal and sub-tribal), socio-economic status, and demographic background.

The original recorded word and initial transcription was in Arabic. Each transcribed manuscript was then carefully translated into English. Due to language disparities and to ensure linguistic reliability and validity and to make the stories flow more easily in English, syntactical and grammatical alterations had to take place within the English translation. Twenty-five per cent of the English transcripts were subsequently randomly selected then back-translated by two professional linguists confirming translation accuracy.

In preparation for coding and consistent with the guidelines for interpretative phenomenological analysis (Smith, Jarman, \& Osborn, 1999), the raw data was read and reread in its entirety several times to identify recurrent salient and shared experiences. Three recurrent core categories emerged: war exposures, current on-going life hassles, and coping strategies. Reapplying these core categories to the data as a whole made it possible to make connections and identify a preliminary set of codes. By looking for patterns and frequency of expressions to each successive transcript six themes were then classified and noted in list form for each core category: (a) personal and family war exposures, (b) witnessing warfare, (c) hearing about incidences within combat zones, (d) current life hassles and family's physical distance, (e) urban-culture and academic challenges (f) patterns of coping strategies. To capture the essential qualities of each theme significant quotations to support it were produced. Inter-rater reliability of data extraction was performed by two other researchers in the field to ensure that the emergent themes could be traced to the original quotation in the transcribed narratives (Mays \& Pope, 1995).

Emotional distress reactions were coded by forming two distinct lists: directly mentioned by the participant; and observations of emotional manifestation during the interview. These two lists branched further into 'war-related emotions' and 'on-going life hassles'. Patterns emerged forming thirteen recurrent distress reactions to warrelated trauma and seven distress reactions relating to current life stressors. Furthermore many of the war-related emotional distress reactions may be similar to symptoms of mood and anxiety disorders. For example, extreme sorrow, grief, mourning, and bouts of crying are identical to the DSM IV criteria for major depression. While, constant worry, excessive guilt, panic and fear are symptoms of anxiety disorder. 


\section{Results}

\section{Personal and family war exposure}

Recurrent themes of war-related confrontations on a personal level were rife. Experiences within combat zone battles of heavy gun-fire, artillery attacks, and air raids, separation and loss, injury, torture and death, kidnap, abduction and disappearance were retold in abundance. Prominent feelings of extreme sorrow, constant worry, grief and excessive guilt fill each story. Seventeen Darfuri students sorrowfully and with a deep sense of loss, describe how combat situations forced them and their families to flee their villages and homes to find shelter and escaping to save their lives. A Fur retells her story:

It was in 2003 when we had to escape, in fear for our lives, we could not endure the constant shelling and sounds of the helicopters above. We had no shelter, no property, and now we lived in camps packed with others who had come before us. The most terrifying times were those when I saw children so small and the elderly so frail not find-

ing a safe place to stay, confused and in fear with no food to eat nor water to drink.

All twenty Darfuri participants grieved for the death or critical injury of more than one family member. Whether receiving beatings or bullet wounds to the head, torso or limbs during violent interrogation, four students lost their father, three lost their brother, three lost their uncle, and one lost her sister, seven lost a cousin or relative and one lost her mother.

Kidnapping, disappearance, and abduction of brothers, sisters, cousins, grandfathers and other family members was yet another event that brought constant worry and apprehension to most Fur participants. As one of them explains:

My mother, sisters and me were attacked during broad daylight as we fled our home ... seeking refuge and sanctuary .... We were ambushed and kidnapped by men who carried different types of weapons and guns. We spent four days in captivity with them. And every day they aimed their guns at us. I felt petrified that they might harm us, but they did not. After four days of living in desperation they set us free...and...we continued our track safely...

Loss of property by way of burning, looting or destroying farms, crops, and livestock, was also typically mentioned. Confiscation of property deeds or forcibly revoking land ownership rights, and reinstating possessions to different tribes was mentioned by most of the Massaleet and Bargu tribes. A Bargu participant explains:

... our homes and property [were] attacked... Our area was completely destroyed; our plantations and livestock were destroyed; we lived in constant fear and terror. We sought out the protection of the police, but they too would ambush, loot and steal our 
belongings. We could not salvage our property and belongings from the gunmen, nothing was left!

\section{Witnessing warfare}

Although each narrative is unique all the Darfuri participants have witnessed the violence that occurred 'right in front of my eyes', events like 'homes being burnt and destroyed, people being robbed by bandits', and seeing the 'wounded and amputees, orphans and homeless children filling our roadsides' brought back feelings of extreme sadness, guilt, terror, and shame.

\section{Hearing about incidences within combat zones}

Darfuri students have had news relayed back to them through family, relatives and friends since their arrival to Omdurman. The most recurrent topics shared by the group was shortages of essential supplies like food, clean water and medicines; parents and family members becoming weak; sick and diseased; being left behind and without shelter. Emotions which marked these narratives were mainly despair, grief, bitterness, and worry.

This emotional 'baggage' which sits squarely on these participants shoulders is inevitably carried along as they embark on their journey and attempt to resettle in $\mathrm{Om}$ durman as university students.

Current on-going life hassles presents the second core category provided by the sample's narratives. At the time of study, twelve Darfuris had been in Omdurman city for less than a year, while the remaining ranged between 2-3 years. Living in various university hostels their experiences reflected two recurrent themes: a. current life hassles and family's physical distance, and b. urban-culture challenges and lack of environmental mastery

\section{Current life hassles and family's physical distance}

Family is an important part of the Darfuri community structure and function, which usually extends beyond nuclear boundaries to include aunts, uncles, cousins and grandparents. The present sample of Darfuri students reported finding it "extremely" difficult being far away from parents and family members; they experienced "profound family disruptions", and an "uncertainty as to the whereabouts of family members in Darfur". This may be because: "News is scarce and sometimes unconnected; it is sometimes difficult to know where they are now. I pray to Allah that they are well and safe." A certain amount of loneliness, were mentioned as they described a sense of loss in family and community ties. A Fur recalled: "I am missing having my sisters and brothers around me; we are a family of twelve. Waking up together chatting, joking, laughing and playing... 
smelling the sweet scent of my mother's delicious porridge out there on the fire. I feel very lonely over here at times".

A substantial number of Darfuri participants $(n=15)$ said they mourned the loss of rich and familiar social networks to which they belonged and would turn to for assistance and support, as mentioned by a Bargu student: "I miss having my family around me in times of need. Even though I am surrounded by others like friends over here ... there is always an emptiness that cannot be easily filled". Many were used to having the shelter of parents and the encouragement of other extended family members like elder cousins, who served as role models and who set up boundaries and appropriate codes of conduct, customs, dress codes and behavior.

Furthermore, lack of parental guidance and continuous supervision was of great concern especially for a large number of newly-arrived students ( $n=14)$, who spoke of a new found freedom came with: "too many choices... and too much freedom". But also with the lack of parental supervision they realized that more self-reliance was created: "I am mostly on my own over here. It is always a financial struggle for me nowadays, but I must depend on myself and show that I can be trusted by my family".

\section{Urban-cultural and academic challenges}

All participants have come to Omdurman directly from Darfur; this is their first experience of being on their own. Most Darfuri students mentioned situations such as negotiating the transport systems and learning the routes and directions to important city landmarks as "challenging". Also as prices of basic essentials (hygienic products, lunch money, bus fares, clothes and library card fees) are very high, insufficient funds has led to most students accepting hand-outs or go without for a time each month; this some say is 'very humiliating' and 'worrying'.

Without exception, Darfuri students at AUW are provided with an educational scholarship. Most use this towards renting space in a room in one of the seven exclusively shared Darfuri self-catering hostels. For the first time they are experiencing living on their own, albeit, in uncomfortable, overcrowded living conditions with 120 students to a hostel of eight rooms and four bathrooms. Consequently, they described having experienced a dramatic change in life style; having no privacy, lack of hygienic living space, and loss of important documents, assignments, and references. A Massaleet sophomore retells her experience: "We cook, eat, sleep and study in this one room; life is too cramped".

Typically newly arrived Fur participants reported a significant cultural gap between their habitual norms with those of the dominant Omdurmani society. The broadening of social support networks as opposed to the more familiar closely knit widebased family structure seemed to render them somewhat incongruous with their new environments. Despite being Sudanese most Darfuri participants mentioned being ex- 
posed to unfamiliar norms, customs and even idiomatic language, made them feel estranged and uncomfortable, as was stated by a Rizaigat newcomer: "It is almost like being foreign in our own country: they dress less conservative; much too tight, I don't see myself as being similar to most girls I see. They speak in words and phrases that are unfamiliar to me and sometimes I get lost in conversations". Feeling uncomfortable and estranged to particular values such as a general care-free attitude towards life and freedom of movement was at times unnerving. A Rizaigat freshman reported: "I think we are a lot more conservative back home. I feel very uncomfortable".

Furthermore, a few participants mentioned that they have experienced subtle discriminative actions and stereotyping by the 'host' society, especially when speaking tribal dialects in public. Often rendering them embarrassed or ridiculed, one Fur student mentioned that her daily struggle with stereotyping and automatic discriminatory association was always an uphill struggle and challenge: "My homeland is being stripped barren, I am one who has lost everything. And yet I am ridiculed and feel ethnic tension and discrimination!"

University life also posed as daunting and overwhelming as almost all students mentioned some sort of academic concern. Lack of appropriate university study skills, computer and internet knowledge, library orientation, time management, staff expectations, and English language proficiency when writing assignments or understanding lectures caused serious anxiety and worry, as was mentioned by one Bargu junior: "Everything is in English; mine is very limited. So writing assignments, using the library and reading references are always a struggle. It's like when I first came here, trying to work on the computer was a terrifying experience. Switching it on and off was a challenge!" Ten freshman students in particular reported feeling frustrated as they attempted to access essential university information such as becoming involved in extra curricula activities and performances, which some found; "completely confusing!".

Coping strategies and patterns of coping behaviors represent the third and final core category extracted from the participant's narratives. Darfuri students identified several strategies, resources and responses which allowed them to cope with their stressors and improve their psychosocial adjustment. These coping responses included: a. reliance on prayer and religious belief; b. social support networks and interpersonal relationships; c. improving environmental mastery and intercultural competence; and d. focusing on future wishes and aspirations.

\section{Reliance on religious practices and beliefs}

All participants identified the use of religious practices as a coping strategy. Their faith and use of prayer seems to enhance hope for the future and facilitated their coping when dealing with each new challenge. They seemed resolved and mentioned that they 
would persevere rather than give up despite the on-going nature of hassles and hardships they encountered: "I have faith that God will provide me with enough power to cope I believe that God will provide me with the will so I must never give up". In times of difficulty they would pray to have the strength to continue, or pray for an improvement of their current situation, as a Bargu participant reported: "I turn to Allah for his mercy and give me the strength to continue. I ask Him to improve our situation". They would pray for the relief of hardships for their parents and families in their current plight and sorrows in Darfur, they prayed for a better future for themselves and a better future for their loved ones in Darfur. They also placed their fate in God and believed that whatever happened would be at the will of God. Another Bargu noted: "When you believe and put everything in God's hands, it happens". Participants also noted that they used their belief in God as a form of emotional support. Specially they described how praying to God provided them with relief and a sense of inner peace when they are unhappy or lonely as one Fur student reported: "I felt alone and lonely, life was getting very difficult, so I placed my load on the Almighty, and prayed for relief. I always do when I feel burdened". Also the use of prayer as a way of attaining hope for the future, especially during their first few months in Omdurman and as university students; "praying to Allah always helped me gain strength and confidence during my first months in Ahfad".

\section{Social support networks and interpersonal relationships}

All Darfuri students did not have any family members in Omdurman and were living without their usual familial networks, residing in hostels or dorms. Commonly, they reported making close interpersonal relationships with other Darfuri students, especially those of the same ethnicity, receiving emotional support particularly during times of difficulty and distress such as receiving upsetting news from Darfur. The social support networks now established covered a more broadly based range of individuals such as friends from the university or acquaintances from the Darfuri student union and residential neighborhood.

Most of the younger participants found themselves in a novel situation and mentioned that they would "spend more than six hours of each day speaking and sharing personal past experiences", "off-loading feelings about family" issues, "share thoughts and emotions with those who have similar experiences of loss" and homesickness. Older students were the providers of this emotional support and saw themselves as "role models". They also expressed a sense of obligation to build relationships with their younger counter-parts in an attempt to assist, advise and guide them on customary behaviors, acceptable norms, and exchange relevant information pertaining to their new environment, as one sophomore Massaleet student states: "of course if one of my sisters is asking for help, and sometimes even when she does not, I am there for her. We are all far away from our lands and all must be there for each other." 


\section{Urban-culture and academic competence}

Becoming familiar with street names, traffic signals, routes and transportation systems was seen as challenging but pivotal to achieving environmental mastery: "Routes can be very confusing and complex with so many buses and stops to keep in mind I had to always count the number of traffic lights it took from the hostel to the main market". Accepting the existence of cultural differences, learning new codes of conduct, building friendships with other tribes and sects from the community, learning new language eccentricities and affirming attitude changes within themselves made adaptation easier into their new urban-culture. Finding and accessing the right resources and information within AUW was viewed as paramount in helping them cope with university life: "If only I knew there were bulletin boards with valuable information, or that asking student union members to find resources like the in-house counseling program, life would have been a lot easier in the beginning".

\section{Focusing on future wishes and aspirations}

The most common wish reiterated by the majority of the participants was a determination to successfully complete their education at AUW. Becoming actively involved in pursuing their education facilitated their ability to focus on the future and enhance their coping in spite of on-going hassles: "My situation here is difficult. My English is weak and living quarters are extremely crowded. I am faced daily with new challenges. I am in constant fear for the lives of my family. But I cannot say to myself 'ahh just give up'. I know I am better off than many sisters back in the village; at least here I am safe. So I must keep going. I must stay and do the best I can. I must persevere". They demonstrated enormous purpose in continuing and completing their university education as they felt their return with new acquired skills would assist in building their communities: "My dream is to finish my university education successfully, so I could go back to Darfur, work for my people and improve their situation". Furthermore, the majority maintained hope for the future by comparing themselves with others who were less fortunate than themselves while minimizing their current difficulties and stressors, as one Fur participant stated: "I know sometimes money is tight, people are harassed and assaulted, but at least this is not Taweelah. I know many who are still going through more hell than me. I know I am fortunate enough because I'm in Omdurman, far away". This comparison with others allowed them to gain a more positive perspective and remind them that they had survived through worse experiences. With visions of a war-free and peaceful Darfur these students were nourished by a determination to preserve in their university education as a Zaghawa student said: "I share in the hope for peace and stability; suffering will be no longer and the killings will end. We must get back to starting new developments for our people so we can improve their quality of life and improve our future. This will make the beginning of a safe, calm and better life for all Sudanese." 


\section{Discussion}

While not currently living their family's hardships within IDP camps in Darfur, the baggage of being affected by war still remains etched deeply in their memories and within their psychological being. Theirs is a situation of surviving within conflict zones, becoming displaced within IDP camps and temporarily uprooted pursuant of educational opportunity within a culturally unfamiliar environment; Omdurman city and AUW.

This study contributes to the hallmark of personal war exposures experienced by other Sudanese populations (Paardekooper, De Jong, \& Hermanns, 1999). Three years on, our participants can vividly recall and retell their experiences of war exposure with descriptions of fleeing, loss, and death. Not only do these experiences reflect other Sudanese studies but also indicate the psychological impact that is left behind (Paardekooper, De Jong, \& Hermanns, 1999; Peltzer, 1999). The extent to which these Darfuri students still struggle with the emotional burden is exhibited by the description of their emotions: extreme sorrow, sadness, constant worry and excessive guilt. These emotions are similar to symptoms of major depression and anxiety signs of traumatic. Long term mental health repercussions of Sudanese affected by war have been discussed in the literature (Karunakara et al., 2004; Roberts, Damunda, Lomoro, \& Sondrop, 2010).

For the most part, the majority of our sample, particularly the newly-arrived undergraduate Darfuri, relied on a combination of religious practices and beliefs, such as prayer and faith to protect against emotional distress, specifically when they hear disturbing news from Darfur. By forming ethnically prescribed social support networks buffering against external odds, especially when faced with educational challenges and societal disapproval. And finally they consider completing their educational sojourn an enormous achievement for themselves, their families and communities as they anticipate a brighter future, which compels and motivates them to succeed despite financial constraints and even poverty at times. They have also mentioned repeatedly that they are determined and do preserver to keep on going despite the odds. The significance of effective coping strategies and endurance amongst other Sudanese affected by war is evidenced in the growing body of literature (Meffert \& Marmar, 2009; Stoll \& Johnson, 2007; Tipping, Bretherton, \& Kaplan, 2007).

Prior to this current educational journey, our sample of Darfuri female undergraduates have never travelled alone; lived alone; never handled money; never lacked parental guidance or supervision; never felt ambiguous nor uncertain about customary roles and habitual codes of conduct. As they embark to absorb the urban-cultural tapestry of Omdurman city and life as un-chaperoned females, they face a set of profound psychosocial challenges and stressors. Accepting cultural differences and relearning new acceptable codes of conduct made transition and coping with change a lot easier. 
Although being survivors of relatively short-term but extremely traumatic war exposures and compounded by their current on-going academic challenges and stressors the data does not suggest that the present sample may be at increased vulnerability for engaging in unhealthy academic behaviours such as having attention, memory and information processing problems that have been suggested by other Sudanese affected by war studies (Betancourt, 2005).

The communal attribute of collective coping that is shared by the bulk of our Darfuri participants may reflect that they see themselves predominantly as being part of a group with shared experiences that were previously characteristic of strong village social support networks incorporating family, relatives, neighbours and friends. Most of the newly-arrived Darfuri students strove to form new meaningful interpersonal relationships with ethnically similar Darfuris spending up to six hours a day sharing experiences of dislocation and memories of 'homeland' as a means to buffer against feelings of loss, isolation, distress, and despair. Also by frequently contacting the Darfuri student's union body, teachers, and counsellors who served as role models and gave assistance and accessibility to university services and facilities these Sudanese survivors of war gained a sense of comradeship and on-going encouragement and support from one another, to help them manage and cope with war-related distress, especially at times of bad news from the 'homeland' and current on-going urban-cultural challenges. Evidence suggests when war breaks down expansive social networks, interpersonal relationships and ethnically prescribed role models, other Sudanese affected by war will actively seek-out those with similar psychosocial stressors for support (Finch-Shakespeare \& Wickham, 2009; Marlowe, 2010).

Despite poor living conditions, difficulty in mastering academic obstacles, and even when some newly-arrived Darfuris felt humiliated, bothered and embarrassed because of perceived racism, subtle discrimination, stereotyping, and ridiculing, most participants did not portray a sense of victimhood nor were they frightened and trapped inside their hostels. In fact the data seemed to suggest a cultural context which informed their coping strategies. The Darfuri students turned to prayer and faith, becoming more resolute in their perseverance to succeed academically. Indicated by tribe and birth order which underlie; the role of socialization their interpretation of themselves as survivors and change agents for the improvement of their futures and the advancement of their 'homeland' may emphasize prescribed cultural norms, roles, and customs of inner strength and resilience to cope and adapt.

\section{Limitations}

It is the uniqueness and specificity of this present study's population that may in fact be its pitfall. Being afforded the opportunity to complete their high school education, attain 
a certificate, leave Darfur in times of war by plane or bus, able to get into the university system in Omdurman, and become university students, denotes a certain 'advantage'. This current sample may be non-representative of the wider population of Darfuri girls whose schooling may be interrupted as a result of being within the war zone or within an IDP camp. The study is further limited by its small sample size; and clearly generalizations to other female Sudanese affected by war, such as southern Sudanese, whether at AUW or within the general public are impossible to make, however given its exploratory nature, the study's findings may only serve to speculate on broader inferences; which this present research has attempted to do.

\section{Recommendations for future research and practices}

Ensuring a peaceful future for the Sudan would suggest catering for its female population affected by war. While the current study is, to the best of our knowledge, the first of its kind within the context of Darfuri female university students, it is envisioned that with a larger sample of Darfuri female measurements for the prevalence of psychological distress, in particular post-traumatic stress disorder, depression and anxiety may be quantified.

Of additional importance for future research may be to undertake quantitative research to investigate the evidence which suggests the equal role shared by war-related exposures and current on-going life challenges and stressors on emotional distress amongst Sudanese affected by war (De Jong et al., 2001). As such it is possible to understand Darfuris affected by war beyond a trauma-focused approach by investigating the differences and/or similarities between the mental health of war affected Sudanese (WAS) and non-WAS female university students in relation to PTSD, depression and anxiety, which may help consider the need for the development of a counselling unit with expert personnel in specialized trauma and psychosocial counselling skills (Miller \& Rasmussen, 2010) within the AUW in-house students counselling unit, presently unavailable within its services. 



\section{Chapter 3}

\section{Exposures to war-related traumatic events and post-traumatic stress disorder among displaced Darfuri female university students:}

An exploratory study

This chapter has been published as Badri, A., Crutzen, R., and Van den Borne, H.W. (2012) Exposures to war-related traumatic events and post-traumatic stress disorder among displaced Darfuri female university students: An exploratory study. BMC Public Health, 12, 603. 


\begin{abstract}
Background: With the deaths of hundreds of thousands and the displacement of up to three million Darfuris, the increasingly complex existing crisis in Darfur has warranted the need to investigate the current mental health of its civilian population. The purpose of this study is to assess post-traumatic stress disorder (PTSD) amongst a sample of displaced Darfuri female university students in Omdurman city and to investigate associated war-related trauma exposures. Methods: An exploratory cross-sectional study among Darfuri female university students $(\mathrm{N}=123)$ was conducted in February 2010. Dimensions of war-related traumatic exposures and prevalence and severity of PTSD sub-scales were measured using an adapted version of the Harvard Trauma Questionnaire (HTQ). Results: Correlation analysis was used to test the strength of association between dimensions of war-related traumatic exposures and PTSD outcome sub-scores. The findings indicate that $42 \%$ reported being displaced and participants have been exposed to an average of 28 war-related traumatic events either as victims or as witnesses ( $S D=14.24$, range 0 - 49 events). Also, there was a strong association between the experiential dimension of war-related trauma exposures and the full symptom of PTSD. Moreover, the refugee-specific self-perception of functioning sub-scale within the PTSD measurement scored a mean of $3.2(\mathrm{SD}=.56)$, well above the 2.0 cut-off. Conclusions: This study provides evidence of substantial traumatic war-related exposures that have resulted in a serious mental health burden among the Darfuri female student population in Omdurman. Urgent psychological assistance is required for this potentially marginalized group in the form of a comprehensive psychosocial trauma intervention.
\end{abstract}




\section{Introduction}

Increasingly complex, the current crisis in Darfur is sparked by a combination of factors; increased competition for dwindling environmental resources, extended periods of drought, disputes over grazing rights, land use, and augmented poverty. Since early 2003 ethnic and tribal tensions encouraged the militarization of small loosely organized groups rendering local clashes increasingly bloody. Indiscriminate attacks on villages not only killed and injured civilians but looted homes, demolished infrastructure, wells and irrigation systems, destroyed property such as farmland: livelihoods and potential for recovery destroyed. With an original population of 6 million inhabitants, approximately 2.74 million are displaced of which 1.88 million are sheltering in over 127 internally displaced camps throughout Darfur, and although numbers become vague, the estimate of those killed have reached the one million mark (De Waal, 2009; United Nations, 2005).

Those who have survived such war-related traumatic events are likely to bear the burden of horror, which might be reflected in their mental health. Post-traumatic stress disorder (PTSD) is the most recognized anxiety disorder that may result from direct exposures to war-related traumatic events. PTSD encompasses symptoms of intrusive recollections and flashbacks of the traumatic event, irritability, startled or exaggerated behaviours, emotional numbing, and feelings of detachment and avoidance behaviours (American Psychiatric Association, 1994). Trauma-focused research assumes that PTSD is primarily the direct result of exposures to armed conflict and being within conflict settings (Farhood et al., 1993; Fox \& Tang, 2000; Sarraj, Punamaki, Salmi, \& Summerfield, 1996).

In post-conflict and conflict-ridden societies, the prevalence rates of PTSD amongst civilians affected by war have been shown to be relatively high. In four post-conflict societies, namely, Ethiopia, Cambodia, Palestine, and Algeria, PTSD prevalence was found ranging from $15.8 \%$ for Ethiopia to $37.4 \%$ for Algeria (De Jong et al., 2001). Three studies have shown relatively high rates of PTSD amongst the civilian Southern Sudanese population with rates of $36 \%, 48 \%$, and $46 \%$ respectively; (Karunakara et al., 2004; Neuner et al., 2004; Roberts, Damunda, Lomoro, \& Sondrop, 2009). Likewise, PTSD has been documented in a number of recent studies within internally displaced peoples (IDP) camps in Darfur and has recorded relatively high rates amongst Darfuri civilians (Hamid \& Musa, 2010; Kim, Torbay, \& Lawry, 2007; Rasmussen \& Annan, 2009).

Given the momentum that the present Darfuri crisis is taking, very little is available on the mental health of Darfuri women and girls per se. This seemingly marginalized group of Sudanese civilians affected by war has been historically understudied. The aim of the present study was to address this empirical gap by focusing on the salient aspects of war-related experiences and to measure PTSD amongst a sample of undergraduate 
female Darfuri students currently studying at Ahfad University for Women (AUW) in Omdurman city, Sudan. In light of previous research conducted amongst war affected Sudanese, this group reflects the ongoing Darfuri war, and represents those having fled IDP camps and are now trying to cope and adjust as students in another part of the Sudan.

\section{Methods}

\section{Setting and participants}

AUW has an estimated 6839 students from all parts of the Sudan; these include students from conflict and post-conflict areas of the west and south. Subsequent to ethical approval by the AUW ethics committee in February 2010, Darfuri student lists were made available from the registrar's office, which contained contact information including year group, school allocation, class locations and course timetables, and showed a total of 209 registered students originating from the state of Darfur. In accordance with research objectives, a set of inclusion criteria were established: Darfuri born and raised; all members of her extended family must be of Darfuri origin; parents, siblings and extended family must still reside in Darfur; she had been in Darfur up to at least 2003 (war broke out in 2003; massive destruction and displacement followed); and she had no prior visits to Omdurman (her first visit to Omdurman was to continue her education). In total, 176 Darfuri students fit the inclusion criteria. Twenty of them already served in a previously conducted exploratory study (Badri, Crutzen, \& Van den Borne, 2011) and fifteen participated in the pre-testing of the measurement. This resulted in 141 Darfuri students being invited to participate in this study. A total of 123 (88\% response rate) Darfuri students participated in the current study with 18 (12\% nonresponse rate) potential participants unavailable at the allocated time because of conflict with their study schedules.

\section{Research team and data collection procedures}

The research team was made up of eleven AUW staff members and divided into two sub-teams; six field team members and five members on the instrument translation and adaptation team. Two senior members of staff served in a supervisory capacity overseeing the data collection procedure, providing debriefing sessions, and time-scheduling logistics, while the principal researcher and three staff members were responsible for the actual data collection and meetings with the student participants. The translation and adaptation team, included the in-house counsellor, a clinical psychologist from the school of psychology, two bilingual translators and the bilingual/bicultural principal researcher. Initial meetings with all members of the research team began on February 4 
2010 and lasted for three weeks. The aim of these meetings was to introduce the research objectives, target population, rationale and history of the questionnaire, give detailed discussions of each item in the questionnaire, and plan for instrument translation, adaptation, pre-testing and data collection within the allocated timeframe, which was scheduled to start in March of that year. Field-team meetings focused on establishing a contextual framework within which the data collectors were trained, which also included increasing an awareness of the ethno-graphic qualities and experiences of the target population, being attentive and vigilant for signs of distress among susceptible Darfuri students, and keeping in mind the sensitive nature of some questions. Each data collector was assigned a school and given a copy of the registrars list of student names. Introductory meetings with the students were made to ascertain availability and willingness to participate and to then agree upon exact dates, times and places for subsequent questionnaire administration. Thereafter s/he would meet the students, distribute the questionnaires and provide clarification if needed, and collect the finished questionnaires. Students were again informed of the voluntary nature of their participation, were assured confidentiality and were requested to sign a standard informed consent sheet on the covering page of the booklet. Depending on the actual volume of Darfuri students within a particular school, on average there was a show of 7 students per session, with each session taking approximately 45 minutes to an hour to complete. Data collection concluded on April 172010.

\section{Instruments}

The questionnaire booklet was comprised of two sections. An ethno-socio-demographic section in which data were collected on participants characteristics, such as place and date of birth, familial geographical origin, name of village and location to nearest town, tribe name, parental main source of livelihood, displacement characteristics (for example, property loss, parental loss of livelihoods, and date since displacement), date of arrival and current address in Omdurman city.

In the second section, the Harvard Trauma Questionnaire (HTQ) (Mollica et al., 1992) was used to measure the types of war-related traumatic events and severity of PTSD. It is an instrument that has been widely acknowledged to contain characteristics of cross-cultural adaptability that is important in reflecting context specific descriptions of war-related traumatic events of the community in which it is administered (Halepota \& Wasif, 2001; Kleijn, Hovens, \& Rodenburg, 2001; Shoeb, Weinstein, \& Mollica, 2007; Silove et al., 2007). Furthermore, it is a comprehensive screening assessment of PTSD where culturally relevant expressions of PTSD symptoms are accurately measured. To this end, and for the purposes of the present study, the self-report Parts I and IV of the HTQ were adapted and analyzed to become the Darfuri version of the HTQ. 
The adaptation of Part I of the HTQ began by undertaking in-depth interviews with twenty AUW Darfuri female undergraduate students in December 2009. A core checklist of 20 context specific war-related experiences was compiled from this reference group of students, accurately describing common acts of violence, combat situations and other war-related traumatic events. These events were: 'forced to hide', 'constant insecurity', 'movement in search of shelter', 'forced separation from family', 'distance from family', 'fleeing/running away', 'innocent victims being killed', 'finding dead bodies and buying them', 'living in displaced camps', 'burning of home', 'not having a home', 'owning nothing', 'complete change of life', 'aerial bombardment', 'hearing of atrocities', 'loss of health', 'increased physical ailments', 'suddenly gone missing', 'parentless children/teenagers', and 'seeing dead bodies' (Badri et al., submitted). A further twentynine items were selected from two existing versions of the HTQ: Indochinese and the Japanese (Mollica et al., 1992; Mollica et al., 1995). These items were added because they were cited by the reference group of Darfuri students and reflected important contextual details. A total of 49 war-related trauma items (20 Darfuri items added onto the existing 29 items) made up Part I of the Darfuri HTQ version.

In accordance with the guidelines set forth by the HTQ, participants were presented with this list of forty-nine potentially traumatic war-related events and were requested to endorse individual events according to four options $(\mathrm{E}=$ experienced, $\mathrm{W}=$ witnessed, $\mathrm{H}=$ heard about, or $\mathrm{N}=$ "no"). Fox and Tang (2000) noted that the 'experience' option reflected a person's direct exposure to war-related trauma. An additional level of intensity and severity would be to focus on two experiential levels of the HTQ, i.e., 'experience' and 'witness', of war-related traumatic events.

Part IV was the HTQ screening checklist for post-traumatic stress disorder (PTSD). The first 16 items measure PTSD according to the DSM-IV criteria (American Psychiatric Association, 1994); these remained the same. Only one of the two dissociative symptom items was omitted: 'feeling as if you are split into two and one of you is watching what the other is doing' because this was misunderstood repeatedly by the pre-test group and was not endorsed by the reference group. The other dissociative symptom item; 'finding out or being told that you have done something that you cannot remember', remained. This made a total of 17 PTSD items based on the DSM-IV criteria.

In addition, a twenty-nine-item refugee-specific symptom checklist to measure personal perceptions of psychosocial functioning was used. Based on the interviews previously conducted with the reference group, twenty-two culturally and contextually verifiable stressors due to violence and displacement were identified. These included: 'feeling miserable', 'feeling hopeless', 'only feeling normal among other Darfuris who also suffered the same sort of trauma', 'feeling no trust in others', 'feeling humiliated by your experiences' and 'spending time thinking about why these events happened to you'. Seven items were derived from the Japanese version of the refugee-specific symptoms 
checklist, which included: 'feeling as if you are going crazy', 'feeling isolated because of loss of relationships with family', 'feeling overly dependent on others', and 'feeling discriminated against because of loss of home'. Participants were asked to mark 1 for 'not at all', 2 'a little', 3 'quite a bit', and 4 'extremely' for the resultant number of 46 items.

Based on the guidelines set forth (Flaherty et al., 1988; Guillemin, Bombardier, \& Beaton, 1993), the translation and adaptation team undertook the task of translating the HTQ Darfuri version from English to classical Arabic (as opposed to colloquial Sudanese Arabic, as not all Darfuris speak the same dialect while all students are educated and can efficiently read and understand classical Arabic). Semantic equivalence was established using a bilingual probe technique to verify linguistic accuracy and meaning to the original English text. Back-translation of the Arabic version, and pre-testing among a group of fifteen AUW Darfuri students not in the original sample of this study but representing similar ethno-demographic characteristics showed high concordance with their understanding of the HTQ Darfuri version and stated that modifications were unnecessary.

AUW Ethic Committee provided ethical approval for the study in 2010. An information sheet was presented where objectives and procedures were clearly stated. Each participant completed a consent form at the onset of data collection to ensure informed consent. All data collected was confidential and anonymous, and securely stored by the research team.

\section{Analysis}

All data were analysed using the Statistical Package for the Social Sciences (version 17.0 for Windows). Means and standard deviations have been used to illustrate the severity of war-related exposure dimensions and sub-scales of PTSD. Pearson's correlations were conducted to test for the strength of association between dimensions of warrelated trauma exposures and sub-scales of PTSD symptoms. The significance level was set at $\mathrm{p}<0.01$ and two-tailed test values were reported. Strengths of association between variables, where Pearson's correlation coefficients are calculated to represent: $.10=$ weak; $.30=$ moderate; $.50=$ strong (Rosenthal, 1996).

To highlight the experiential level for each of the 49 items of war-related traumatic events the 'experienced' and 'witnessed' options were recoded into one value: ' 1 ' indicating the experience and/or witness of trauma; and ' 0 ' indicating heard about or no trauma experience. A sum score was created for all 49 items known as the experienced and $\backslash$ or witnessed cumulative dimension 'EW'. To assess the underlying factorial structure of war-related exposures the responses were then subjected to Principal Component analysis extraction. After Varimax factor rotation, a four factor model, known as war exposures dimensions, was chosen based on the Scree test and Eigen values $>1$. The 
first dimension, combat situations with violence, included 13-items ( $\alpha=.91)$, the second dimension included 7-items $(\alpha=.84)$ referring to the experience of material loss, the third dimension with 12 -items $(\alpha=.89)$ concerned the loss of relatives through killing, kidnapping or forced separation, and the fourth dimension with 8-items $(\alpha=.88)$ referred to forced displacement and related health threats.

Scoring of PTSD was according to the original instrument guidelines and standards (R. Mollica, McDonald, Massagli, \& Silove, 2004). Mean scores were calculated to establish 'checklist positive' for PTSD with a critical cut-off score at $>2.0$ which was previously determined as indicative of PTSD amongst war affected Sudanese (Roberts, Damunda, Lomoro, \& Sondrop, 2009). Internal consistency was calculated from the mean scores for the complete scale of PTSD ( $\alpha=$.93). As expected the DSM-IV criteria for PTSD showed a high internal consistency within each sub-scale: re-experiencing sub-scale $(\alpha=.70)$, avoidance/numbing sub-scale $(\alpha=.73)$, and psychological arousal sub-scales $(\alpha$ $=.81$ ), as did the refugee-specific self perception of psychosocial functioning sub-scale $(\alpha=.91)$.

\section{Results}

\section{Ethno-socio-demographic characteristics}

Ranging in ages between 15-25 years, participants came from Darfur to Omdurman city to continue their education at Ahfad University for Women. Currently $80 \%$ live in Omdurman city at one of the eight university hostels, while $20 \%$ live with relatives in either Khartoum or Bahri cities.

A substantial portion of participants $(45.5 \%)$ were from the Rizaigat, Habbaniya and BeniHalba Arab tribes of south-eastern Darfur. Their parents' main source of livelihood consisted of raising cattle and some subsistence farming in the villages on the outskirts of the two main towns of Nyala and AlDeain. AlZhaghawa, a non-Arab tribe of the northernmost town of ElFashir were represented by $23.6 \%$ of the participants whose parents mainly herded camels or had small businesses. The African AlFur tribes of the Bargu, Brnu, Tngr, Massaleet and Gumria represented 28\%. Inhabiting the western and central belt of Darfur, their parents' main subsistence was sedentary farming in the surrounding villages of AlGinaena, Kuttum, Murnei and Zalengaih.

\section{War-related traumatic exposures}

Coinciding with the 2003 onset of the current Darfuri conflict, the current sample have reported being personally experiencing and/or witnessing a mean of $28.2(S D=14.24)$ war-related traumatic events out of a possible 49. About two-thirds of the students have observed combat situations as they witnessed someone being killed, have seen dead 
bodies, and witnessed beatings to head and body. As a direct result of combat situations more than half of the students and their parents have lost goods, property and livestock. Nearly $60 \%$ had family members or friends who disappeared or were kidnapped. And $42 \%$ reported being forcibly removed or denied access to their homes compelling them to flee their villages and suffering related unhygienic conditions, and loss of health. Table 1 shows the factor loadings for each of the four dimensions found: combat, material loss; family loss; and displacement as well as the experiential level per war-related traumatic event. 
Table 1. Experiential level of war-related traumatic exposures

\begin{tabular}{|c|c|c|c|}
\hline War-related traumatic events & $\mathrm{n}$ & $\%$ & $\begin{array}{l}\text { Factor } \\
\text { loading }\end{array}$ \\
\hline \multicolumn{4}{|l|}{ Combat dimension (Range $=0-13$ ) } \\
\hline Movement in search of shelter & 55 & 44.7 & .588 \\
\hline Forced to hide & 85 & 69.1 & .633 \\
\hline Combat situation (e.g. shelling and grenade attacks) & 53 & 43.1 & .697 \\
\hline Arial bombings (planes and helicopters) & 67 & 54.5 & .684 \\
\hline Looting of nearby villages & 56 & 45.5 & .782 \\
\hline Forced evacuation under dangerous conditions & 67 & 54.5 & .785 \\
\hline Innocent victims being killed & 73 & 59.3 & .776 \\
\hline Hearing of atrocities & 67 & 54.5 & .716 \\
\hline Seeing dead bodies & 80 & 65.0 & .607 \\
\hline Finding dead bodies and burying them & 82 & 66.7 & .720 \\
\hline Forced to destroy someone else's property or possessions & 95 & 77.2 & .583 \\
\hline Witness beatings to head or body & 75 & 61.0 & .724 \\
\hline Prevented from burying someone & 94 & 76.4 & .716 \\
\hline \multicolumn{4}{|l|}{ Material loss dimension (Range $=0-7$ ) } \\
\hline Lack of shelter/ no where to live & 63 & 51.2 & .708 \\
\hline Confiscation or destruction of personal property & 72 & 58.5 & .764 \\
\hline Burning of home & 74 & 60.2 & .808 \\
\hline Not having right to a home & 72 & 58.5 & .846 \\
\hline Owning nothing & 63 & 51.2 & .806 \\
\hline Complete change of life & 47 & 38.2 & .558 \\
\hline Extortion \& robbery & 60 & 48.8 & .655 \\
\hline \multicolumn{4}{|l|}{ Family loss dimension (Range $=0-12$ ) } \\
\hline Imprisonment & 90 & 73.2 & .707 \\
\hline Kidnapped & 95 & 77.2 & .642 \\
\hline Suddenly gone missing & 81 & 65.9 & .640 \\
\hline Parentless children/ teenagers & 39 & 31.7 & .626 \\
\hline Disappearance or kidnapping of family member or friend & 73 & 59.3 & .797 \\
\hline Forced separation from family members & 78 & 63.4 & .710 \\
\hline Distance from family & 54 & 43.9 & .518 \\
\hline Enforced isolation from others & 73 & 59.3 & .679 \\
\hline Knifing/ axing/ slaughtering & 58 & 47.2 & .729 \\
\hline Harshness and cruel treatment ( physical or mental suffering) & 86 & 69.9 & .639 \\
\hline $\begin{array}{l}\text { Serious physical injury of family member or friend due to combat situation } \\
\text { or landmine }\end{array}$ & 69 & 56.1 & .785 \\
\hline Murder, or death due to violence, of family member or friend & 71 & 57.7 & .788 \\
\hline
\end{tabular}




\begin{tabular}{lcccc}
\hline War-related traumatic events & $\mathrm{n}$ & $\%$ & $\begin{array}{c}\text { Factor } \\
\text { loading }\end{array}$ \\
\cline { 1 - 2 } Combat dimension (Range=0-13) & & & & \\
\cline { 1 - 3 } Displacement dimension (Range=0-8) & 47 & 38 & .723 \\
\cline { 1 - 2 } Lack of food or water & 55 & 44.7 & .782 \\
Living in displaced camps & 57 & 46.3 & .738 \\
Exposed to unhygienic conditions & 52 & 42.3 & .817 \\
Poor health without access to medical care & 69.5 & 44.7 & .779 \\
Loss of health & 70.3 & 44.7 & .697 \\
Increase in physical ailments & 64.4 & 47.2 & .765 \\
Exposed to threats, humiliation, or discrimination & 63.6 & 65.9 & .616 \\
\hline
\end{tabular}

The severity of war-related traumatic events experienced by the current sample is illustrated in Table 2. A mean of $7.7(\mathrm{SD}=4.0)$ combat situations were experienced; a mean of $3.2(\mathrm{SD}=2.2)$ material loss events; $7.1(\mathrm{SD}=3.9)$ family loss events were experienced; and a mean of $3.8(\mathrm{SD}=2.8)$ displacement events.

Table 2. Descriptive statistics regarding severity of war-related trauma exposures

\begin{tabular}{lccc}
\hline Dimension & Range & Mean & SD \\
\hline Cumulative experienced \& witnessed & $0-49$ & 28.28 & 14.24 \\
Displacement & $0-8$ & 3.87 & 2.78 \\
Combat & $0-13$ & 7.77 & 3.98 \\
Family loss & $0-12$ & 7.16 & 3.86 \\
Material loss & $0-7$ & 3.20 & 2.16 \\
\hline
\end{tabular}

Table 3 shows the correlations between the four dimensions of war-related traumatic exposures and the cumulative experiential dimension. The cumulative dimension of war-related traumatic exposures was highly correlated with its sub-dimensions: combat situations ( $\mathrm{r}=.93)$; material loss ( $\mathrm{r}=.81)$; family loss $(\mathrm{r}=.92)$; displacement dimension $(\mathrm{r}=.86)$.

Table 3. Correlations of war-related trauma exposures dimensions

\begin{tabular}{lcccc}
\hline & Displacement & Combat & Family loss & Material loss \\
\hline Cumulative experienced \& witnessed & $.860^{* *}$ & $.933^{* *}$ & $.923^{* *}$ & $.814^{* *}$ \\
Displacement & & $.750^{* *}$ & $.771^{* *}$ & $.694^{* *}$ \\
Combat & & & $.802^{* *}$ & $.704^{* *}$ \\
Family loss & & & & $.687^{* *}$ \\
\hline
\end{tabular}

${ }^{* *}$ Correlation is significant at the 0.01 level (2-tailed). 


\section{Prevalence for post-traumatic stress disorder \& refugee-specific perceptions of psychosocial functioning}

The results indicate that $80.9 \%$ of the present Darfuri student sample met DSM IV criteria for post-traumatic stress disorder with a cut-off score of $>2.0$ (Roberts, Damunda, Lomoro, \& Sondrop, 2009). Furthermore, the refugee-specific self perception of functioning sub-scale tends to show the highest percentage above cut-off $(89.7 \%)$ and the highest score (M=3.2, $\mathrm{SD}=.56)$ as shown in Table 4.

Table 4. Descriptive Statistics PTSD sub-scales

\begin{tabular}{lccccc}
\hline & DSM-IV PTSD & $\begin{array}{c}\text { Re- } \\
\text { experiencing }\end{array}$ & Avoidance/numbing & $\begin{array}{c}\text { Psychological } \\
\text { arousal }\end{array}$ & $\begin{array}{c}\text { Refugee-specific } \\
\text { self perception of } \\
\text { functioning }\end{array}$ \\
\hline Mean & 2.99 & 2.82 & 3.17 & 2.89 & 3.23 \\
Median & 3.00 & 2.75 & 3.28 & 3.00 & 3.36 \\
SD & .63 & .80 & .62 & .80 & .56 \\
Minimum & 1.20 & 1.00 & 1.17 & 1.00 & 1.24 \\
Maximum & 4.00 & 4.00 & 4.00 & 4.00 & 4.00 \\
\% above cut-off & 80.9 & 70 & 88.7 & 73 & 89.7 \\
\hline
\end{tabular}

Overall PTSD measurement was highly correlated with its sub-scales (re-experiencing $(\mathrm{r}=.815)$; avoidance and numbing $(\mathrm{r}=.896)$; psychological arousal $(\mathrm{r}=.899)$ and with refugee specific self-perception of functioning $(\mathrm{r}=.860)$ (Table 5).

Table 5. Correlations between PTSD sub-scales

\begin{tabular}{|c|c|c|c|c|}
\hline & DSM-IV PTSD & $\begin{array}{l}\text { Re- } \\
\text { experiencing }\end{array}$ & $\begin{array}{l}\text { Avoidance/ } \\
\text { numbing }\end{array}$ & $\begin{array}{l}\text { Psychological } \\
\text { arousal }\end{array}$ \\
\hline \multicolumn{5}{|l|}{ DSM-IV PTSD } \\
\hline Re-experiencing & $.815^{* *}$ & & & \\
\hline Avoidance/numbing & $.896^{* *}$ & $.574^{* *}$ & & \\
\hline Psychological arousal & $.899^{* *}$ & $.640^{* *}$ & $.712^{* *}$ & \\
\hline $\begin{array}{l}\text { Refugee-specific self perception of func- } \\
\text { tioning }\end{array}$ & $.860^{* *}$ & $.668^{* *}$ & $.780^{* *}$ & $.792^{* *}$ \\
\hline
\end{tabular}

${ }^{* *}$ Correlation is significant at the 0.01 level (2-tailed).

The strength of association between PTSD sub-scales with exposure dimensions shows a general trend of a strong association between all PTSD sub-scales with all war-related traumatic events. Family loss seems to stand out as the strongest association with PTSD and its sub-scores, while material loss seems to be moderately associated (Table 6). 
Table 6. Association between PTSD sub-scale scores and Exposure dimensions

\begin{tabular}{lccccc}
\hline Dimension & DSM- & \multicolumn{2}{c}{ Re- } & \multicolumn{2}{c}{ Avoidance/ Psychological Refugee-specific } \\
& IV & PTSD & arousal & $\begin{array}{c}\text { self perception of } \\
\text { functioning }\end{array}$ \\
\hline Cumulative experienced \& witnessed & $.542^{* *}$ & $.531^{* *}$ & $.502^{* *}$ & $.406^{* *}$ & $.526^{* *}$ \\
Displacement & $.514^{* *}$ & $.514^{* *}$ & $.459^{* *}$ & $.391^{* *}$ & $.488^{* *}$ \\
Combat & $.422^{* *}$ & $.425^{* *}$ & $.410^{* *}$ & $.286^{* *}$ & $.425^{* *}$ \\
Family loss & $.614^{* *}$ & $.573^{* *}$ & $.563^{* *}$ & $.488^{* *}$ & $.599^{* *}$ \\
Material loss & $.407^{* *}$ & $.373^{* *}$ & $.397^{* *}$ & $.303^{* *}$ & $.441^{* *}$
\end{tabular}

${ }^{* *}$ Correlation is significant at the 0.01 level (2-tailed).

\section{Discussion}

This study is - to our knowledge - the first attempt to describe and quantify war-related traumatic exposures and to assess the severity of PTSD symptomology amongst a sample of displaced Darfuri women trying to build up a new life and resettle as students in another part of the Sudan. The results indicate an additional echelon of intensity associated with the multiple types of exposures being endorsed across the board by this current sample of Darfuri participants. The high prevalence rate of war-related traumatic events is consistent with prior research concerning Sudanese experiences of warrelated traumatic events (Hamid \& Musa, 2010; Karunakara et al., 2004; Rasmussen et al., 2010; Roberts, Damunda, Lomoro, \& Sondrop, 2009).

The experiences of having lived through war-related traumatic events and the fact that a little over $80 \%$ of the current sample of female Darfuri student participants scored in the clinical range of PTSD is an unfortunate reality. The data presented in this paper supports the argument that symptoms of traumatisation continue to be a burden on mental health, even after a time lapse. While there should be an acknowledgement of the distress experienced by persons living with memories of war-related events, it appears that the elevated distress is not uncommon and may be faced by other Sudanese IDP groups (Hamid \& Musa, 2010; Roberts, Damunda, Lomoro, \& Sondrop, 2010; Rasmussen \& Annan, 2009), however, the members of the current sample show a much higher rate of PTSD, stressing an urgent need for psychological assistance.

Affected by their exposures and experiences and their current levels of PTSD, the data obtained from this sample suggests that items relating to the avoidance sub-scale need not necessarily be a maladaptive response. Avoidance behaviors, thoughts, feelings or activities are in affect a reduction of the disturbing memory and is a therapeutic objective in many forms of psychological interventions aimed at reducing the effects of traumatic events (Kagee, 2005; Sarraj, Punamaki, Salmi, \& Summerfield, 1996). Fur- 
thermore, previous qualitative data indicates that this population may continue to experience symptoms of PTSD, even though their salience may be obscured by more immediate and current concerns of daily life stressors, such as financial worries, urbancultural adaptation and academic challenges (Badri, Crutzen, \& Van den Borne, 2012a).

There is evidence to suggest that psychosocial interventions aimed at ameliorating PTSD amongst survivors of war-related trauma is well placed and can be applied in preventive, rehabilitative and reconstructive spheres. Psycho-education, skills training, and relief activities such as games, arts and sports are some ways shown to have significance with Bosnian youth (Betancourt, 2005). Participatory approaches in the form of mentored community development activities, skills building and teaching appropriate coping strategies have been suggested to facilitate the reintegration of Eritrean youth (Farwell, 2001). Research among Sudanese refugees in Canada seem to suggest a process of cultural adjustment whereby psychological and social resources are met based on traditional coping strategies and customary social support networks (Simich, Este, \& Hamilton, 2010). Furthermore, Meffert and Marmar (2009) found a culturally informed version of Interpersonal Therapy (IPT) to be effective in the treatment of PTSD and depression among Darfuri refugees in Cairo (Meffert \& Marmar, 2009).

Further research is needed to answer the question of which combination of multilevel approaches can be successfully integrated as a model of psycho-social intervention that best provides support and counseling amongst this population of Darfuri female students. A culturally appropriate multi-level approach can be designed and implemented by AUW. Trained trauma counselors providing a buffer against the stigmatization of PTSD and mental health may also serve to supervise peer and social support groups counseling sessions that aim at ameliorating the memory of war-related traumatic events.

\section{Limitations}

There are a number of limitations to this study. Firstly, using a single institute may have resulted in a fairly homogenous group, i.e., university students as opposed to the general Darfuri displaced population, although our sample comprised of fairly diverse ethnic groups, and our aim was to target female university students, characteristically an understudied and marginalized group in Sudanese society. Secondly, although our study included measures for PTSD, it would have been worthwhile in adding depression and anxiety as measurements for a complete picture of mental health description. Nevertheless, for the purpose of this explorative study, PTSD was the key outcome measure of interest. Finally, we must stress that our data only provide evidence regarding an association between war-related traumatic events and PTSD, not a causal relation. Further 
analysis should be undertaken before any firm conclusions may be established, although a causal relation would be a matter of course.

\section{Conclusions}

We believe, therefore, that our results offer an addition to identifying the experiential level of war-related traumatic exposures and recognizing the potential risks of mental health outcomes, in particular PTSD, amongst a Darfuri population of university aged Sudanese women that require urgent psychosocial intervention. 



\section{Chapter 4}

\section{Anxiety, depression and resilience among war-affected and non-war- affected Sudanese female university students: A comparative study}




\begin{abstract}
Objective: To investigate and compare mental health status between war-affected Darfuri and non-war-affected Omdurmani undergraduate students at Ahfad University for Women in Omdurman city, Sudan. Method: A cross-sectional study was used to assess and compare the anxiety, depression (HSCL-25) and resilience scores (Resilience Scale) and related demographic variables for 116 Darfuri and 299 Omdurmani students. Results: Overall anxiety, depression and resilience scores varied minimally between Darfuri and Omdurmani students. Depression scores were negatively related with resilience scores, but not with anxiety scores. Younger participants (15-20 years) and those who had a parent deceased were more anxious, while those whose father was a bluecollar worker (indicating low socio-economic status) were more depressed, but also more resilient. Furthermore, participants whose tuition fees were paid by their parents (indicating a higher socio-economic status) scored lower on resilience.
\end{abstract}




\section{Introduction}

With the growing Sudanese Diaspora, whether as internally displaced people (IDPs) or as refugees, the increasing body of literature has highlighted the issue of pre and post displacement concerns on the poor mental health status among survivors of war. Posttraumatic stress disorder (PTSD), generalized anxiety disorder and depression are the most frequent pre-displacement manifestations of mental health problems among Sudanese (Peltzer, 1999), while, post-displacement concerns of current on-going life stressor suggest an impact on elevated levels of psychosocial maladjustment within a host community (Schweitzer, Melville, Steel, \& Lacherez, 2006). Recent studies assessing Darfuri IDP women's mental health concluded that 31\% - 38\% exhibited signs of major depression symptoms (Kim, Torbay, \& Lawry, 2007; Morgos, Worden, \& Gupta, 2008). Furthermore, Rasmussen and colleagues (2010) and Hamid and Musa (2010) have acknowledged that both pre-displacement factors and post-displacement stressors such as daily social and material pressures contributed to the mental health burden among Darfuri IDPs.

Furthermore, anticipatory anxiety symptoms amongst those not directly exposed nor displaced by war occur in much the same way as war survivors (Thabet, Abed, \& Vostanis, 2002). The possibility of associated factors such as the occurrence of daily life stressors (Paardekooper, De Jong, \& Hermanns, 1999), economic, social and cultural disruptions and changes in their ecological systems (Bronfenbrenner, 1979) have also predicted a higher than expected prevalence of anxiety and depression symptoms amongst urban populations, particularly amongst women (Broadhead \& Abas, 1997; Lundberg, Cantor-Graae, Rukundo, Ashaba, \& Östergren, 2009).

Additional stressors of everyday life related to social problems, finance and budgeting, accommodation adjustments, and academic challenges have been shown to increase the risk of anxiety disorders and depressive episodes among university students (Bramness, Fixdal, \& Vaglum, 1991; Dahlin, Joneborg, \& Runeson, 2005; Hysenbegasi, Hass, \& Rowland, 2005; Tomoda, Mori, Kimura, Takahashi, \& Kitamura, 2000; Vaz, Mbajiorgu, \& Acuda, 1998). Furthermore, anxiety and depression have been associated with differences in ethnic backgrounds. Some studies comparing ethnically diverse undergraduates report no differences (Constantine, Chen, \& Ceesay, 1997; Lester \& DeSimone, 1995) while others report that there are between ethnicities in relation to depression and anxiety (Aldwin \& Greenberger, 1987; Bertocci, Hirch, Sommer, \& Williams, 1992). The mixed findings necessitate further investigation, which has been done in the current study by comparing two groups that differ in ethnic background.

Resilience has been linked to the personal qualities and predispositions of Sudanese war traumatized populations. Their continued capacity both immediately and in the months following exposure has consistently shown a process for successful adapta- 
tion as the most common outcome trajectory despite varying stressors and symptom levels (Badri, Crutzen, \& Van den Borne, 2012a; Goodman, 2004; Khawaja, White, Schweitzer, \& Greenslade, 2008; Schweitzer, Greenslade, \& Kagee, 2007; Tipping, Bretherton, \& Kaplan, 2007).

The association between mental health problems and positive resilience outcomes amongst undergraduates demonstrate their consistent need for achievement, perseverance, and high resilience levels; which speak to the ability of undergraduates in utilizing these personal characteristics in their attempt to effectively cope with current stressors (Hall, Spruill, \& Webster, 2002; Work, Parker, \& Cowen, 1990).

The findings in a preceding qualitative study amongst Darfuri war-traumatized AUW students gave an expanded view of daily confrontations with academic challenges, life hassles, and urban-cultural clashes and also explored their habitual coping strategies used to deal with these stressors (Badri, Crutzen, \& Van den Borne, 2012a). Published literature on generalized anxiety disorder, depression symptoms and resilience scores among female university students in the Sudan is nonexistent. The aim of the present study is to investigate and compare measures of anxiety, depression and resilience among war-affected Darfuri and non-war-affected Omdurmani undergraduate female students at Ahfad University for Women in Omdurman city, Sudan. These groups do not only differ in terms of being affected by war, but also in terms of ethnic background.

\section{Methods}

\section{Participant Selection}

AUW has an estimated 6839 female-only students from all parts of the Sudan; these include students from conflict and post-conflict areas of the west and south. Subsequent to ethical approval by the AUW ethics committee in February 2010, students' lists were made available from the registrar's office, which included year group, school allocation, class locations and course timetables, and showed a total of 209 registered students originating from the state of Darfur and 511 Omdurmani registered students. In accordance with research objectives, a set of inclusion criteria were established: born and raised in their respective place of origin; all members of her extended family must be of Darfuri/Omdurmani origin; and parents, siblings and extended family must still reside in the place of origin. As for the Darfuri students they must have been in Darfur up to at least 2003 (war broke out in 2003; massive destruction and displacement followed); and had no prior visits to Omdurman (their first visit to Omdurman was to continue her education). As for the Omdurmani students, they had not left Omdurman city for any length of time. In total, 176 Darfuri and 310 Omdurmani students fit the inclusion crite- 
ria and were invited to participate in this study. Thirty-four Darfuri students were unavailable at the allocated time because of conflict with their study schedules, and twenty-six questionnaires were discredited for incomplete data: actual participants from Darfuri were 116 students. Eleven of the Omdurmani questionnaires were discredited because of incompletion resulting in a total of 299 Omdurmani students.

\section{Instruments}

The questionnaire comprised three sections. An ethno-socio-economic demographic section in which data were collected on participants characteristics, such as date of birth, ethnicity and tribe name, name of catchment (Omdurmani students) and village (Darfuri students), composition of the family, parental death, parental occupation, parental property ownership and source for payment of tuition fees. A set of proxy indicators were included to determine socio-economic factors: age; father's death; father's occupation as blue or white collar job (as opposed to mother's occupation since approximately $90 \%$ of mothers were housewives and clearly fathers were the main breadwinners for the family); owning property at place of origin (whether in building, land, farm land or livestock); and source of payment of tuition fees (parents, self, scholarship, or other family member).

Section two of the booklet included the Hopkins Checklist-25 (HSCL-25; (Hesbacher, Rickels, Morris, Newman, \& Rosenfeld, 1980). Consistent with the DSM-IV criteria, this version consisted of ten items from the anxiety cluster, fifteen items from the depression cluster and two additional somatic symptoms (poor appetite; difficulty asleep or staying asleep). The HSCL-25 has demonstrated its usefulness and has been employed in several studies including the screening of depression and anxiety in normal populations (Derogatis, Lipman, Rickels, Uhlenhuth, \& Covi, 1974), and in refugee and displaced populations (Mollica, Wyshak, de Marneffe, Khoun, \& Lavelle, 1987), and in comparisons between and across cultures and contexts (Cepeda-Benito \& Gleaves, 2000). A recent research undertaken among a Sudanese sample (Roberts, Ocaka, Browne, Oyok, \& Sondrop, 2009) validated the original HSCL-25 and identified a cut-off score of 1.75 to detect clinically significant symptoms of generalized anxiety disorder and depression symptoms. This version has been translated into Arabic, known as 'Juba Arabic' (Roberts, Ocaka, Browne, Oyok, \& Sondrop, 2009); however, a decision was made to initiate the translation process anew within the present samples' Sudanese context. The translation of the HSCL-25 was undertaken by the translation team according to the standard guidelines of instrument translation (Jones, Lee, \& Phillips, 2001): translated and back-translated; resolution of discrepancies through discussions; and compared with the original English version in order to establish semantic equivalence (a pre-requisite condition for reliability). 
The final section of the questionnaire included the 25 -items Resilience Scale (Wagnild \& Young, 1993) to specifically measure the multidimensional aspects of resilience. The scale includes five different aspects of resilience: perseverance, equanimity, meaningfulness, self-reliance and existential aloneness. Scores range from 25-175; those greater than 145 indicate moderately high to high resilience, 125-145 indicate moderately low to moderate levels of resilience, and scores of 120 and below indicate low resilience. The Resilience Scale has been used with a wide range of populations of different ages, socioeconomic, and educational backgrounds.

\section{Research team and data collection procedures}

The research team was made up of eleven AUW staff members and divided into two sub-teams; six field team members and five members on the instrument translation team. Two senior members of staff served in a supervisory capacity overseeing the data collection procedure, providing debriefing sessions, and time-scheduling logistics, while the principal researcher and three staff members were responsible for the actual data collection and meetings with the student participants. The translation team included the in-house counsellor, a clinical psychologist from the school of psychology, two bilingual translators and the bilingual/bicultural principal researcher. Initial meetings with all members of the research team began on February 42010 and lasted for three weeks. The aim of these meetings was to introduce the research objectives, target populations, rationale and history of the HSCL-25 and the RS, give detailed discussions of each item in the questionnaire booklet, and plan for instrument translation, and data collection within the allocated timeframe, which was scheduled to start in March of that year.

Each data collector was assigned a school and given a copy of the registrars list of student names. Introductory meetings with the students were made to ascertain availability and willingness to participate and to then agree upon exact dates, times and places for subsequent questionnaire administration. Thereafter $\mathrm{s} / \mathrm{he}$ would meet the students, distribute the questionnaires and provide clarification if needed, and collect the finished questionnaires. Students were again informed of the voluntary nature of their participation, were assured confidentiality and were requested to sign a standard informed consent sheet on the covering page of the booklet. Depending on the actual volume of students within a particular school, on average there was a show of 7 students per session, with each session taking approximately 45 minutes to an hour to complete. Data collection concluded on April 17 2010. To assure overall data quality, standard protocols were followed to ensure consistency in the entering and coding of data. Routine comparisons were made between the hard copy data forms and the keyed data to reduce input errors. Periodic edits were performed on the computer database to safeguard against out-of-range entries. 


\section{Analysis}

Descriptive analyses were performed to investigate the distribution of our data. Principle component analyses (PCA) were conducted for the anxiety, depression and resilience items separately. Items with factors loadings $<.40$ on all components were left out. Internal consistencies were assessed by means of Cronbach's alpha. Pearson correlation coefficients were used to assess correlations between anxiety, depression and resilience scores. Subsequently, $\chi^{2}$-tests and independent samples t-tests were used to assess differences between Darfuri and Omdurmani participants in terms of using place of origin, age, one of the parents deceased, paying for tuition fees, occupation of the father, and whether their parents owned property at their place of origin as well as depression, anxiety, and resilience scores. Furthermore, linear regression analyses were conducted using place of origin, age, one of the parents deceased, source of tuition fees, fathers' occupation, and whether their parents owned property at their place of origin as predictors. Separate analyses were run, using depression, anxiety and resilience scores as dependent variables. Backward procedures were used to create final parsimonious models. All analyses were conducted using SPSS 17.

\section{Results}

A total of 415 participants completed the measurements; 116 (28\%) of them were Darfuri and 299 (72\%) were Omdurmani. Sixty-one percent ranged between 15-20 years of age and thirty-seven percent between 21-25 years. Twenty-one percent had one of their parents deceased. Tuition fees were paid by parents $(74 \%)$, relatives $(17 \%)$ or by means of a scholarship (9\%). Seventy-two percent of the fathers were blue-collar workers, ten percent were white-collar workers and the remaining eighteen percent were retired or unemployed. In their place of origin, $57 \%$ of parents owned property.

PCA regarding anxiety items revealed that there was one factor $(\alpha=.86)$ underlying these items (Eigenvalue $=4.48$ ), which explained $44.8 \%$ of the total variance. PCA regarding depression items revealed that there was one factor $(\alpha=.86)$ underlying these items (Eigenvalue $=5.26$ ), which explained $35.1 \%$ of variance. None of the factor loadings regarding anxiety or depression were $<.40$. PCA regarding resilience items revealed that four items had a factor loading $<.40$. Removing these items resulted in one factor $(\alpha=.90)$ underlying these items (Eigenvalue $=7.16$ ), which explained $32.5 \%$ of variance.

Fifty-six percent and 51\% of the Darfuri sample manifested symptoms of anxiety and depression respectively, while Omdurmani students showed $54 \%$ and $58 \%$ of anxiety and depression respectively. The most commonly endorsed anxiety symptoms amongst both Darfuri and Omdurmani groups were headaches (84\%), feeling fearful 
(80\%), feeling restless and tense (73\%), while depression symptoms mostly reported were self-blame (79\%), feeling blue (78\%), feeling no interest in usual activities (72\%), and everything is an effort (89\%).

Fifty-seven percent of the total sample indicated a moderate to moderately low level of resilience, with very little variance between Darfuri and Omdurmani mean scores (5.3 and 5.1, respectively).

Anxiety scores were positively correlated with depression scores $(r=.68, p<.001)$, but they were not correlated with resilience scores $(r=-.09, p=.08)$. Depression scores, however, were negatively correlated with resilience scores $(r=-.19, p<.001)$.

Table 1 provides an overview of the differences between Darfuri and Omdurmani participant characteristics. The Darfuri participants were older, were more likely to have one of their parents deceased, were less likely to have their parents pay for tuition fees, were more likely to have a father working in a blue-collar job, and their parents were less likely to have owned property in their place of origin. There were no differences between Darfuri and Omdurmani participants in terms of anxiety, depression, and resilience scores.

Table 1. Personal and psychological characteristics: Comparisons differences between Darfuri and Omdurmani participants.

\begin{tabular}{|c|c|c|c|c|}
\hline Measure & $\begin{array}{c}\text { Total } \\
(\mathrm{N}=415)\end{array}$ & $\begin{array}{c}\text { Darfuri } \\
(\mathrm{n}=116)\end{array}$ & $\begin{array}{l}\text { Omdurmani } \\
(\mathrm{n}=299)\end{array}$ & Test \\
\hline Age & & & & $\chi^{2}=6.57, p=.04$ \\
\hline $15-20$ years [\%] & 61 & 51 & 64 & \\
\hline $21-25$ years [\%] & 37 & 43 & 33 & \\
\hline $26+$ years $[\%]$ & 2 & 6 & 3 & \\
\hline One of parents deceased [\%] & 21 & 28 & 18 & $\chi^{2}=4.99, p=.03$ \\
\hline Tuition fees paid by [\%] & & & & $\chi^{2}=30.58, p<.001$ \\
\hline Parents & 74 & 59 & 80 & \\
\hline Relatives & 17 & 20 & 16 & \\
\hline Scholarship & 9 & 22 & 5 & \\
\hline Occupation father [\%] & & & & $\chi^{2}=9.25, p=.01$ \\
\hline Blue-collar & 72 & 82 & 68 & \\
\hline White-collar & 10 & 2 & 13 & \\
\hline Retired/unemployed & 18 & 15 & 19 & \\
\hline Owned property [\%] & 57 & 48 & 61 & $\chi^{2}=4.93, p=.03$ \\
\hline Anxiety [M (SD)] & $1.90(0.57)$ & $1.95(0.71)$ & $1.88(0.51)$ & $\mathrm{t}=0.92, p=.36$ \\
\hline Depression [M (SD)] & $1.94(0.55)$ & $1.87(0.61)$ & $1.96(0.53)$ & $\mathrm{t}=-1.40, p=.16$ \\
\hline Resilience [M (SD)] & $5.35(1.07)$ & $5.50(1.07)$ & $5.30(1.06)$ & $\mathrm{t}=1.68, p=.09$ \\
\hline
\end{tabular}


Table 2 provides an overview of the final regression models. In line with the independent-samples t-tests, geographical background/place of origin (as an indicator for urbanrural living) was not significant in any of the final models. With regard to anxiety, participants aged between 15-20 years and those who had one of their parents deceased scored lower on anxiety. Participants whose father was a blue-collar worker (as a proxy indicator for family socio-economic status) scored lower on depression, but higher on resilience. With regard to resilience, participants whose tuition fees were paid by their parents scored lower on resilience. It needs to be stressed, however, that the variance explained by the final models was very limited: the included variables hardly had any predictive value regarding anxiety, depression or resilience scores.

Table 2. Standardized betas in final regression models for Anxiety, Depression and Resilience.

\begin{tabular}{llcc}
\hline Measure & Anxiety & Depression & Resilience \\
\hline Place of origin & & & \\
Age & $-.16^{*}$ & & \\
$15-20$ years & & & \\
$21-25$ years & $-.12^{*}$ & & $-.14^{*}$ \\
One of parents deceased & & & \\
Tuition fees paid by & & & $.12^{*}$ \\
$\quad$ Parents & & & \\
$\quad$ Scholarship & & $-.12^{*}$ & .03 \\
Occupation father & & & .01 \\
$\quad$ Blue-collar & & & \\
White-collar & -.10 & & \\
Owned property & .04 & & \\
\hline $\mathrm{R}^{2}$ & & & \\
\hline
\end{tabular}

${ }^{*} p$-values smaller than .05 .

\section{Discussion}

Interpreting the findings of this study need to be considered within a variety of contexts: pre and post displacement concerns amongst Darfuri war-affected students; and regarding the sample as a whole as undergraduate students in a least developed country. Published literature on generalized anxiety disorder, depression symptoms and resilience scores among female university students in the Sudan is nonexistent. Therefore comparing the data with representative national norms is virtually impossible. However, drawing on the findings of two preceding studies amongst this student sample and 
comparing the results from certain international studies relating to undergraduate students in terms of anxiety, depression and resilience may help interpretation of findings.

More than fifty percent of the participating war-affected Darfuri female undergraduates manifested symptoms of generalized anxiety disorder and major depression, with those Darfuris who had one of their parents deceased exhibiting more anxiety. Similar results for clinical symptoms of depression have been recorded amongst women in IDP camps in Darfur (Kim, Torbay, \& Lawry, 2007; Morgos, Worden, \& Gupta, 2008)), among other samples Darfuri IDPs (Hamid \& Musa, 2010; Souza, Yasuda, \& Cristofani, 2009), among Southern Sudanese (Coker, 2004b; Simich, Hamilton, \& Baya, 2006), and similarly can be found amongst war torn regions in Africa (Kagee, 2005; Tang \& Fox, 2001).

Furthermore, these war-related pre-displacement manifestations of mental health problems were further investigated in a preceding study where $80.9 \%$ of the current Darfuri sample met DSM-IV criteria for post-traumatic stress disorder (PTSD) symptoms, which were strongly associated with their experiential level of war-related traumas (Badri, Crutzen, \& Van den Borne, 2012b). Two-thirds of the current sample reported being survivors of and witnesses to a high number of war-related traumatic exposures including, combat situations, loss of parents and family members, loss of material possessions and displacement in IDP camps. The loss of their family intensified feelings of isolation, discrimination, and humiliation, survival guilt and shame (Badri, Crutzen, \& Van den Borne, 2012b). Moreover, post-displacement stressors were chronicled in the findings of a preceding qualitative study amongst Darfuri war-traumatized AUW students (Badri, Crutzen, \& Van den Borne, 2012a). Their transportation struggles as they find the means to travel to Omdurman in the pursuance of their education, their confrontation with hassles related to relocating to Omdurman city and their life at AUW, their academic challenges, urban-cultural clashes, and financial worries have all predicted a mental health burden on these young Sudanese women (Badri, Crutzen, \& Van den Borne, 2012a).

Nevertheless, the results show that there are no differences between Darfuri and Omdurmani participants in terms of anxiety and depression scores highlighting the possibility of anticipatory anxiety symptoms amongst the Omdurmani undergraduates (Thabet, Abed, \& Vostanis, 2002). The data also shows that more Omdurmanis are depressed than Darfuris, underscoring the prospect of associated factors such as the occurrence of daily life stressors (Rasmussen et al., 2010; Paardekooper, De Jong, \& Hermanns, 1999) in suggesting a higher than expected prevalence of anxiety and depression symptoms particularly amongst these urban undergraduates (Lundberg, CantorGraae, Rukundo, Ashaba, \& Östergren, 2009; Mumford, Minhas, Akhtar, \& et. al., 2000). The predictive variables of age, geographical background/place of origin (as an indicator for urban-rural living), a parent's death, source of payments of tuition fees, father's 
occupation (as an indicator for SES), and parents owning property in their place of origin played a limited role in determining comparisons between the ethnic diversities of Darfuris and Omdurmanis in relation to anxiety and depression scores (Constantine, Chen, \& Ceesay, 1997; Lester \& DeSimone, 1995). Moreover, younger participants and those who had one of their parents deceased were more anxious and those who were of a low socio-economic status were more depressed, indicating the possibility that the differences between Darfuris and Omdurmanis are less dramatic but more related to current stressful conditions of everyday life. The findings demonstrate that the pervasiveness of a low SES, including the potential impediment on parents to cover educational fees, costs, unemployed or blue collar workers, places a substantial burden on both groups of students to excel in order to justify the major financial sacrifices their families are making. Additional academic challenges, finance and budgeting problems, social stressors, accommodation adjustments and harsh living conditions (Badri, Crutzen, \& Van den Borne, in press) increases the risk for developing of anxiety disorders and depressive episodes (Tomoda, Mori, Kimura, Takahashi, \& Kitamura, 2000; Vaz, Mbajiorgu, \& Acuda, 1998).

Nonetheless, anxious and depressed Darfuri and Omdurmani students have demonstrated moderate to moderately low levels of resilience. Resilience has been linked to the personal qualities and predispositions of war traumatized Sudanese (Goodman, 2004; Tipping, Bretherton, \& Kaplan, 2007). The moderate to moderately low levels of resilience demonstrated by the Darfuri students reflects a pragmatic capacity to bounce back despite their adversity. Their cultural values and upbringing develops the potency for survival and fortitude given their harsh environmental surroundings (Rasmussen \& Annan, 2009; Rasmussen et al., 2010; Young et al., 2005), while purporting not to show its devastation. Also, being geographically distant from a war-torn Darfur and resettled in a more secure and stable environment (Omdurman city) may have served to increase their endurance and enhance their resilient expression, which relates to the significance of effective coping, endurance and resilience amongst war-affected Sudanese (Meffert \& Marmar, 2009; Simich, Hamilton, \& Baya, 2006; Stoll \& Johnson, 2007; Tipping, Bretherton, \& Kaplan, 2007). Furthermore, Darfuri students use religious practices and beliefs (praying and reading the Quran), form interpersonal relationships with other Darfuris, use social support networks, and a positive future outlook which seemed to lend to their ability to cope with war-related emotional distress, current on-going life hassles and urban-cultural challenges (Badri, Crutzen, \& Van den Borne, 2012a). Darfuri students have also endorsed items relating to the avoidance subscale of PTSD symptomology (Badri, Crutzen, \& Van den Borne, 2012b). Several researchers have argued that avoidance behaviours, thoughts, or feelings need not necessarily be a maladaptive responses; in fact it is considered to be effective in reducing the disturbing memory which is a therapeutic objective in many forms of psychological interventions (Kagee, 2005; Sarraj, 
Punamaki, Salmi, \& Summerfield, 1996). Their ability to avoid or numb their emotions and deal with their more immediate and current concerns of daily life stressors (Rasmussen et al., 2010), such as financial worries, urban-cultural adaptation and academic challenges by relying on the various coping strategies (Badri, Crutzen, \& Van den Borne, 2012a) is reflected in their resilient score and seems not to have interfered with life as an undergraduate student.

Fifty-seven percent of anxious and depressed Darfuri and Omdurmani students have demonstrated moderate to moderately low levels of resilience despite their ongoing stressors and low SES (Driscoll, 2006; Garmezy, 1991; Rew, Taylor-Seehafer, \& Thomas, 2001; Wyman, Cowen, Work, \& Parker, 1991). Female students in many less developed countries confer that education improves resilient factors and coping capability (Dodani \& Zuberi, 2000), while familial and extra-familial support systems (Christle, Harley, Nelson, \& Jones, 2007; D'Imperio, Dubow, \& Ippolito, 2000) have been shown to enhance resilience amongst low SES students. These students may use their resilient characteristics (Wagnild, 2009) to raise their self-efficacy, self-esteem, giving them a greater sense of control over stressful situations (Harpham, 1994).

This explorative and descriptive study included the limitations inherent to the use of a cross-sectional design; such as the difficultly to separate cause from effect between the variables in our study. The fact that we studied just one sector of the general population (university students) and one gender in one private university may limit the generalisability of the results. However, the exclusivity of investigating a female sector of the Sudanese society, addresses an empirical gap in relation to knowing about their mental health and resilience levels. The findings are hoped to serve as base line information concerning the mental status and resilience of Sudanese female undergraduate students. Furthermore, the lack of published research amongst this Sudanese community may have a two-fold disadvantage. On the one hand, the conceptualization of psychological symptoms and resilience amongst undergraduates has not been authenticated and on the other the instruments used to measure depression, anxiety, and resilience have not been culturally validated within this Sudanese context. This requires further research. However, this study, has followed instrument guidelines and protocol, and is envisaged to provide for absence of important empirical information on generalized anxiety and depression symptoms and resilience levels among female undergraduate students.

Contrary to much of the literature that established war affected individuals as being more depressed and anxious when compared to those not affected by war, the present study found that both war Darfuri and Omdurmani students displayed a similar burden of mental health problems. Both Darfuri and Omdurmani students endorsed equal numbers of anxiety and depression scores. Furthermore, the study is an important addition to the resilience literature in that it calls attention to the possibility that under con- 
ditions of cumulative stressor exposure and chronic stress, students in this sample portray a veneer of resilience.

With no national data available to support the findings, much remains to be done. Although, it is encouraging to find that although symptom levels of anxiety and depression are prevalent amongst both groups of students; resilience has consistently emerged as the most common outcome trajectory. However, it also calls attention to the possibility that under extreme stressor exposure, certain resilience protective characteristics may have a diminished impact on the prevalence of mental health status. Research which focuses on improving resilience characteristics and patterns of protective resources used to ameliorate stress among female undergraduate students may help shape the development of an appropriate intervention program which fosters the use of successful protective resources and enhances the utilization of effective resilient characteristics. 



\section{Chapter 5}

\section{Coping resources and resilience}

characteristics among war-affected and non-war-affected Sudanese female university students: A comparative study

"In order to succeed, people need a sense of self-efficacy, struggle together with resilience to meet the inevitable obstacles and inequalities of life."

Albert Bandura

This chapter has been submitted for publication as Coping resources and resilience characteristics among war-affected and non-war-affected Sudanese female university students: A comparative study. Badri, A. Crutzen, R. and Van den Borne, H. W. 


\begin{abstract}
This study explored and compared external coping resources and internal resilience characteristics among two ethno-geographically different Sudanese female undergraduates. War-traumatized, internally displaced, rural Darfuri students were compared with Omdurmani non-war traumatised, urban host students to investigate differences in effective coping to daily stressors in term of academic, social and financial matters. Implementing both internationally-used and culturally-grounded assessment tools a cross-sectional study of 116 Darfuri and 299 Omdurmani students were presented with an eight item checklist of external coping resources and five internal resilience characteristics to manage academic, financial and social/relationship stressors. Darfuris were more likely to use people of the same tribe and ethnicity as coping resource, while Omdurmanis were more likely to use friends and leisure activities as coping resources. Regarding internal resilience characteristics, the Darfuris relied more on meaning to cope with stressors in general. Darfuris were more anxious in comparison with Omdurmanis when resilience characteristics were taken into account. The Omdurmanis relied more on themselves for academic and financial/economical stressors and on equanimity and existential aloneness for social stressors. Each group of students used different external coping resources and possessed different resilience characteristics with regards to managing academic, financial and social/relationship stressors. In-house mental health services can benefit from this study when designing counselor training or intervention programs with regards to encouraging undergraduates to use their effective resilience characteristics and coping resources in their attempts to adapt and manage stressors.
\end{abstract}




\section{Introduction}

Given the unique setting of on-going war in its western province of Darfur the Sudan provides a distinctive context in which to appraise mental health disorders, stress sources, coping resource utilization and resilience characteristics. In earlier studies among Darfuri internally displaced persons (IDPs) the prevalence of major depression ranged between $31 \%-70 \%$ and post-traumatic stress syndrome (PTSD) ranged between 45\% - 75\% (Rasmussen \& Annan, 2009; Hamid \& Musa, 2010). More recently, Darfuri female university students exposed to traumatic war events and post-war social stressors were found to exhibit high levels of PTSD, anxiety, and depression; 81\%, 56\%, $51 \%$ respectively (Badri, Crutzen, \& Van den Borne, 2012b; Badri, Crutzen, \& Van den Borne, submitted).

With the persistent insecurity and unrest in Darfur a considerable spill over of IDPs has reached as far east as Omdurman city, some 1,200 kilometres away from the conflict area. Omdurman is home to Ahfad University for Women (AUW), an all female private university, with students from all parts of the Sudan, including Darfuris. A recent qualitative study identified war-related traumatic exposures, current on-going life stressors and coping strategies for Darfuri students (Badri, Crutzen, \& Van den Borne, 2012a). Daily life stressors and urban-cultural challenges increased the distress levels while prayer, religious beliefs, the use of available social support networks and making meaning of life asserted their ability to cope. Furthermore, a previous comparative study among Darfuri war affected and Omdurmani non-war affected AUW students indicated small variations and no significant differences in estimations of anxiety and depression prevalence between these two groups (Badri, Crutzen, \& Van den Borne, submitted). Resilience scores were also quite similar; verging between moderate - moderately low levels (Badri, Crutzen, \& Van den Borne, submitted). While anxiety, depression and resilience levels were comparable between war-affected Darfuris and non war-affected Omdurmanis, the process of meeting the challenges imposed by academic hurdles, the need to form new relationships and friendships, and for many the adjustment imposed by living away from home and family posits a myriad set of stressors on their undergraduate current lives (Vaz, Mbajiorgu, \& Acuda, 1998). Research has shown that the onset of depressive episodes (Dahlin, Joneborg, \& Runeson, 2005) may be triggered by academic performance pressures, the lack of learning materials (Kelly, Ryan, Altman, \& Stelzner, 1993; Rice, Herman, \& Petersen, 1993; Seidman et al., 1995), self-expectation and expectation from their significant 'others' (Moffat, McConnachie, Ross, \& Morrison, 2004; Sherina, Rampal, \& Kaneson, 2004b) and test anxiety (Hamad, 1974; Sharma, Parnian, \& Spielberger, 1983) among students from low to medium socio-economic status (SES) urban backgrounds, much like the current sample of Omdurmani female student. 
Challenges associated with rural students, such as those coming from the villages of Darfur, have demonstrated a plethora of psychological distress (Gillespie \& Noble, 1992). Navigating large ethnically and culturally diverse university campuses (Maltzan, 2006), the lack of exposure to technology such as photocopy machines, overhead projectors, computers (Pearson \& Sutton, 1999), and steering through busy city streets (Parsons, 1992), overwhelms a rural undergraduate causing an exacerbation of stress reactions (Swift, 1988).

African studies have associated student daily life stressors with low to medium SES demographic variables and have suggested that family financial strains (inability to pay tuition fees), and quality of life (overcrowded accommodation, inadequate infrastructure (water and electricity supply), transportation problems) were significantly related to the stress levels of Zimbabwean university students (Joseph, Chipandambira, Hungwe, \& Mupawose, 2007). Hamad (1974) found that family death, parental divorce or illness within the family, level of father's education, and past history of family mental illness were associated with levels of distress among Sudanese university students from low to medium SES.

Effective coping is achieved when stressful situations are accurately appraised and specific behavioral and psychological efforts are employed to manage, reduce, or tolerate stressful events (Saipanish, 2003; Sreeramareddy, Shankar, \& Binu, 2007).

The mobilization of strong protective external resources, such as social support networks (e.g., family and extrafamilial networks) can buffer against stressors (Waltz, 1994) and subsequent maladaptive responses (Benson \& Deeter, 1992; Cohen, 1991; DuBois, Felner, Brand, Adan, \& Evans, 1992; Licitra-Kleckler \& Waas, 1993) including depression (Dalgard, Dowrick, \& Lehtinen, 2006). Early studies have reported that rural residence utilized significantly more social support networks, than the urban residence when faced with both minor life stressors and daily hassles (Arotz-Badenan \& Olvin, 1986). Furthermore, students possessing a rich repertoire of coping resources such as emotional, social, physical, and financial are likely to cope more successfully with stressors (Gan, Zhang, Wang, Wang, \& Shen, 2006), while practising hobbies, participating in social and recreational activities, having future aspirations, and having more free time to practise these activities reduced stress substantially among students (Masten, Tušak, Zalar, \& Ziherl, 2009).

The ability to deal with life's stressors, cope effectively, and promote positive mental health outcomes suggests a process whereby internal resilience characteristics such as perseverance, self-reliance, meaning, equanimity, and existential aloneness (Wagnild \& Young, 1993) intertwines in multiple pathways with external social support networks (Masten, Best, \& Garmezy, 1990).

Research conducted to investigate the variables that promote resilience, enhance perseverance, and improve positive self-concepts among students from low SES families 
and racial/ethnic minorities, underscore the importance of, peer support as buffers against depression and anxiety (Luthar, 1991), having at least one caring relationship from extended family members (Werner, 1995), and the ability to actively seek social support from parents, peers and teachers (Driscoll, 2006). Furthermore, factors that promote academic resilience among urban poor students suggested that the personal attributes of persistency (inclination to stay on target to complete a task or chosen goal), responsibility, and attentiveness were related to academically successful low SES undergraduates (Valencia, 1994). A study on the role of parents in the development of educational resilience of their daughters showed that despite parents' low levels of educational and occupational attainment, they were able to foster a sense of resilience in their children and play an important role in their educational success and aspirations (Ceja, 2004).

Notwithstanding, the evidence that undergraduates can bounce back and achieve academic accomplishment in spite of vulnerability to emotional distress and possible psychological disorders such as anxiety and depression (Badri, Crutzen, \& Van den Borne, submitted), published research of Sudanese university students stressors, coping resources and resilience characteristics, is rare. Moreover, the study of women as an entity of their own with their atypical characteristics: traditionally marginalized; wartraumatized; displaced; resettled; and coming from low-income communities, are virtually undocumented. In light of this paucity in research, the present study sought to assess the coping resources and resilience characteristics for two ethno-geographical diverse female ethnicities. Academic, social/interpersonal, and financial strain were investigated in relation to coping resources habitually used and resilience characteristics, as well as to determine the relationship between and as well as determining the relationship with anxiety and depression scores.

\section{Methods}

\section{Participant Selection}

AUW has an estimated 6839 students from all parts of the Sudan; these include students from conflict and post-conflict areas of the west and south. Subsequent to ethical approval by the AUW ethics committee in February 2010, students' lists were made available from the registrar's office, which contained contact information including year group, school allocation, class locations and course timetables, and showed a total of 209 registered students originating from the state of Darfur and 511 Omdurmani registered students. In accordance with research objectives, a set of inclusion criteria were established: born and raised in their respective place of origin; all members of her extended family must be of Darfuri/Omdurmani origin; and parents, siblings and extended 
family must still reside in the place of geographical origin. As for the Darfuri students she must have been in Darfur up to at least 2003 (war broke out in 2003; massive destruction and displacement followed); and she had no prior visits to Omdurman (her first visit to Omdurman was to continue her education). As for the Omdurmani students, she has not left Omdurman city for any length of time. In total, 176 Darfuri and 310 Omdurmani students fit the inclusion criteria and were invited to participate in this study. Thirty-four Darfuri students were unavailable at the allocated time because of conflict with their study schedules, and twenty-six questionnaires were discredited for incomplete data: actual participants from Darfuri were 116 students. Eleven of the Omdurmani questionnaires were discredited because of incompletion resulting in a total of 299 Omdurmani students.

\section{Instruments}

The questionnaire was comprised of three sections. An ethno-socio-economic demographic section in which data were collected on participants characteristics, such as place and date of birth, familial geographical origin, ethnicity and tribe name, name of catchment (Omdurmani students) and village (Darfuri students), composition of the family, parental death, parental occupation, parental property ownership and source for payment of university tuition fees. Place of geographical origin was set as a proxy indicator to determine urban-rural upbringing and socioeconomic status was based on parental occupation (blue/white collar jobs; and, according to Sudanese societal norms were characterized 'high-middle' representing occupations requiring higher education, relatively more economically rewarding and associated with higher prestige, e.g. engineers, teachers, nurses, businessmen, civil servants; and 'middle/low' representing farmers, unskilled workers, self-employed, or unemployed.

\section{Coping resources}

An eight item checklist of coping resources was developed to determine student's habitual use of resources when faced with acute stressors defined as major life events and transition such as war exposures or chronic stressors such as daily hassles and minor lifestyle changes. The resources were: family; tribe/ethnicity; friends; students union; role models; and the AUW counseling service; religion (spirituality); and leisure activities (recreation and hobbies). These resources were based on a previous qualitative exploratory study on coping with current daily life stressors (Badri, Crutzen, \& Van den Borne, 2012a). These habitual coping resources were then coded into eight categories, to construct a coping resource scale. Participants were asked to mark from 0 (not used) to 3 (mostly used) for each coping resource. The sum scores were then computed ranging from 0-24. 


\section{Resilience characteristics}

Based on the theoretical definition of resilience, five resilience characteristics were derived from the Resilience Scale developed by Wagnild and Young (1993): self reliance; meaning; equanimity; perseverance; and existential aloneness. Self-reliance is identified as the most important characteristic which lays the foundation for the other four (Wagnild, 2009). Meaning connotes making meaning of life experiences and a purpose in life; perseverance, self-reliance, equanimity and existential aloneness confer with the concept of inner strength promoting effective coping and a 'coming home to yourself' (Wagnild, 2009). In three open-ended questions, participants had to describe how they cope with academic (e.g., level of difficulty and volume of course requirements, availability of learning materials, examination anxiety); financial (e.g., lack of material resources/possessions/utilities, poor living conditions/hostels, tuition fees, living expenses, and budgeting allowances); and social/relationship stressors (university campus community/ethnic-cultural diversity, alienated/estranged, family quarrels, and friendship misunderstandings). Their answers were then coded by two researchers into the five resilience characteristics classification according to Wagnild (1993). Discrepancies regarding coding were resolved by means of discussion.

\section{Procedure}

The registrar's office submitted participants name, departments and timetables of both Darfuri and Omdurmani participants. These participants were then contacted by the research field team and initial discussion for questionnaire administration time was organised. A brief explanation of the study and its components was given at the beginning of the administration session. Participants were advised that names were not to be recorded and that the information received was strictly confidential. Once written consent was obtained participants were invited to complete the questionnaire. Depending on the actual volume of students within a particular school, on average there was a show of 7 students per session, with each session taking approximately 45 minutes to an hour to complete. Data collection concluded on April 172010.

\section{Analyses}

Differences in coping resources items between Darfuri and Omdurmani participants were tested by means of independent samples t-tests. Subsequently, principal component analyses (PCA) were conducted for coping resource items and used to construct a coping resource scale. Independent samples t-tests and $\chi^{2}$-tests were used to assess differences between Darfuri and Omdurmani participants in terms of the coping resource scale and resilience characteristics respectively. These differences were taken into account as predictors in linear regression models upon place of origin. Separate 
analyses were run using anxiety and depression scores as dependent variables. Backward procedures were used to create final parsimonious models. All analyses were conducted using SPSS 17.

\section{Results}

A total of 415 participants completed the measurements; 116 (28\%) of them were Darfuri and 299 (72\%) were Omdurmani. Sixty-one percent ranged between 15-20 years of age and 37\% percent between 21-25 years. Twenty-one percent had one of their parents deceased. Tuition fees were paid by parents (74\%), relatives $(17 \%)$ or by means of a scholarship (9\%). Seventy-two percent of the fathers were blue-collar workers, ten percent were white-collar workers and the remaining eighteen percent were retired or unemployed. In their place of origin, $57 \%$ of parents owned property.

Table 1 shows the differences in coping resource items between Darfuri and Omdurmani participants. Darfuri were more likely to use people of the same tribe and ethnicity as coping resource, while Omdurmani were more likely to use friends and leisure activities as coping resources. There were no differences with regard to the other coping resources (e.g., family, counseling service). PCA regarding coping resource items revealed one factor $(\alpha=.83$ ) underlying these items which explained $46.4 \%$ of the total variance. All items had factors loadings > .55 .

Table 1. Means, standard deviations, and t-tests for differences in coping resource items for students from Darfur and Omdurman.

\begin{tabular}{lccc}
\hline Coping resource & $\begin{array}{c}\text { Darfuri } \\
(\mathrm{n}=116)\end{array}$ & $\begin{array}{c}\text { Omdurmani } \\
(\mathrm{n}=299)\end{array}$ & Test \\
& $\mathrm{M}(\mathrm{SD})$ & $\mathrm{M}(\mathrm{SD})$ & \\
\hline Family & $2.41(1.08)$ & $2.47(0.86)$ & $\mathrm{t}=0.55, p=.59$ \\
Tribe/ethnicity & $1.34(1.06)$ & $1.01(0.65)$ & $\mathrm{t}=-3.13, p=.002$ \\
Friends & $1.97(1.13)$ & $2.31(0.84)$ & $\mathrm{t}=2.91, p=.004$ \\
Student union & $0.99(0.90)$ & $0.98(0.67)$ & $\mathrm{t}=-0.09, p=.93$ \\
Role models & $1.86(1.32)$ & $1.94(1.16)$ & $\mathrm{t}=0.56, p=.58$ \\
Religion & $1.22(1.07)$ & $1.17(0.87)$ & $\mathrm{t}=-0.34, p=.73$ \\
Counseling service & $1.41(1.13)$ & $1.26(0.94)$ & $\mathrm{t}=-1.29, p=.20$ \\
Leisure activities & $1.60(1.10)$ & $1.93(1.01)$ & $\mathrm{t}=2.83, p=.005$
\end{tabular}

Table 2 shows that there were no differences between students from Darfur and from Omdurman on the coping resource scale. Darfuri and Omdurmani participants did differ, however, in terms of the resilience characteristics they possessed for academic, fi- 
nancial and social/relationship stressors. For all of these, the Darfuri relied more on meaning. The Omdurmani relied more on themselves for academic and financial stressors and on equanimity and existential aloneness for social/relationship stressors.

Table 2. Independent samples t-tests and $\chi^{2}$-tests differences between Darfuris and Omdurmanis stressors in relation to coping resources and resilience characteristics.

\begin{tabular}{lcccc}
\hline Measure & $\begin{array}{c}\text { Total } \\
(\mathrm{N}=415)\end{array}$ & $\begin{array}{c}\text { Darfuri } \\
(\mathrm{n}=116)\end{array}$ & $\begin{array}{c}\text { Omdurmani } \\
(\mathrm{n}=299)\end{array}$ & Test \\
\hline Coping resource scale [M (SD)] & $13.0(5.2)$ & $12.8(6.6)$ & $13.1(4.5)$ & $\mathrm{t}=0.42, p=.68$ \\
Academic stressors & & & & $\chi^{2}=17.92, p<.001$ \\
$\quad$ Self reliance [\%] & 33.9 & 20.3 & 37.7 & \\
Meaning [\%] & 12.0 & 23.2 & 8.9 & \\
Equanimity [\%] & 3.2 & 2.8 & 4.3 & \\
Perseverance [\%] & 50.6 & 50.6 & 50.7 & \\
Existential aloneness [\%] & 0.3 & 0.0 & 1.4 & \\
Financial stressors & & & & \\
Self reliance [\%] & 37.4 & 23.1 & 40.6 & \\
Meaning [\%] & 16.7 & 30.8 & 13.5 & \\
Equanimity [\%] & 33.5 & 34.6 & 33.2 & \\
Perseverance [\%] & 12.1 & 11.5 & 12.2 & \\
Existential aloneness [\%] & 0.4 & 0.0 & 0.4 & \\
Social/relationship stressors & & & & \\
Self reliance [\%] & 23.4 & 26.1 & 22.8 & \\
Meaning [\%] & 34.7 & 58.7 & 29.7 & \\
Equanimity [\%] & 21.5 & 4.3 & 25.1 & \\
Perseverance [\%] & 6.0 & 6.5 & 5.9 & \\
Existential aloneness [\%] & 14.3 & 4.3 & 16.4 & \\
\hline
\end{tabular}

Table 3 shows the predictors in linear regression analysis between Darfuri and Omdurmanis in relation to the two dependent variables of anxiety and depression scores. Darfuri students were more anxious in comparison with Omdurmanis when resilience characteristics were taken into account. The use of meaning as a resilience characteristic for financial stressors reduced anxiety scores, which was used more often by Darfuri (Table 2). Participants that were self reliant regarding social/relationship stressors had decreased depression scores. 
Table 3. Standardized betas in final regression models for anxiety and depression scores .

\begin{tabular}{lcc}
\hline Measure & Anxiety & Depression \\
\hline Place of origin & $.32^{*}$ & \\
Academic stressors & & \\
Self reliance & & \\
Meaning & & \\
Equanimity & & \\
Perseverance & & \\
Financial stressors & $-.21^{*}$ & \\
Self reliance & & $-.18^{*}$ \\
Meaning & & \\
Equanimity & & \\
Perseverance & & \\
Social/relationship stressors & & \\
Self reliance & & \\
Meaning & & \\
Equanimity & & \\
Perseverance & & \\
\hline $\mathrm{R}^{2}$ & & \\
\hline$*$-values smaller than 05.11 &
\end{tabular}

\section{Discussion}

In line with the study aims there are differences between how Darfuris and Omdurmanis utilize external coping resources and possess internal resilience characteristics to manage, cope and adapt to academic, financial and social/relationship stressors. While acknowledging that the participants of the current study are of Sudanese origin, and that clearly coping resources and resilience characteristics had an ameliorating effect on measures of anxiety and depression there are, nevertheless, striking differences between the 'extra-ordinary' major life stressors experienced by the Darfuri participants (war-affected) and those 'normal' tertiary stressors (non-war-affected) experienced by the Omdurmanis.

In the shadows of their war-related experiences and post-displacement stressors (Badri, Crutzen, \& Van den Borne, 2012b), Darfuris' resilience levels (Badri, Crutzen, \& Van den Borne, submitted) demonstrate an ability of altering extreme emotional negativity to thinking positively by a process of continuous reappraisal and reinterpretation (Wortman, Battle, \& Lemkau, 1997). The ability to engage in meaning as an internal resilient characteristic when faced with social/relationship, academic and financial stressors reflects a capability to address future difficulties more assertively (Knight, 
2006). Furthermore, the high endorsement of this resilience characteristic within the Darfuri group signifies an interaction that draws from their repertoire of spirituality, religious practice, sense of appreciation, gratitude and thankfulness of being alive (Badri, Crutzen, \& Van den Borne, 2012a) in an attempt to make meaning not only of the psychological effect of war exposure but also the context of the experience in their current lives indicating an urgency to adjust to university life, and an importance to successfully accomplish current goals; notwithstanding the pursuant of educational opportunity.

Incongruent cultural/ethnic mannerisms, differences in language patterns, the challenges of navigating academic hurdles, and financial worries exemplify social/relationship, academic and financial stressors among the Darfuri-rural ethnic minority (Badri, Crutzen, \& Van den Borne, 2012a), which may have had the potential of increasing anxiety and depressive symptoms (Badri, Crutzen, \& Van den Borne, submitted). The coping resource of seeking contact with other Darfuris from the same ethnicity or tribal background emphasizes the importance of group cohesion and positive ethnic identity (Holleran \& Waller, 2003; Werner, 1995) that buffers against stressors much like other rural student communities (McIntire, Marion, \& Quaglia, 1990) and is paramount within the Darfuri culture (Badri, Crutzen, \& Van den Borne, 2012a). Moreover, financial stressors are somewhat alleviated by the fact that all Darfuri students are provided with a tuition scholarship from AUW (Badri, Crutzen, \& Van den Borne, 2012a) and seems to act as a protective buffer against psychological distress (Timmins \& Kaliszer, 2002). Finally, social support networks and their interpersonal relationships with 'significant others' (Cope, 1972; Duru \& Poyrazli, 2007; Swift, 1988) continuously affirm their resilience characteristic of meaning and tends to determine how Darfuris cope with their new urban and student life at AUW.

Resilience characteristics endorsed by the Omdurmani non-war-affected students that help them cope with social/relationship, academic, and financial stressors were mainly their ability to rely on themselves, equanimity, and existential aloneness. The group has also reported that largely due to the effects of a chronic disadvantaged environment, being categorized within a low socio-economic status, faced with family financial pressures, and a pervasive lack of resources (Badri, Crutzen, \& Van den Borne, submitted), they still have the capacity for self-reliance by taking on jobs optimizing their income, and spending wisely only on pertinent possessions, boosts competence and self-worth (Werner \& Smith, 1992).

Although the most salient familial and extrafamilial protective coping resources may be a strong force in promoting resilience and encouragement (Ceja, 2004), they may themselves be compromised by a chronically disadvantaged environment, availability of economic opportunities and resources (Dubow, Edwards, \& Ippolito, 1997), and the debilitating effects of poverty may serve an additive function to metal distress 
levels (Seidman et al., 1995; Seidman, Allen, Aber, Mitchell, \& Feinman, 1994), including depression (Assimeng, 1981; Badri, Crutzen, \& Van den Borne, submitted; Nagase et al., 2009), and may lose their protective impact (Wyman, Cowen, Work, \& Parker, 1991).

Academic stressors are eased by communicating and seeking information, guidance, and support from significant others, such as university staff, including counsellors. Their familiarity with environmental surroundings, people, and cultural mannerisms, function as alleviators of daily life stressors by interacting with fellow Omdurmani students and pursuing leisure activities as a coping resource. Their active participation in extra-curricular activities including, AUW's team sports, and choir and music bands as recreational activities suggests the multiple pathways to resilience (Masten, Best, \& Garmezy, 1990). Committing to these activities recognizes their resilience characteristic of equanimity, 'taking things in their stride', 'finding something to laugh about' and 'taking things one day at a time', and fosters a practice of commitment in future aspirations (Masten, Best, \& Garmezy, 1990).

On the other hand, Omdurmanis also indicated that they would rather be alone (i.e., 'existential aloneness') as a resilience characteristic and had lower needs for social relationships (Iwasaki, 2003) than Darfuri students. It is important to highlight the significance of this resilience characteristic as a 'coming home to yourself' (Wagnild, 2009), a preferred coping strategy to moderate Omdurmanis academic, social/relationship and financial stressors (Abela \& Hankin, 2009).

Several limitations of the current study should be noted. First, as the current study was cross-sectional in nature, conclusions about the directions of the reported associations cannot be drawn. Longitudinal research designs are needed to determine whether coping resources and resilience characteristics influence the subsequent development of anxiety and depressive symptoms, and stressors or whether the onset of symptoms and stressors negatively impacts the types of coping strategies students employ. The differences between Darfuri and Omdurmani participants in terms of coping resources and resilience strategies, however, are independent of the cross-sectional design. Secondly, although studying coping in university students is a topic of great significance, the current findings cannot be generalized to, for example, male undergraduate students. However, the premise for this particular research was to target female university students, characteristically an understudied and marginalized group in Sudanese society. Further research is thus needed to examine whether the current findings replicate in other university samples. Thirdly, there are countless ways of measuring stressor variables for example, acculturative stress and peer pressure. However, based on previous studies among these samples, academic, social/relationship and financial stressors were the most pertinent. Additional research may discover other relevant stressor variables. 
This study provides valuable insights reflecting the trends by which two distinctly diverse groups of students utilize coping resources and exhibit resilience characteristics in the face of academic, financial and social/relationship stressors. According to the results, Darfuris were more anxious in comparison with Omdurmani students when resilience characteristics were taken into account. Their minority representation as a ethnicculture within a larger Omdurmani dominated ethnic-culture at AUW prompted them to identity with those who are ethno-culturally similar and hence endorse a high level of social support and solidarity. Also, their individual capacity for meaning as an inner resilience characteristic endorses their flexibility in the face of academic, financial and social/relationship stressors and challenges. Omdurmani students, more pragmatic, 'whatever it takes', tendency of self reliance protects them against academic and financial stressors, as they feel they can 'handle', 'manage', and 'get through difficult times' on their own. Also, Omdurmanis were more likely to use friends and leisure activities as coping resources, and relied on a more urban-type equanimity and existential aloneness for social stressors. Recognizing that undergraduate students have a reservoir of potential positive responses despite experiencing adversity, including war-related exposures and low SES, suggests the importance of establishing a framework within which to develop an effective counseling service that identifies existing resilience characteristics and effective coping resources as those found within this sample of Darfuri and Omdurmani students. The implications for trauma counselors may be a need to re-train in essential differential paradigms, including, re-conceptualizing traumatic reactions as reactions to adversity, rather than on the limiting conceptualization of PTSD, generalized anxiety disorder and major depression symptoms; attributing meaning, equanimity and existential aloneness, as resilience characteristics; disempowering helplessness in the therapeutic relationship; and focusing on the eudemonic (meaning and purpose) of well-being (Papadopoulos, 2002). 



\section{Chapter 6}

\section{Promoting Darfuri women's psychosocial health: Developing a war- trauma counsellor training program}

"The mind is driven by one's own mission and responsibility to safeguard against domination and to that which enriches prosperity."

Yousif Badri

This chapter has been accepted for publication as Badri, A. Crutzen, R., Eltayeb, S. and Van den Borne, H. W. (2013). Promoting Darfuri women's psychosocial health: Developing a war-trauma counsellor training program. EPMA Journal 


\begin{abstract}
Women are considered special groups uniquely vulnerable in the context of war exposures. To effectively target the resources aimed at mitigating their mental health consequences, and optimizing and maximizing the use of mental health provisions, culturally relevant war-trauma counsellor training is required. The objective of this study is to provide evidence- and theory- based guidelines for developing a war-trauma counsellor training program in the Sudan, mainly based on qualitative and quantitative studies among war-affected Darfuri female students. Cultural conceptualizations such as gender roles and religious expectations as well as theories that emphasize resilience and other psychosocial adaptation skills have been operationalized to reflect the totality of the Darfuri women's experiences. Furthermore, the results of four interrelated studies among war-traumatized Darfuri internally displaced undergraduate women informs the basis that guides an outline for qualification development, capacity building and skills consolidation among Sudanese mental health care providers. Explicit war-related psychosocial needs assessment tools, specific war-related trauma counsellor training and particular counsellor characteristics, qualities, and awareness are recommended that pertain to strengthening the efficacy of war trauma Sudanese counsellors. The aim is to produce expertly trained war-trauma counsellors working with war-affected Darfuri women in particular and with regards to their helpfulness in responding to the psychosocial needs of war-exposed Sudanese in general.
\end{abstract}




\section{Introduction}

Epidemiological studies have suggested that women are more likely than men to experience mental health consequences as a direct result of war trauma (Basoglu, Salcioglu, \& Livanou, 2002; Kessler, Sonnega, Bromet, Hughes, \& Nelson, 1995) with a twofold greater lifetime prevalence for PTSD than men (Breslau, Davis, \& Andreeski, 1997; Perkonigg, Kessler, \& Storz, 2000), and demonstrate higher rates of psychiatric comorbid disorders such as depression (Armenian, Morikawa, \& Melkonian, 2002; Kuo, Tang, \& Tsay, 2003). Although accounting for approximately $70 \%$ of war exposed groups (Sapir, 1993), the tendency to marginalize women in the study of mental health care provision highlights their potential for developing future dysfunctional psychosocial capacities (Foa, Keane, \& Friedman, 2000)

Published works that relate to the psychosocial implications of war exposures on Darfuri women and girls per se is scarce (Morgos, Worden, \& Gupta, 2008) but is crucial to effectively target the use of existing mental health provisions in the Sudan.

Ahfad University for Women (AUW) is a privately operated all women's university in Omdurman city, Khartoum state. With a core concern to educate, safeguard and protect the future of Sudanese women and girls (AUW mission statement, 1906), notwithstanding those who have been war-traumatized, AUW is home to approximately 209 Darfuri students, an average of $3 \%$ of the total AUW student population. In order to effectively translate this mission, psychosocial and mental health services should become part and parcel of university infra-structure for the relief or control of the progression of mental health illness among its internally displaced populations (IDPs). In preparing AUW war-trauma counsellors, appropriate personal and professional war-trauma counsellor training is required to qualify them in assuming a crucial responsible role in identifying the psychosocial needs among war survivors, especially as the trials and tribulations continue with an on-going Darfuri war that is further complicated by current ongoing post-displacement stressors (Badri, Crutzen, \& Van den Borne, 2012a).

\section{Evidence-based contextual outline}

In seeking to identify the psychosocial needs of Darfuri IDP war affected women, four interrelated research studies were conducted among AUW undergraduates. Through interpretative phenomenological analysis (IPA), the narratives of twenty Darfuri students demonstrated the harrowing effects of personal and family war exposures, witnessing warfare, and hearing about incidences within combat zones. Their stories illustrated an array of recurrent themes including, battles with heavy gun-fire, artillery attacks, and air raids, separation and loss of family members, injury, torture and death, 
kidnap, abduction and disappearance, and shortages of essential life sustaining supplies in internally displaced camps (Badri, Crutzen, \& Van den Borne, 2012a).

The severity of war exposures and symptoms for PTSD were assessed through the application of a culturally relevant version of the Harvard Trauma Questionnaire (HTQ, (Mollica et al., 1992), among 116 Darfuri AUW undergraduates. More than half reported being personally exposed as victims or as witnesses to war-related traumatic events with a strong association between direct war-related traumatic exposures and the full symptom of PTSD (Badri, Crutzen, \& Van den Borne, 2012b). Generalized anxiety and symptoms for major depression were measured according to the Hopkins Symptom Checklist-25 (HSCL-25, (Hesbacher, Rickels, Morris, Newman, \& Rosenfeld, 1980) where $56 \%$ and $51 \%$ manifested symptoms of anxiety and depression respectively. The most commonly endorsed anxiety symptoms were headaches, feeling fearful, and feeling restless and tense, while depression symptoms mostly reported were self-blame, feeling blue, and feeling no interest in usual activities. Anxiety scores were positively correlated with depression scores, and younger participants (15-20 years) who had a parent deceased were more anxious, while those whose father was a blue-collar worker (indicating low socio-economic status) were more depressed (Badri, Crutzen, \& Van den Borne, submitted).

Furthermore, evidence illustrated that they were confronted with a myriad of ongoing life hassles and urban-cultural challenges. Urban-cultural challenges and lack of environmental mastery occurred to most Darfuri participants as they relocated to Omdurman city which included negotiating an unfamiliar transport system, learning the routes and directions to important city landmarks, and insufficient funds for basic hygienic essentials.

Also, as a result of being physically distant from their families they lacked the shelter of parents, and lost familiar and rich social support networks such as the encouragement of extended family members. The associated emotional distress patterns that emerged were similar to symptoms of mood and anxiety disorders, according to the DSM IV criteria for symptoms of generalized anxiety disorder and major depression (Badri, Crutzen, \& Van den Borne, 2012b).

At a glance, there seems to be an emerging body of evidence to suggest the existence of a chain of events that in combination is associated with an increased vulnerability to psychosocial maladaptive symptoms. However, the reality is quite different. Darfuri participants resilience levels, measured by the Resilience Scale (Wagnild \& Young, 1993), illustrated that $57 \%$ of the 116 student participants were between moderate to moderately low levels of resilience (Badri, Crutzen, and Van den Borne submitted).

Coping resources and resilience characteristics were two primary aspects that influenced the mental health recovery of the Darfuri participants (Badri, Crutzen, and Van 
den Borne submitted). Strong religious practices and beliefs, availability of social support networks, reliance on making meaning (also known as meaning-attribution processes) and a positive future outlook seem to lend to their ability to cope with their subsequent emotional distress owing to war-related exposures, current on-going life hassles and urban-cultural challenges (Badri, Crutzen, \& Van den Borne, 2012a). The relationship between the component parts of mental health recovery (i.e., resilience levels, protective resources, and resilience characteristics) are addressed in an interactive processes to help identify the psychosocial needs of Sudanese affected by war in general and Darfuris in particular initiating a theory-based conceptual framework and thereby developing a successful trauma counsellor training program.

\section{Theory-based conceptual framework}

Operationalizing the totality of these Darfuri women's experiences leads to four unfolding concepts that relate to the evolving nature of the impact of location (war exposures), dislocation (displacement stressors) and relocation (coping). Concepts that incorporate these dynamic interactive processes of personal recovery are based on the theory of meaning-related processes (Folkman, 1997; Folkman, Moskowitz, Ozer, \& Park, 1997); resilience (Rutter, 1985; Masten, Best, \& Garmezy, 1990); and positive protective social support networks (Higgins, 1994; Werner, 1990).

The concept of meaning-related processes involves reappraising and attributing new meaning to unchangeable negative events that are usually beyond the control of the person (Lazarus \& Folkman, 1984), such as war-related traumatic exposures and post-displacement stressors and hardships. Moreover, religiosity typifies meaning attribution in that it provides a way of understanding suffering and loss (Wortman \& Silver, 1992). The evidence has shown that IDP Darfuri women have reappraised altered and replaced internal beliefs with new meanings to fit the new circumstances of their postdisplacement lives (Badri, Crutzen, \& Van den Borne, 2012a). They have also used religion in the form of prayer and reading the Quran in times of severe emotional distress owing to war-related exposures, current ongoing life hassles and urban-cultural challenges (Badri, Crutzen, \& Van den Borne, 2012a). Darfuris have attributed meaning that was congruent with cultural belief systems, personal goals, commitments, and motivation (Parker \& Folkman, 1997) by displaying their resilience characteristic of meaningfulness (Badri et al., submitted). Meaningfulness of life (Wagnild, 2008) has been used to describe the extent to which reappraised new situations help war-traumatized individuals deal with and offset the negative perceptions of war-related traumatic exposures and post-displacement stressors permitting realistic coping and a positive outcome in their adjustment process in the form of growth (Lerner \& Gignac, 1992). Operationally, Darfuris' meaningfulness resilience characteristics gradually allowed them to 
become aware of the urban-cultural clashes within their new environment at AUW and Omdurman city and placed new meanings based on their cultural upbringing that allowed them to interact with others in the community (Badri, Crutzen, \& Van den Borne, 2012a).

Resilience connotes inner strength, competence, optimism, flexibility, and the ability to cope effectively when faced with adversity (Johnson et al., 2009; Rutter, 1985). Resilience is operationalized by cultural norms and societal expectations (Luthar, Cicchetti, \& Becker, 2000), mobilized by internal motivation to eliminate stressful conditions, reconcile adverse experiences and achieve psychosocial adjustment in the face of adversity (Masten, Best, \& Garmezy, 1990). Evidence has shown that for many Darfuri female undergraduates, resilience seemed to be defined on the basis of meeting given societal expectations for behavioral competence (Elder, 1998), and others reflected the internal and external attributes of specific cultural norms (Luthar \& Cicchetti, 2000; Luthar, Cicchetti, \& Becker, 2000). They seemed to demonstrate resilience by generating opportunities for success within their immediate environments (Scarr \& McCartney, 1983; Werner \& Smith, 1992) whether these are positive self-perceptions and external competences (Luthar, 1991) or environmental mastery, or academic achievements (Greenberg, Lengua, Coie, \& Pinderhughes, 1999). Darfuri traditional and cultural upbringing demonstrates a disposition to enter functional roles early in life where children are expected to assume productive roles and responsibilities (for example young girls accept domestic responsibilities and taking care of younger siblings). This develops and fosters an adept self-image (Bronfenbrenner, 1979), social confidence (Garmezy \& Tellegen, 1984), prosocial behavior (Macksoud \& Aber, 1996), and self-efficacy (Ferren, 1999; Maslow, 1943). Operationally, it may be possible to infer that Darfuri women trajectories from adversity to recovery through previously learnt mechanisms of hardiness and resilience play a crucial role in overcoming their psychosocial distress.

Positive social supportive networks protect and buffer against adversity and severe distress by providing active reassurance, guidance, belonging, and affection. The evidence among Darfuri undergraduates seems to corroborate these concepts in the availability, range, frequency, accessibility and quality of close relationships and attachments they make in terms of alleviating psychosocial disorders (Henderson, Byrne, \& DuncanJones, 1981; Rutter, 1981; Schaefer, Coyne, \& Lazarus, 1981; Shapiro, 1995). Social support networks have created a crucial protective role in the post-displacement lives of Darfuri undergraduates such as in the absence of their customary social supports (parents, siblings, relatives, and neighbors). Darfuri women demonstrated a disposition to fall back on the habitual modes of protection from the unknown environment of Omdurman city and AUW by forming close interpersonal relationships with Darfuri students from the same tribe, ethnicity, and region, and belonging to the Darfuri student association at AUW (Badri, Crutzen, \& Van den Borne, 2012a). 
The interactive processes of the Darfuri IDP experience of war-related traumatic exposures and post-displacement stressors are important because in combination they seem to perpetuate the association with psychiatric disorder, specifically PTSD, anxiety and depression. However, the evidence provides pointers that their protective capacities of reappraising and attributing meaning to their new circumstances, capabilities in forming new secure and positive interpersonal relationships with other Darfuri students, resilient characteristics of meaningfulness, perseverance and self-reliance and moderate resilience levels amalgamate to expand their coping repertoire and highlights the interactive healing progression from psychosocial dysfunction to psychological growth in the face of multiple adversities (Badri, Crutzen, and Van den Borne submitted).

This interactive theoretical framework helps to explain the effective ways that Darfuri war-affected women cope with their adversities, and how Sudanese women operationalize trauma and recovery, which are both important concepts to consider when planning for a culturally appropriate and targeted war-trauma training program for Sudanese trauma counselor.

\section{Objectives for a sustainable Sudanese war-trauma counselor training program}

The first and foremost objective of Sudanese war-trauma counsellors is to serve waraffected Sudanese as their primary advocates for what is best needed in terms of resources utilization for the mitigation and prevention of milder mental health problems from becoming long-term mental health disorders. Furthermore, Sudanese war-trauma counsellors must be equipped with the ability to recognize the mental health consequences of war trauma and post-displacement stressors faced by on-going conflictaffected Sudanese, such as Darfuri women. Understanding issues in terms of culture, tradition and norms within each sector of Sudanese society is imperative to distinguish between the possible psychosocial responses and possible coping strategies employed. Sudanese war-trauma counsellors must also have the capacity to identify relevant social support systems and strengthen or enhance networks that will lend to the immediate assistance of needs and relief of war-related trauma symptoms such as PTSD, anxiety, and depression. Moreover, the aim of a training program is to professionally instruct war-trauma counselors through expert, clear, reliable and up-to-date information on culturally relevant concepts, methodologies and approaches that successfully recognize and relieve psychosocial stress, and appropriately facilitate and restore normality in the new location after displacement. Furthermore, training of Sudanese war-trauma counselors should include counselor qualities, awareness and skills consolidation that promote existing resilience levels, resilience characteristics and coping resources (Badri et 
al., submitted) as well as pave the way for active implementation of newly acquired coping and adaptive strategies (Papadopoulos, 2007) among Sudanese war-affected IDPs.

Based on the identification of Darfuri undergraduate women's war-related experiences and post displacement stressors, their psychosocial needs (Badri, Crutzen, \& Van den Borne, 2012a), and the interpretation of resilience theories as they relate to the Darfuri totality of experiences, the following are guidelines for the development of a comprehensive war-trauma counselor training program targeted to strengthen the efficacy of Sudanese mental health care providers in general, and build the professional capacity among AUW in-house counselors in particular.

Needs assessment is the catalyst that should propel war-trauma counselors' ability at identifying and knowing the specific Sudanese war-related psychosocial needs, vulnerabilities and risks. An AUW war-trauma counsellor should be well versed on the various norms, standards and traditions of a multicultural Sudanese society as she considers the totality of Darfuri student experiences: as an undergraduate student; as a war survivor; as young Darfuri women; pre-conflict aspects of Darfuri life and postdisplacement stressors of Omdurman life within a systemic approach (Papadopoulos, 2001; Papadopoulos, 2001; Papadopoulos \& Hildebrand, 1997). By teasing out the details which pertain to loss, in terms of identity, relationships, support systems, roles, obligations and usual routines the AUW war-trauma counselor identifies the loss of dignity and pride, decorum and morality, cultural and religious belief systems, that the Darfuri student/client has conceptualized and operationalized as needs in their current experiences of cultural incongruence, loss of familiar social support systems and networks, financial worries and academic challenges (Badri, Crutzen, \& Van den Borne, 2012a). Moreover, AUW war-trauma counselors should be able to recognize the symptoms that Darfuri student/clients are particularly unable to cope with, especially those factors relating to their pre- and post displacement stressors, the intensity of current stressors and war-related exposures and the duration of adversity (for example, factors of separation from family and culturally determined social support systems) which illustrates their vulnerabilities and risk factors for developing mental health problems (Badri, Crutzen, \& Van den Borne, 2012b).

Recognizing that during their on-going psychosocial stress of displacement, Darfuri female undergraduates' social cognition (Ostrom, 1984) informs their perception and interpretation of social information (Kinderman \& Bentall, 1997) which impacts their level of functioning (Badri, Crutzen, and Van den Borne submitted). Further, Darfuri undergraduates' social cognition and information processing may bear on their appraisal and reappraisal of stressors influencing their perceptions of social support with the belief that additional resources, in the form of significant others, are available (Badri, Crutzen, \& Van den Borne, 2012a). AUW war-trauma counsellors should be aware of Darfuri undergraduates' operational value and their use of social engagement (Runtz \& 
Schallow, 1997) as a positive function of seeking out the co-operation of others and as a coping mechanism against the effects of pre-and post war adversity (Badri, Crutzen, \& Van den Borne, 2012a).

Finally, identifying the socialization process and all that goes with it is highly influential and operational in Darfuri lives. For example recognizing the cultural norms and tradition that make up Sudanese gender-roles (Badri, 2008) which may inhibit Darfuri women from seeking trauma counseling is important to understand as they may act in increasing their risk and vulnerability to psychosocial dysfunction. An important pragmatic and contextual point is to implement a culturally relevant approach, for example, it is more likely that a Darfuri female student approaches and talks with other Darfuri women of the same ethnic tribe than with a man or other Sudanese woman.

Identification of resilience levels, resilience characteristics such as perseverance and meaningfulness, or as an aspect of the environment, such as coping social support resources are typical types of Darfuri resilience operating to protect and buffer against adversity (Badri et al., submitted). Improving on their healthy adaptive systems including: (a) fostering resilience levels, (b) enhancing the utilization of effective resilient characteristics (meaningfulness, perseverance and self-reliance), and (c) promoting the successful use of protective resources (social support networks) should be a focus among AUW war-trauma counselor training programs.

a. Fostering resilience levels aims to enable the Darfuri war-affected female student to conceptualize herself as a resilient individual. Recognizing her cultural and traditional context allows for an in-depth understanding of the issues faced and the facilitation of ownership of psychosocial responses. Helping students/clients with the skill of using words to describe emotions encourages the Darfuri student to become cognizant of how her exposure to war has impacted her mental health. Also by reinforcing habitual coping mechanisms, such as the use of prayer, their options will help mobilize resiliency and adaptation to new circumstances.

b. Enhancing the utilization of effective resilient characteristics encompasses an increased understanding of the mechanisms of resilience such as fostering and encouraging the pre-existing strengths. Being aware and discussing the factors/determinants of pre and post displacement stressors also helps emphasize positive qualities in the student/client by involving her to work out a solution together with the counselor; the counselor could say 'I can see that you are a great help to...', 'It was very brave of you to come all this way on your own', 'I know you are not feeling very strong right now, but you have shown a lot of strength'. Be specific, practical and realistic with advice; use phrases like 'would it be possible to...?', 'what might happen if...?', 'in what ways could you...?

c. Promoting the successful use of protective resources includes identifying and knowing the availability and accessibility of potential protective factors and social 
support groups that exist within the community. Those can be mobilized and deployed successfully according to the needs and situations among AUW war-affected female students in particular or within the larger population of IDPs in Umbada catchment of Omdurman city. Linkages can be developed with similar groups such as peer self-help support groups, mutual emotional support initiatives and creative activities which promote problem-sharing and community involvement, thereby reducing their isolation and vulnerability.

Recognizing and distinguishing between psychosocial disorders such as PTSD, anxiety, and depression accounts for the main aim of trauma focused therapies and treatment. AUW war-trauma counselors must be able to link between the traumatic event or adversity and the consequential emotions and beliefs systems. Equipped with specific training skills required for working with war-traumatized Darfuri women, AUW wartrauma counselors can challenge and modify the maladaptive behaviors of wartraumatized individuals (Thabet, Abed, \& Vostanis, 2002; Thabet \& Vostanis, 1999).

The requirements for an effective war trauma counselor is to be trained in the specialized methodologies, skills, and approaches that successfully alleviate psychosocial stress. The following recommendations of training in specific tools can be implemented within an AUW war-trauma counselor training program, but can also be transferable to other Sudanese war-trauma mental health professionals.

The effective use of screening instruments and mental health assessment tools as defined by the DSM IV diagnosis of PTSD, anxiety and depression which can adequately identify mental health priorities, understand the psychosocial needs, and detect psychiatric symptoms. The design and/or adaptation of screening tools, such as the Trauma Screening Questionnaire (Newman, 1976), to mirror local idioms of emotional distress that understands the impact of social structures and belief systems.

Narrative Exposure Therapy (NET) is a type of exposure therapy (Stapleton, Taylor, \& Asmundson, 2006; Taylor et al., 2003) that was developed to treat PTSD that resulted from prolonged, severe, and/or multiple exposures to war-related trauma. The efficacy of NET in under-developed countries has been demonstrated (Bichescu, Neuner, Schauer, \& Elbert, 2007; Neuner et al., 2008; Neuner, Schauer, Klaschik, Karunakara, \& Elbert, 2004; Verdeli et al., 2003) within different cultural settings (Neuner, Schauer, Elbert, \& Roth, 2002; P.L. Onyut et al., 2005; Schaal, Elbert, \& Nuener, 2009; Schauer et al., 2004) in the treatment of war-related PTSD and comorbid major depression symptoms in children, adolescents, and adults. Individual NET in combination with groupbased mourning comprises an effective treatment for traumatized war survivors who have lost loved ones and have symptoms of PTSD and depression and comorbid symptoms such as social withdrawal, low self-esteem, loss of trust, and feelings of guilt and shame (Neuner, Schauer, Klaschik, Karunakara, \& Elbert, 2004). NET, supportive counseling and psycho-education have been proven to have a positive effect with Sudanese 
refugees suffering from PTSD (Neuner, Schauer, Klaschik, Karunakara, \& Elbert, 2004). Guilt cognition reductions have also been demonstrated with the successful use of NET (Resick, Nishith, Weaver, Astin, \& Feuer, 2002; Marks, Lovell, Noshirvani, Livanou, \& Thrasher, 1998). Further the feasibility to train trauma counselors in NET has been demonstrated (Onyut et al., 2004). During a NET session the war-traumatized Darfuri will construct a detailed chronological account of her own biography in cooperation with the trauma counselor. The traumatic experiences would be documented by the trauma counselor to ensure trauma focus. The grief session allows the student/client to be exposed to feeling associated with the loss similar to the specific focus of guided mourning. At the end of the sessions, the Darfuri student should receive a written report of her autobiography. Each session lasts between 120-150 minutes for 4 weeks (Schaal, Elbert, \& Nuener, 2009).

Interpersonal therapy (IPT) is aimed at the treatment of major depression among conflict and post conflict victims in order to re-establish normal patterns of life as permanent long-term recovery goals (Klerman, Weissman, Rounsaville, \& Chevron, 1984). There is evidence that IPT may be effective in reducing symptoms of posttraumatic stress and depression, either delivered individually as single gender sessions (Mufson et al., 2004; Mufson, Weissman, Moreau, \& Garfinkel, 1999) or in a group format run in groups of 3-4 persons (Mufson, Gallagher, Pollack Dorta, \& Young, 2004). Restoration techniques which may can include normalization and validation of trauma-related symptoms and distress reactions to multiple losses, enabling help-seeking behaviors and empowerment. Darfuri students/clients participants within group IPT sessions for the treatment of depression and symptoms for PTSD should have the same or similar problem areas to enable appropriate goal setting, initiate therapeutic processes and foster positive group cohesion (Robertson, Rushton, Bartrum, \& Ray, 2004). Darfuri students that had lost parents and were coping with the grief, for example, may benefit from a discussion with other students' about their emotions and be open and willing to understand their own feelings. The feasibility to train trauma counselors in IPT has been demonstrated (Bolton et al., 2003).

Cognitive Behavioral Techniques (CBT) operates within the meaningfulness of social interactions and interpersonal environments (Baumeister \& Leary, 1995), that assimilate local and community customs. Those that examine the effects of war-related traumatic exposures within this context have been shown to optimize positive psychosocial outcome after traumatic exposures (Otto, Hinton, \& Korbly, 2003; Segal, Williams, \& Teasdale, 2002). Prayer, reading of the Quran and other religious practices have also been shown to reduce subsequent development of PTSD (Khouzam \& Kissmeyer, 1997) and can be viewed within the realm of relaxation techniques and vehicles to deliver CBT (Hinton, Pham, \& Minh, 2004). CBT that is offered to Darfuri undergraduate clients in a safe and understanding environment can produce psychosocial relief. In introducing 
CBT it may be important to reassure students/clients that its objective is not to change personal beliefs but to explore different ways of thinking about their experiences and their futures enabling them to develop new considerations.

Guided mourning for grief, although not explicitly measured among this group of Darfuris, is it highly likely that grief reactions compounded PTSD and depression. Culturally specific grief rituals need time to develop and are important for coping with loss. Intensive dialogue must be applied to ensure effective coping.

Psycho-education campaigns are important for reducing potential stigma associated with seeking and sustaining support. designed to support war-trauma survivors this instrument can be used in screening at-risk individuals by introducing basic understanding of mental health knowledge and providing accurate information about normal trauma responses and ways to cope (Howard \& Goelitz, 2004). Moreover, psychoeducation increases general awareness and disseminates information, particularly on the rationale and function of treatments and therapies such as CBT, NET and IPT and it is necessary that both the trauma counselor and student/client become active participants in this type of support to maintain motivation and persevere with treatment.

Psychological debriefing or Critical Incident Stress Debriefing (CISD) is a structured group program, which has been widely used in disaster counseling, predominantly with adults, with positive findings. It facilitates the discussion of fears, myths and beliefs, and promotes the discharge of feelings, and empowers war-traumatized individuals to build their futures (Thabet, Abed, \& Vostanis, 2002).

Group therapy has a higher client-counselor ratio which improves costeffectiveness. They promote effective catharsis, support, and a sense of identification with others, especially in bereavement, death and grief groups (Austin \& Godleski, 1999). In particular for women, widows' groups are very effective in organizing support and help for each other.

\section{War-trauma counselor qualities, awareness and skills consolidation}

Additionally a war-trauma counselor must possess the personal qualities and awareness that pertain to the sensitivity of the treatment as well as be an expert on the skills and practices for needs assessment. The war-trauma counselor can ask about ordinary daily life such as friends, leisure activities, who they live with, where they live, and then go on to talk about sensitive topic such as difficulties of present life, difficulties in the past experiences of violence, displacement worries, hopes and plans for the future.

War-trauma counselors must possess the personal qualities that create and foster trust and assure confidentiality, including establishing rapport, showing interest and responding to feelings of loss, loneliness and isolation (Inter-Agency Standing Committee, 2007). Skills consolidation, while similar to those for counselors in general, 
war-trauma counselors need to adhere to best practice protocols such as active listening, listening but not forcing talk, and mirroring. This is especially important because of the potential stigma associated with mental illness and barriers to health-seeking behaviors (Inter-Agency Standing Committee, 2007). Becoming aware of Darfuri's potential feelings of guilt, shame, hostility, anger and betrayal because of their experiences, war-trauma counselors should be empathetic, non-judgmental, showing respect and accepting. Finally, war-trauma counselors must be aware of how they conduct themselves when faced with very distressful stories. Self-observations, monitoring and examining own verbal and non-verbal communication and cues such as facial expressions, eye contact, body posture and movements and tone of voice significantly affect disclosures of very intense, sad and frightening thoughts and feelings as well as enable effective communication and facilitate the relaxation of students/clients.

\section{Discussion}

Sudanese culture, and in particular Darfuri tradition, continues to function within expected norms of gender roles, social commitments, and cultural expectations framed within an Islamic religious perspective, where social cohesion perceptions are interpreted. The experience of war-trauma and post-displacement stressors and the ensuing epistemologies of mental health can also be considered within this cultural conceptualization of social cognitions (Ostrom, 1984). Darfuri tradition and expected modes of behavior emphasize resilience levels, resilient characteristics, and external coping resources (Badri, Crutzen, and Van den Borne submitted) even with the most glaring of atrocities and the most severe of stressors among Sudanese survivors of war-trauma (Meffert \& Marmar, 2009; Stoll \& Johnson, 2007; Tipping, Bretherton, \& Kaplan, 2007). Extrapolating conceptualizations that are based on very different cultural presumptions would lose focus when considering the development of a Sudanese war-trauma counselor training program which promotes the psychosocial health of Darfuri women.

Attention has been given to the healing process unique to the war-affected Darfuri undergraduate female. Matching evidence-based experiential components of warrelated exposures and post displacement stressors with significant psychosocial distress and symptoms for PTSD, depression and anxiety has highlighted Darfuri women's ability to form protective social support networks, underline her moderate resilience levels, and exemplify her resilience characteristics shown as perseverance, self-reliance (Badri, Crutzen, and Van den Borne submitted). These factors are essential when designing and implementing a war-trauma counseling training program that successfully meets the psychosocial needs of war-traumatized Sudanese.

In April 2012, Ahfad Trauma Training Treatment Center (ATTTC), funded by a grant from the United States Department of the State, was the first nationally initiated 
project to promote war-related trauma counseling as a comprehensive, coordinated, and seamless mental health service delivery for Sudanese war survivors, including AUW students, and the Omdurman community (Umbada catchment area). It is envisaged that ATTTC will not only build on the capacities of AUW trauma counselors, but also transfer knowledge expertise and skills acquired to other organizations and institutions to carry similar roles within their communities throughout the nation.

The initiation of this war-trauma counselor training is intended to heal the emotional and social wounds of armed conflict and discourage helplessness, disempowerment and isolation among those who seek mental health. It is envisaged that the objectives, qualities and skills proposed within this manuscript will cater for the communal coping style that is characteristic of Sudanese culture.

Participants in the first training of war-trauma counselors included AUW staff in the school of Psychology, psychologists in the Military Hospital, and psychiatrists in private practice throughout Omdurman and Khartoum cities. The War Trauma Foundation (WTF) has provided the expertise needed to train this first batch of Sudanese wartrauma counselors based on their professional needs and working requirements. Although adept in the field of war traumatized survivors, the fifteen participants had little or no knowledge of specific concepts, methodologies and tools required for actual wartrauma counseling. Further, they had limited knowledge of personal characteristics required for effective war-trauma counselors in regards to qualities, awareness, and skills consolidation.

By implementing the afore mentioned training guidelines it is envisaged that AUW is provided with a best value-added position in meeting its vision and mission; educating, protecting and safe-guarding women as well as restoring effective resilience characteristics, promoting helpful interpersonal relationships and facilitating the development of new coping skills. Community mental health care providers and other educational institutions or organizations who work with war-traumatized Sudanese may also benefit from the implementation of these guidelines with regards to their helpfulness, capacity building and professional development in responding to the psychosocial needs of war-exposed Sudanese.

\section{Recommendations}

Religious, socioeconomic and other cultural influences all affect the acceptability of warrelated psychosocial training and interventions. The assessment of needs and the subsequent treatment of PTSD, anxiety, depression and other comorbid symptoms should not isolate individuals from their family or the community because this will impede recovery. Research is required to better understand the perceptions war-affected Sudanese have concerning mental health care, acceptability of care, willingness to continue 
with treatment, and ways to communicate with war affected Sudanese that validate their experiences as war survivors.

The challenge in each local situation is to validate assessment needs and adapt training guidelines that are relevant, understood and accepted by the war-trauma counselors and war-affected clients. For example introducing culturally sensitive approaches such as CBT, must be audited by the counselors within their context to ensure optimal benefit to their communities recovering from war-related exposures (Bryant \& Njenga, 2006). Furthermore, clear aids, flexible guidelines, and pragmatic manuals that successfully describe the needs of a group and predict the efficacy of treatment (Tarrier, Sommerfield, \& Pilgrim, 1999)

(Tarrier, Sommerfield, \& Pilgrim, 1999) will also facilitate the replication within other institutions, organizations and communities that require assistance in developing an effective war-trauma counselors training program. 



\section{Chapter 7 \\ General Discussion}

The specific needs of internally displaced persons should identify and guarantee their rights to protection and assistance during displacement, resettlement and reintegration.

Guiding Principles on Internal Displacement, United Nations, 2001 
The negative impact of war is apparent at various levels of the Sudanese society. Economic, social, and political instability is occurring on a large scale and the most vulnerable groups are women and children. Despite knowledge which has been gathered about African women, the mental health status of Darfuri women as internally displaced people (IDPs) outside the areas of conflict has not been documented. In view of this shortcoming, this thesis aimed to document women's experiences to the exposures of the ongoing armed conflict in Darfur, with an emphasis to investigate the forms, nature, and severity of their experiences as pertains to direct exposure as witnesses to armed conflict or hearing about violence in a situation of armed conflict. Furthermore, the studies have provided evidence for moderate to severe levels of post-traumatic stress disorder (PTSD), generalized anxiety disorder (GAD) and major depression disorder (MDD) symptomology. To further investigate the mental health status of female undergraduates, comparative studies addressed anxiety and depression symptoms between war-affected and non-war-affected young women. As a final point of interest, resilience levels and coping strategies were evaluated to explore resilience characteristics and coping resources as they relate to their psychosocial adjustment. To capture the need for developing a war-trauma counsellor training program in the Sudan, four interrelated studies among war-traumatized Darfuri internally displaced undergraduate women informs the basis that guides an outline for qualification development, capacity building and skills consolidation among Sudanese mental health care providers.

As a prelude to the consequential studies presented in this thesis, two theoretical frameworks were developed to capture the complexity of war-related traumatic exposures and post-displacement stressors of Darfuri female IDPs as well as to demonstrate the interactive connection between demographic socio-economic characteristics, psychological health and coping strategies in relation to providing an outline for psychosocial counselor training.

Based on the formulation of psychosocial assessments and therapeutic intervention design of the Australian Psychological Society's (Murray, Davidson, \& Schweitzer, 2008) and on the Stress Process theory (Beiser \& Hou, 2001; Beiser \& Hyman, 1997) an adapted framework that captured the influence of war-related traumatic exposures and post-displacement stressors on the psychosocial and psychological health of Darfuri IDPs was developed. The framework also took into account a set of predictive systemic variables to represent possible pathways that impact the coping strategies amongst Darfuri war-traumatized IDPs undergraduate female students (Model 1). 


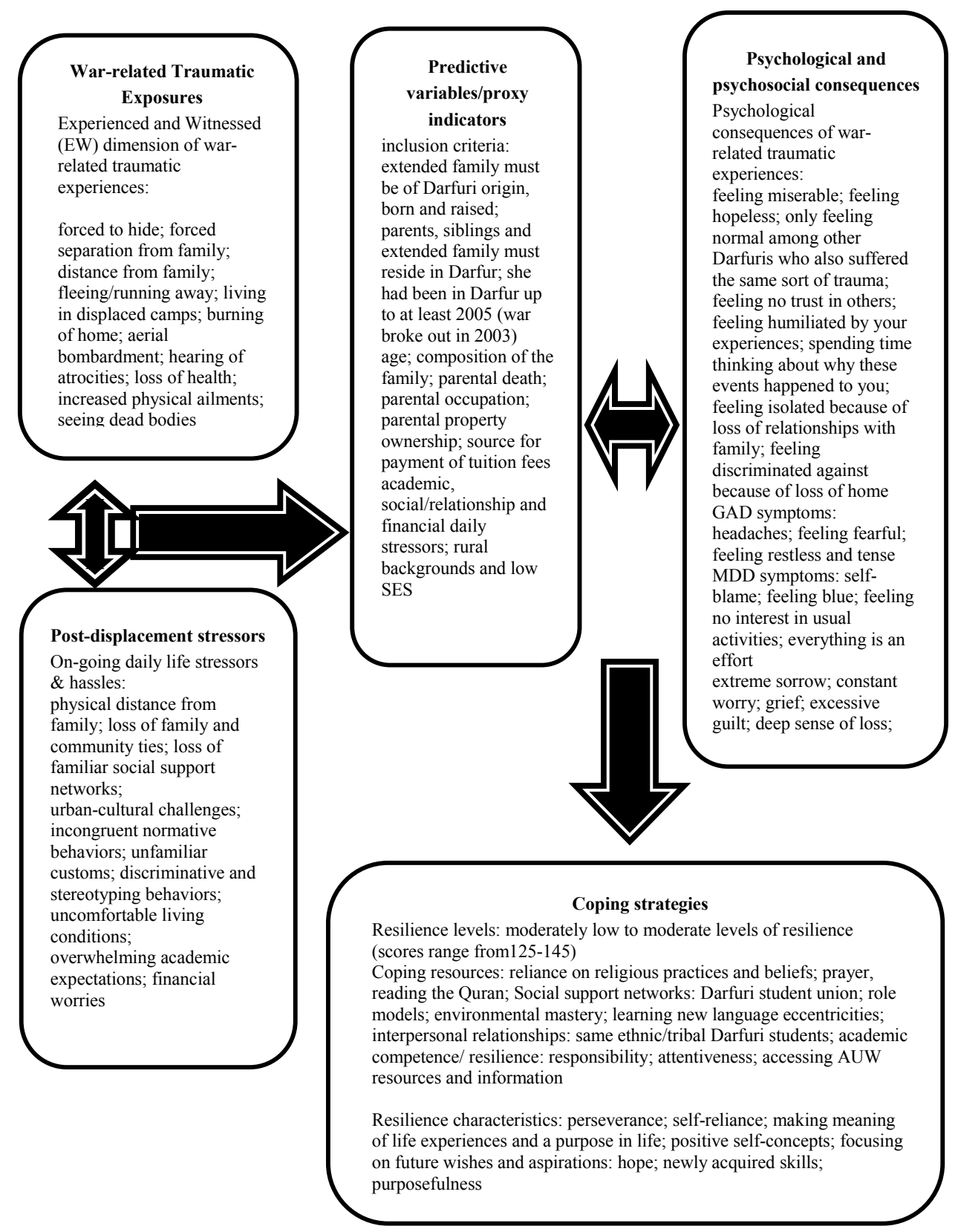

Model 1. Path representation of pre- and post-displacement stressors impacting on Darfuri IDP female undergraduates' psychological and psychosocial health

Furthermore, to indicate the degree of stress load and the relationship between stressors-mental health a transactional-sociological perspective (Katerndahl \& Parchman, 2002) is outlined in Model 2. Within this framework the impact of stressors pertaining 
to demographic socio-economic characteristics of the undergraduate students at AUW and their coping strategies are differentiated between two ethnically and experientially different populations: Darfuri war-traumatized IDPs and Omdurmani non-war-exposed undergraduate female students. An integrative adapted model was developed based on the Stress Process Model (Pearlin \& Schooler, 1978; Pearlin, 1989, 1999; Pearlin, Menaghan, Lieberman, \& Mullan, 1981) as well as incorporating advancing scientific understanding of the stress-mental health connection (Wheaton, 2010) differentiate the impact of stressors and coping strategies between two ethnically and experientially different populations: Darfuri war-traumatized IDPs and Omdurmani non-war-exposed undergraduate female students an integrative model was developed (Model 2).

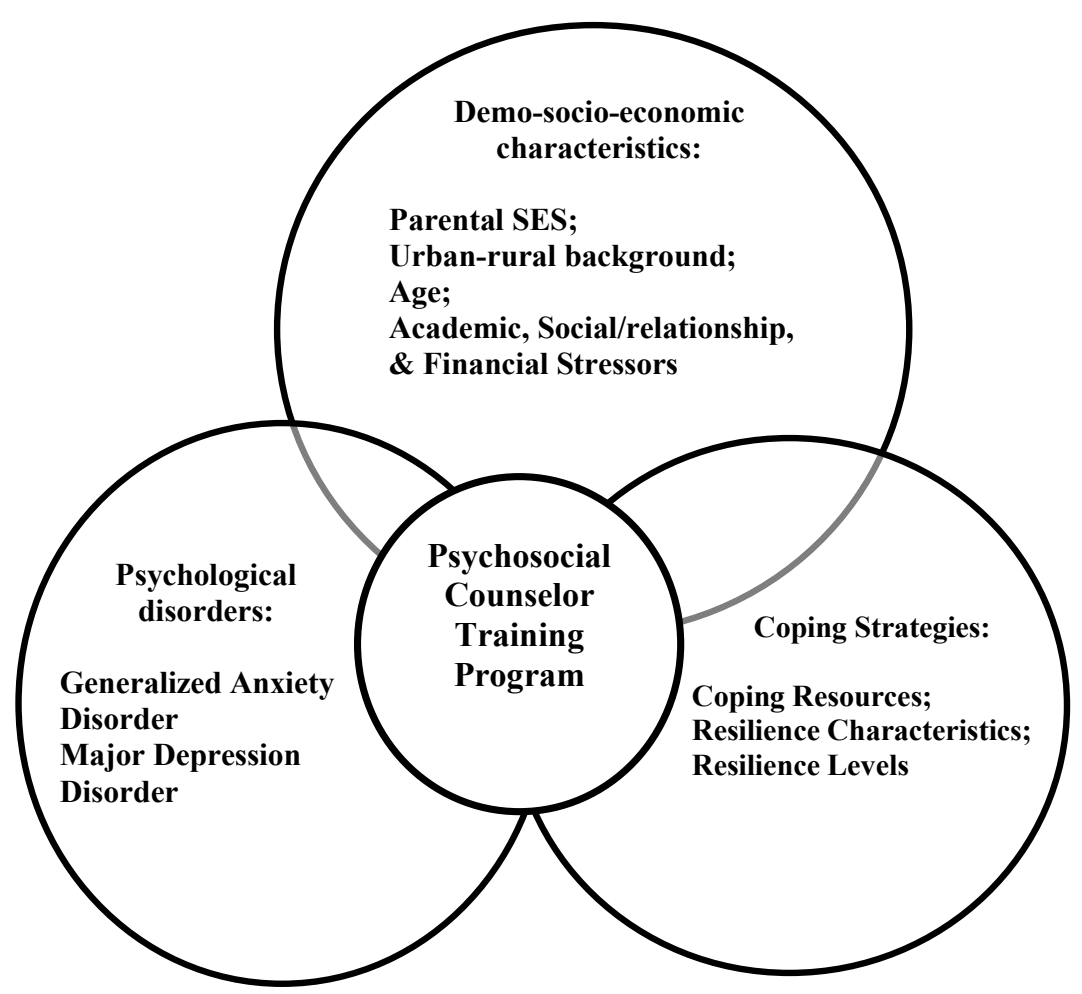

Model 2. An integrative model describing the impact of stressors and the influence coping resources, resilience levels, resilience characteristics in relation to developing a counselor training program

\subsection{Main Results}

A qualitative study among twenty Darfuri students personal accounts were collected in which the nature and severity of attacks perpetrated against the participants, family members, or friends in and around their villages in Darfur were clearly indicated. Fur- 
thermore, we identified war-related traumatic exposures, current on-going daily life hassles and stressors and urban-cultural challenges which increased the distress levels among Darfuri female university students (Badri, Crutzen, \& Van den Borne, 2012a).

Concerning the association between the range and severity of war-related exposures and the severity of PTSD among Darfuri female IDP undergraduates, the findings have chronicled that approximately two-thirds (67\%) of the 116 students have personally experienced and/or witnessed war-related traumatic events or have been in a combat situation (Badri, Crutzen, \& Van den Borne, 2012b). Also, Darfuri female IDP undergraduates exposed to traumatic war events were found to exhibit an $81 \%$ prevalence rate for PTSD (Badri, Crutzen, \& Van den Borne, 2012b). These findings are in line with those conducted within IDP camps in Darfur which found that both pre and postdisplacement conditions have predicted high incidences of traumatic reactions, mental and psychosocial dysfunction among women and girls (Kim, Torbay, \& Lawry, 2007; Morgos, Worden, \& Gupta, 2008; Rasmussen \& Annan, 2009; Rasmussen et al., 2010). Furthermore, other post-displacement stressors may include cultural dislocation (Eisenbruch, 1991), loss of normative social support networks and poor accommodation (Porter \& Haslam, 2005) which have also been suggested to increase psychosocial maladjustment as a direct interplay between the impact of war-related exposures and current on-going life stressors.

Struggling to find the means of travel to Omdurman city as they relocate in pursuance of their education, their confrontation with hassles related to relocating to $\mathrm{Om}$ durman city and their life at AUW, their academic challenges, urban-cultural clashes, and financial worries have all predicted a mental health burden on these young Darfuri women (Badri, Crutzen, \& Van den Borne, 2012a). Although the results show minimal variations between Darfuri and Omdurmani participants in terms of anxiety and depression scores, the data also shows that more Omdurmani urban, non-war-exposed undergraduates were depressed than Darfuris. This finding of a higher than expected prevalence of depression symptoms amongst Omdurmani may suggest a number of associated factors, such as highlighting the possibility of anticipatory anxiety symptoms (Thabet, Abed, \& Vostanis, 2002), as well as underscoring the prospect of the occurrence of daily life stressors (Rasmussen et al., 2010; Paardekooper, De Jong, \& Hermanns, 1999) amongst the Omdurmani undergraduates.

When comparing Darfuri war-traumatized rural undergraduate female students and Omdurmani non-war-traumatized urban undergraduates a set of possible predictive variables were taken into account to determine variations in GAD, MDD and resilience scores. While geographical background and place of origin (as indicators for urban-rural living), played a limited role in determining variations in anxiety and depression scores between Darfuris and Omdurmanis, a parent's death, source of payments of tuition fees, father's occupation, parents owning property in their place of origin (as in- 
dicators for SES), and age played a predictive role in mental health status, whereby younger participants and those who had one of their parents deceased were more anxious and those who were of a low socio-economic status were more depressed, indicating the possibility that the differences between Darfuris and Omdurmanis are less dramatic but more related to current stressful conditions of everyday life. The findings demonstrate that the pervasiveness of a low SES, including the potential impediment on parents to cover educational fees, costs, unemployed or blue collar workers, places a substantial burden on both groups of undergraduates to excel in order to justify the major financial sacrifices their families are making. Additional academic challenges, finance and budgeting problems, social stressors, accommodation adjustments and harsh living conditions (Badri, Crutzen, \& Van den Borne, 2012a) increase the risk for developing of anxiety disorders and depressive episodes (Vaz, Mbajiorgu, \& Acuda, 1998; Tomoda, Mori, Kimura, Takahashi, \& Kitamura, 2000).

Nonetheless, anxious and depressed Darfuri and Omdurmani undergraduates have demonstrated moderate to moderately low levels of resilience. Resilience has been linked to the personal qualities and predispositions of war traumatized Sudanese (Goodman, 2004; Tipping, Bretherton, \& Kaplan, 2007). The moderate to moderately low levels of resilience demonstrated by the Darfuri students reflect a pragmatic capacity to bounce back despite their adversity. Their cultural values and upbringing develops the potency for survival and fortitude given their harsh environmental surroundings (Young et al., 2005; Rasmussen \& Annan, 2009; Rasmussen et al., 2010), while purporting not to show its devastation. Also, being geographically distant from a war-torn Darfur and resettled in a more secure and stable environment (Omdurman city) may have served to increase their endurance and enhance their resilient expression, which relates to the significance of effective coping, endurance and resilience amongst waraffected Sudanese (Meffert \& Marmar, 2009; Simich, Hamilton, \& Baya, 2006; Stoll \& Johnson, 2007; Tipping, Bretherton, \& Kaplan, 2007). Furthermore, the ability to integrate many different coping strategies to protect and buffer against emotional distress has been shown with other Sudanese populations affected by war. The use of religious practices, forming meaningful interpersonal relationships, and showing signs of determination, perseverance and resilience has been evidenced (Goodman, 2004; Khawaja, White, Schweitzer, \& Greenslade, 2008; Schweitzer, Melville, Steel, \& Lacherez, 2006).

Darfuri students use religious practices and beliefs (praying and reading the Quran), form interpersonal relationships with other Darfuris, use social support networks, and a positive future outlook which seemed to lend to their ability to cope with war-related emotional distress, current on-going life hassles and urban-cultural challenges (Badri, Crutzen, \& Van den Borne, 2012a).

Darfuri students have also endorsed items relating to the avoidance subscale of PTSD symptomology such as feelings of detachment from others, an inability to feel 
emotions, and avoiding activities, and thoughts relating to the traumatic memory (Badri et al., Submitted). What may be happening is that Darfuri undergraduate female students are denying and suppressing their war-related traumatic experiences, as we have seen from their PTSD avoidance levels, which may manifest later in life. However, several researchers have argued that avoidance behaviours, thoughts, or feelings need not necessarily be a maladaptive responses; in fact it is considered to be effective in reducing the disturbing memory which is a therapeutic objective in many forms of psychological interventions (Sarraj, Punamaki, Salmi, \& Summerfield, 1996; Kagee, 2005). Their ability to avoid or numb their emotions and deal with their more immediate and current concerns of daily life stressors (Rasmussen, et al., 2010), such as financial worries, urban-cultural adaptation and academic challenges by relying on the various coping strategies (Badri, Crutzen, \& Van den Borne, 2012a) is reflected in their resilience score and seems not to have interfered with life as an undergraduate student.

Research has suggested that despite being of a low SES (Garmezy, 1991; Driscoll, 2006) with on-going life stressors (Wyman, Cowen, Work, \& Parker, 1991; Rew, TaylorSeehafer, \& Thomas, 2001) some undergraduate students seem to show unexpected high levels of resilience and perseverance. Similarly fifty-seven percent of anxious and depressed Darfuri and Omdurmani undergraduates have demonstrated moderate to moderately low levels of resilience despite their on-going stressors and low SES.

Additionally, familial and extra-familial support systems enhance resilience amongst low SES students (D'Imperio, Dubow, \& Ippolito, 2000; Christle, Harley, Nelson, \& Jones, 2007), while female students in many less developed countries confer that education improves resilient factors and coping capability (Dodani \& Zuberi, 2000). Evidence has suggested that both Darfuri and Omdurmani participants utilize their inner resilient characteristics (Wagnild, 2009) to raise their self-efficacy, self-esteem, giving them a greater sense of control over stressful situations (Harpham, 1994).

While acknowledging that the participants of the current study are all of Sudanese origin, and that clearly coping resources and resilience characteristics had an ameliorating effect on measures of anxiety and depression there are, nevertheless, striking differences between the 'extra-ordinary' major life stressors experienced by the Darfuri participants (war-affected) and those 'normal' tertiary stressors (non-war-affected) experienced by the Omdurmanis.

In the shadows of their war-related experiences and post-displacement stressors (Badri, Crutzen, \& Van den Borne, 2012a), Darfuris' resilience levels (Badri et al, submitted) demonstrate an ability of altering extreme emotional negativity to thinking positively by a process of continuous reappraisal and reinterpretation (Wortman, Battle and Lemkau, 1997). The ability to engage in 'making meaning' (Knight, 2006) as an internal resilient characteristic when faced with social/relationship, academic and financial stressors reflects a capability to address future difficulties more assertively. Further- 
more, the high endorsement of this resilience characteristic within the Darfuri group signifies an interaction that draws from their repertoire of spirituality, religious practice, sense of appreciation, gratitude and thankfulness of being alive (Badri, Crutzen, \& Van den Borne, 2012a) in an attempt to make meaning not only to the psychological effects of war exposure but also to the context of the experiences in their current lives indicating an urgency to adjust to university life, and an importance to successfully accomplish current goals; notwithstanding the pursuant of educational opportunity.

Despite high levels of anxiety and depressive symptoms (Badri, et al., submitted), and their endorsement of overwhelming challenges, such as cultural/ethnic incongruence, differences in language patterns, and navigating academic hurdles, financial worries and social/relationship stressors, Darfuri war-traumatized rural ethnic-minority undergraduates (Badri, Crutzen, and Van den Borne submitted), have shown a potential in increasing their psychosocial adjustment by utilizing effective coping resources. Much like other rural student communities (McIntire, Marion, \& Quaglia, 1990), Darfuris sought contact with others from the same ethnicity or tribal background (Badri, Crutzen, \& Van den Borne, 2012a). This protective buffer against psychological distress (Timmins \& Kaliszer, 2002) seems to emphasize the significance of social support networks and their interpersonal relationships with 'significant others' (Cope, 1972; Duru \& Poyrazli, 2007; Swift, 1988). Darfuri culture stresses the importance of group cohesion and positive ethnic identity similar to other ethnic minority communities (Holleran \& Waller, 2003; Werner, 1995). The premise is that social support networks buffer against an overwhelming number of stressors, especially those of new intake undergraduates, such as financial, academic and social/relationship (Badri, Crutzen, and Van den Borne submitted). Moreover, the integration of Darfuri coping resources and resilience characteristics tend to determine resilience levels and seem to affirm how they will cope with their new urban and student life at AUW (Badri, Crutzen, \& Van den Borne, 2012a; Badri, Crutzen, and Van den Borne submitted).

Resilience characteristics endorsed by the Omdurmani non-war-affected students that help them cope with social/relationship, academic, and financial stressors were mainly their ability to rely on themselves, equanimity, and existential aloneness. The group has also reported that largely due to the effects of a chronic disadvantaged environment, being categorized within a low socio-economic status, faced with family financial pressures, and a pervasive lack of resources (Badri, Crutzen, and Van den Borne submitted), they still have the capacity for self-reliance by taking on jobs optimizing their income, and spending wisely only on pertinent possessions, boosts competence and self-worth (Werner \& Smith, 1992).

Although the most salient familial and extra-familial protective coping resources may be a strong force in promoting resilience and encouragement (Ceja, 2004), they may themselves be compromised by a chronically disadvantaged environment, non- 
availability of economic opportunities and resources (Dubow, Edwards, \& Ippolito, 1997), and the debilitating effects of poverty may serve as an additive function to metal distress levels (Seidman et al., 1995; Seidman, Allen, Aber, Mitchell, \& Feinman, 1994), including depression (Assimeng, 1981; Badri, Crutzen, \& Van den Borne, submitted; Nagase et al., 2009), and may lose their protective impact (Wyman, Cowen, Work, \& Parker, 1991).

Academic stressors are eased by communicating and seeking information, guidance, and support from significant others, such as university staff, including counsellors. Their familiarity with environmental surroundings, people, and cultural mannerisms, functions as alleviators of daily life stressors by interacting with fellow Omdurmani students and pursuing leisure activities as a coping resource. Their active participation in extra-curricular activities including, AUW's team sports, choir and music bands as recreational activities suggests the multiple pathways to resilience (Masten, Best, \& Garmezy, 1990). Committing to these activities recognizes their resilience characteristic of equanimity, 'taking things in their stride', and 'taking things one day at a time', fosters a practice of commitment in future aspirations (Masten, Best, \& Garmezy, 1990).

On the other hand, Omdurmanis also indicated that they would rather be alone (i.e., 'existential aloneness') as a resilience characteristic and had lower needs for social relationships than Darfuri students. It is important to highlight the significance of this resilience characteristic as a 'coming home to yourself' (Wagnild, 2009), a preferred coping strategy to moderate Omdurmanis academic, social/relationship and financial stressors (Abela \& Hankin, 2009).

Our studies provide valuable insights reflecting the trends by which two distinctly diverse groups of undergraduates utilize coping resources and exhibit resilience characteristics in the face of academic, financial and social/relationship stressors. According to the results, Darfuris were more anxious in comparison with Omdurmani students when resilience characteristics were taken into account. Their minority representation as an ethnic-culture within a larger Omdurmani dominated ethnic-culture at AUW prompted them to identity with those who are ethno-culturally similar and hence endorse a high level of social support and solidarity. Also, their individual capacity for making meaning and meaningfulness as an inner resilience characteristic endorses their flexibility in reappraising stressful situations in the face of academic, financial and social/relationship stressors and challenges. Omdurmani students, more pragmatic, 'whatever it takes', tendency of self-reliance protects them against academic and financial stressors, as they feel they can 'handle', 'manage', and 'get through difficult times' on their own. Also, Omdurmanis were more likely to use friends and leisure activities as coping resources, and relied on a more urban-type equanimity and existential aloneness for social stressors.

In comparing between Darfuri and Omdurmani undergraduate students, whom both have a varied and distinct set of experiences, we have come to recognize that $\mathrm{Su}$ - 
danese undergraduate students have a reservoir of potential positive responses despite experiencing adversity, including war-related exposures and low SES.

Subsequently our initial impression that Darfuri students would have substantially higher levels of GAD and MDD due to their war-related traumatic exposures, their postdisplacement stressors, and their ethnic-minority and SES than their Omdurmani counterparts was laid to rest. Furthermore, our notion that Darfuris would fare far less in coping strategies and resilience levels was also proved inconsistent with the findings, as both groups utilized coping resources and resilience characteristics to achieve similar resilience levels (moderate-moderately low).

The implications of these findings suggests the importance of establishing a framework within which to develop an effective counseling service that identifies existing resilience characteristics and effective coping resources as those found within this sample of both Darfuri and Omdurmani students.

Not only should an effective counseling service take into consideration the totality of the Darfuri experience, and as such there may be a need to re-train trauma counselors in essential differential paradigms, including, re-conceptualizing traumatic reactions as reactions to adversity, rather than on the limiting conceptualization of PTSD, generalized anxiety disorder and major depression symptoms (Papadopoulos, 2002), but also to utilize appropriate cultural and psychosocial adjustment models which attribute meaning, equanimity and existential aloneness, as resilience characteristics, and focus on making meaning and having purpose, while also considering customary social support networks that provide protection and buffer against adversity (Masten \& Curtis, 2000; Masten, Tušak, Zalar, \& Ziherl, 2009; Matheny et al., 2002).

With these inferences in mind the goal of formulating a culturally sensitive wartrauma counselor training program must highlight aims that Sudanese counselors should also be equipped to cater for those who have not gone through war exposures. As such it is critical that to meet the high demand for Sudanese expertly trained personnel, qualification development guidelines should focus on capacity building, skills consolidation, psychosocial needs assessment tools, counselor characteristics, qualities and awareness training.

\subsection{Promoting Darfuri women's psychosocial health}

In an attempt to address the IDP situation in Darfur and Khartoum based camps, UN agencies and other international humanitarian groups (INGOs) put together evidencebased sustainable health development plans of action. These included assessing capacities, facilitating public-health and health promotion intervention initiatives. Although the Sudan government, under its Humanitarian Aid Community (HAC) has identified some key elements of these initiatives and some relating to the UN Guiding Principles 
for IDPs (OCHA, 1999), its lack of planned and consistent support to local psychological and psychosocial health promoters has caused a loss of the much needed social networks for continued intervention (OCHA, 2006). Research into IDP health needs including psychological and psychosocial health, have highlighted that community-based participation and culturally relevant treatment programs are essential requirements for effective psychosocial rehabilitation within IDP and refugee populations (Papadopoulos, 2002), including those in Omdurman city.

Consequently, four interrelated epidemiological studies have investigated the influence of war-related traumatic exposures and post-displacement stressors on the mental health status of IDP, ethnic minority, low SES, female university students, in relation to coping strategies and resilience levels.

Within the realms of Ahfad University for Women (AUW) mission, a psychosocial and mental health service is amongst its core concerns (AUW mission statement, 1906). AUW is home to approximately 209 Darfuri students, an average of $3 \%$ of the total AUW student population. In order to effectively translate this mission, and to substantiate the UN requirements for the provision of a community based psychosocial intervention program, AUW has picked up on the findings addressed in these four interrelated studies, as it sought to become the first nationally initiated project to the development of a war-trauma counselor training center as part and parcel of the university infrastructure.

In preparing war-trauma counselors, appropriate personal and professional wartrauma counselor training is required to qualify them in assuming a crucial responsible role in identifying the psychosocial needs among war survivors, especially as the trials and tribulations continue with an on-going Darfuri war that is further complicated by current on-going post-displacement stressors (Badri, Crutzen, \& Van den Borne, 2012a).

War-trauma counsellors must be equipped with the ability to recognize the mental health impact of war trauma, understand issues in terms of culture, tradition and norms within each sector of Sudanese society, identify the needs faced by war-affected Sudanese and distinguish between the possible psychosocial responses and symptoms of war-related trauma such as PTSD, anxiety, and depression. Moreover the coping strategies that ethnic minority university students utilize are important considering the negative attitudes that they hold toward seeking professional help (Leong, Wagner, \& Tata, 1995) and their underutilization of professional mental health care services (Daly, Jennings, Beckett, \& Leashore, 1995).

Based on the identification of Darfuri undergraduate women's war-related experiences and post displacement stressors and their psychosocial needs (Badri, Crutzen, \& Van den Borne, 2012a), AUW war-trauma counsellors should be well versed on the various norms, standards and traditions of a multicultural Sudanese society. According to Papadopoulos (2002), the totality of experience should be considered within a sys- 
temic approach, where details that pertain to loss, in terms of identity, relationships, support systems, roles, obligations and usual routines are identified as the loss of dignity and pride. The AUW war-trauma counsellors must also operationalize the Darfuri female students' decorum and morality, cultural and religious belief systems in the current experiences of cultural incongruence, loss of familiar social support systems and networks, financial worries and academic challenges (Badri, Crutzen, \& Van den Borne, 2012b). Moreover, AUW war-trauma counsellors should be able to recognize the symptoms that Darfuri student/clients are particularly unable to cope with, especially those factors relating to the intensity of current stressors and war-related exposures and the duration of adversity illustrating their vulnerabilities and increased risk for developing mental health problems (Badri, Crutzen, \& Van den Borne, 2012b). AUW war-trauma counsellors should also be aware of Darfuri undergraduates' use of social engagement (Runtz \& Schallow, 1997) as a positive function of seeking out the co-operation of others and as a coping mechanism against the effects of pre-and post war adversity (Badri, Crutzen, \& Van den Borne, 2012a). Finally, identifying the socialization process and all that goes with it is highly influential and operational in Darfuri lives. For example recognizing the cultural norms and tradition that make up Sudanese gender-roles (Badri, 2008) which may inhibit Darfuri women from seeking trauma counseling is important to understand as they may act in increasing their risk and vulnerability to psychosocial dysfunction. An important pragmatic and contextual point is to implement a culturally relevant approach, for example, it is more likely that a Darfuri female student approaches and talks with other Darfuri women of the same ethnic tribe than with a man or other Sudanese woman.

Recognizing the typical Darfuris' resilience characteristics and coping resources operating to protect and buffer against adversity (Badri et al., submitted) is imperative when offering counseling. Improving on their healthy adaptive systems including: (a) fostering resilience levels, (b) enhancing the utilization of effective resilient characteristics (meaningfulness, perseverance and self-reliance), and (c) promoting the successful use of protective resources (social support networks) should be a focus among AUW war-trauma counsellor training programs.

All manner of cultural influences affect the acceptability of psychosocial interventions and should not impede recovery or isolate Darfuris from accessing the trauma center. As such the challenge in each local situation is to validate assessment needs and adapt training guidelines that are relevant, understood and accepted by the war-trauma counselors and war-affected clients.

The evidence has shown that IDP Darfuri women have operationalized their resilience characteristics of meaningfulness and making meaning of their current lives by reappraising, altering and replacing internal beliefs with new meanings to fit the new circumstances of their post-displacement lives (Badri, Crutzen, \& Van den Borne, 
2012a). They have also used the coping resource of religion in the form of prayer and reading the Quran in times of severe emotional distress owing to war-related exposures, current ongoing life hassles and urban-cultural challenges (Badri, Crutzen, \& Van den Borne, 2012a). AUW war-trauma counselors must be aware of this meaningfulness resilience characteristic and coping resource among Darfuri undergraduates and promote its use as an effective tool which has allowed Darfuris to navigate urban-cultural clashes found within their new environment at AUW and Omdurman city (Badri, Crutzen, \& Van den Borne, 2012a).

Furthermore, for many Darfuri female undergraduates, resilience seemed to be demonstrated by generating opportunities for success within their immediate environments whether these are positive self-perceptions and external competences (Luthar, 1991) or environmental mastery, or academic achievements (Greenberg, Lengua, Coie, \& Pinderhughes, 1999). This may be a reflection of Darfuri traditional and cultural upbringing where specific cultural norms are adhered to early in life. For instance, young Darfuri girls are expected to assume productive roles and responsibilities such as accept domestic responsibilities and taking care of younger siblings, which develops and fosters an adept self-image (Bronfenbrenner, 1979), social confidence (Garmezy \& Tellegen, 1984), prosocial behavior (Macksoud \& Aber, 1996), and self-efficacy (Ferren, 1999; Maslow, 1943) through internal and external attitudes of societal expectations of behavior. Operationally, it may be possible to infer that Darfuri women trajectories from adversity to recovery through previously learnt mechanisms of hardiness and resilience play a crucial role in overcoming their psychosocial distress.

Social support networks have created a crucial protective role in the postdisplacement lives of IDP female undergraduates, such as in the absence of their customary social supports (parents, siblings, relatives, and neighbors). Darfuri women demonstrated a disposition to fall back on the habitual modes of protection from the unknown environment of Omdurman city and AUW by forming close interpersonal relationships with Darfuri students from the same tribe, ethnicity, and region, and belonging to the Darfuri student association at AUW (Badri, Crutzen, \& Van den Borne, 2012a).

The interactive processes of the Darfuri IDP experience of war-related traumatic exposures and post-displacement stressors are important because in combination they seem to perpetuate the association with psychiatric disorder, specifically PTSD, anxiety and depression. However, the evidence provides pointers that their protective capacities of reappraising and attributing meaning to their new circumstances, capabilities in forming new secure and positive interpersonal relationships with other Darfuri students, resilient characteristics of meaningfulness, perseverance and self-reliance and moderate resilience levels amalgamate to expand their coping repertoire and highlights the interactive healing progression from psychosocial dysfunction to psychological 
growth in the face of multiple adversities (Badri, Crutzen, and Van den Borne submitted).

\subsection{General remarks of key findings:}

Studies of different war-traumatized groups have contributed to a patchwork of knowledge necessary for recognizing the impact of war-trauma and the possible psychosocial consequences. However, it is not possible to ascertain the complete picture of those who have experienced war without embedding a deeper understanding within a sociocultural composition in the midst of generating an effective psychosocial intervention program.

To this end the key findings of these studies highlight the fact that Darfuri undergraduates' present mental health is decidedly influenced by their current on-going daily life hassles and stressors and urban-cultural challenges, the most pertinent of which included academic, social/relationship and financial stressors. Recognizing the daily life hassles of academic life, the loss of familiar social support networks, and lack of money for even the basic of needs, adapting and living in the different cultural environment of Omdurman city seem to contribute significantly to their increased levels of distress.

Furthermore, it may be possible to call attention to the possibility that under extreme stressor exposure (both pre- and post-displacement), certain resilience characteristics and resilience levels may have a diminished impact on the prevalence of mental health status, as can be seen by their moderate-moderately low resilience levels despite calling on existing social support networks.

The contribution that is specifically made by these studies not only adds to the existing body of knowledge on coping with war but also establishes the importance of entrenching the studies within a cultural socioeconomic framework that best describes all the influencing factors of adversity, and places PTSD, GAD, MDD and other comorbid symptoms within a culturally relevant needs assessment framework for the subsequent treatment of war-affected Sudanese.

The research approach following a sequential design of studies from qualitative to quantitative was a choice based on a desire to achieve as thorough as possible a picture of the Darfuri female IDP population. As such the studies have provided baseline information on war-related trauma exposure, symptom levels, correlations between symptom variables of PTSD, GAD and MDD, as well as the different demographic socioeconomic background variables otherwise non-existent among this war-affected group of Sudanese. 


\subsection{Study limitations}

With the lack of published research amongst this Sudanese community, the focus on women in this dissertation stems from the view that they have been traditionally marginalized in studies of mental health epidemiology and in-depth investigations of mental health care needs. The studies describes in this dissertation, have addressed this important empirical gap in relation to substantiating the mental health status as well as resilience levels of Sudanese female undergraduate students.

Firmly establishing the studies within the Sudanese context; socially, culturally, and economically, the study outcomes underscores a prevailing 'sameness' among both waraffected and non-war-affected female undergraduate populations, whereby all students display a similar burden of mental health problems.

The cumulative stressor exposure and chronic stress experienced by both groups of undergraduates have significantly improved the generalisability of mental health status among university-aged women in that, regardless of ethno-socio-economic background, all students' feel the brunt of the current Sudanese despondency and despair.

The studies presented in this dissertation have notably added to the resilience literature within the Sudanese context. They call attention to the possibility that even under conditions of on-going civil war, poverty, and a general breakdown of law and order, both Darfuri and Omdurmani female students portray a veneer of resilience. It is an encouraging finding that although symptom levels of GAD and MDD are prevalent; resilience has consistently emerged as the most common outcome trajectory.

Finally, the study outcomes have prompted the development of a targeted psychosocial counselor training program for university-aged women, as well as informing the Sudanese mental health care providers on the totality of psychosocial needs among Darfuri war-affected female undergraduates.

\section{Participants}

The study of a unique and specific type of undergraduate may in fact be its pitfall. The fact that we studied just one sector of the general population (university students) and one gender in one private university may limit the generalisability of the results. Although the current sample of Darfuri undergraduates may have a somewhat 'advantageous' status in that they have been given the opportunity to leave Darfur in times of war and become university students, denotes a certain non-representation of the wider population of girls whose schooling has been interrupted as a result of being within the war zone or within an IDP camp; they still seem to exhibit seriously high levels of PTSD, GAD and MDD.

Within the limitations imposed by issues of access to case-study sites, the study's small sample size and exclusivity of a single educational institute may have resulted in a 
fairly homogenous group; AUW undergraduates as opposed to female undergraduates from other universities, and may have limited generalizations to other female Sudanese affected by war, such as southern Sudanese. The logistical challenges faced by the principle researcher were mainly due to failed numerous attempts to penetrate the gates of other Sudanese universities who have Darfuri war-affected populations. Despite the principle researchers' insistence and perseverance to gain access to these Khartoumbased universities, doors were continuously closed due to the current socio-political situation in the country.

Nevertheless, our sample comprised of fairly diverse ethnic groups among the Darfuri society, and the data has provided evidence regarding an association between warrelated traumatic events and PTSD. However, it is envisioned that with a larger sample of Darfuri female undergraduates and further analysis should be undertaken before any firm conclusions are established for the quantification of prevalence rates of psychological distress, in particular PTSD, GAD and MDD.

Clearly the exclusivity of investigating a single female sector of the Sudanese society curbs the generalisability for mental health status, coping strategies and resilience levels with the general public. However, targeting university-aged women was the sole focus of the present studies due to the lack of published research amongst this group of Sudanese women thereby addressing this empirical gap as well as offering speculative inferences in identifying demo-socio-economic variables to the mental health outcomes of, university-aged Sudanese women.

\section{The choice of design}

This explorative and descriptive study included the limitations inherent to the use of a cross-sectional design; such as the difficultly to separate cause from effect between the included variables Longitudinal research designs are needed, especially to determine whether coping resources and resilience characteristics influence the subsequent development of anxiety and depressive symptoms and stressors, or whether the onset of symptoms and stressors negatively impacts the types of coping strategies undergraduates employ. The differences between Darfuri and Omdurmani participants in terms of coping resources and resilience strategies, however, are independent of the crosssectional design.

\subsection{The way forward}

Further research is needed to broaden and deepen the explorative nature of investigation carried out in these studies. With that in mind, it could be possible to examine whether the current associations between the triple burden of war: war traumas, post- 
displacement stressors and psychosocial morbidity can be replicated in other university samples, including undergraduate men.

Furthermore, longitudinal studies of health consequences of armed conflict, though face additional logistical and methodological challenges, have demonstrated their efficacy in identifying a more profound understanding of the lived lives of both wartraumatized and non-war-traumatized individuals (Lie, 2002).

There is evidence to suggest that psychosocial interventions aimed at ameliorating PTSD amongst survivors of war-related trauma is well placed and can be applied in preventive, rehabilitative and reconstructive spheres. Psycho-education, skills training, and relief activities such as games, arts and sports are some ways shown to have significance with Bosnian youth (Betancourt, 2005). Participatory approaches in the form of mentored community development activities, skills building and teaching appropriate coping strategies have been suggested to facilitate the reintegration of Eritrean youth (Farwell, 2001). Research among Sudanese refugees in Canada seem to suggest a process of cultural adjustment whereby psychological and social resources are met based on traditional coping strategies and customary social support networks (Simich, Este, \& Hamilton, 2010). Furthermore, Meffert and Marmar (2009) found a culturally informed version of Interpersonal Therapy (IPT) to be effective in the treatment of PTSD and depression among Darfuri refugees in Cairo (Meffert \& Marmar, 2009). Further research is needed to answer the question of which combination of multi-level approaches can be successfully integrated as a model of psycho-social intervention to best provide support and counseling amongst this population of Darfuri female students. A culturally appropriate multi-level approach can be designed and implemented by AUW.

Trained trauma counselors providing a buffer against the stigmatization of PTSD and mental health may also serve to supervise peer and social support groups counseling sessions that are aimed at ameliorating the memory of war-related traumatic events. Furthermore, evidence-based insight is required into the perceptions waraffected Sudanese have concerning mental health care, acceptability of care, willingness to continue with treatment, and ways to communicate with war affected Sudanese that validate their experiences as war survivors.

The challenge in each local situation is to validate assessment needs and adapt training guidelines that are relevant, understood and accepted by the war-trauma counselors and war-affected clients. For example introducing culturally sensitive approaches such as CBT, must be audited by the counselors within their context to ensure optimal benefit to their communities recovering from war-related exposures (Bryant \& Njenga, 2006). Furthermore, clear aids, flexible guidelines, and pragmatic manuals that successfully describe the needs of a group and predict the efficacy of treatment (Tarrier, Sommerfield, \& Pilgrim, 1999) will also facilitate the replication within other institutions, 
organizations and communities that require assistance in developing an effective wartrauma counselors training program.

Finally, accurately conceptualizing psychological symptoms and resilience amongst undergraduates has not been authenticated and on the other the instruments used to measure depression, anxiety, and resilience have not been culturally validated within this Sudanese context. This requires further research.

\subsection{Concluding remarks}

This study provides valuable insights reflecting the trends by which two distinctly diverse groups of students utilize coping resources and exhibit resilience characteristics in the face of academic, financial and social/relationship stressors. According to the results, Darfuris were more anxious in comparison with Omdurmani students when resilience characteristics were taken into account. Their minority representation as a ethnicculture within a larger Omdurmani dominated ethnic-culture at AUW prompted them to identify with those who are ethno-culturally similar and hence endorse a high level of social support and solidarity. Also, their individual capacity for meaning as an inner resilience characteristic endorses their flexibility in the face of academic, financial and social/relationship stressors and challenges. Omdurmani students, more pragmatic, 'whatever it takes', tendency of self reliance protects them against academic and financial stressors, as they feel they can 'handle', 'manage', and 'get through difficult times' on their own. Also, Omdurmanis were more likely to use friends and leisure activities as coping resources, and relied on a more urban-type equanimity and existential aloneness for social stressors. Recognizing that undergraduate students have a reservoir of potential positive responses despite experiencing adversity, including war-related exposures and low SES, suggests the importance of establishing a framework within which to develop an effective counseling service that identifies existing resilience characteristics and effective coping resources as those found within this sample of Darfuri and Omdurmani students. The implications for trauma counselors may be a need to re-train in essential differential paradigms, including, re-conceptualizing traumatic reactions as reactions to adversity, rather than on the limiting conceptualization of PTSD, generalized anxiety disorder and major depression symptoms; attributing meaning, equanimity and existential aloneness, as resilience characteristics; disempowering helplessness in the therapeutic relationship; and focusing on the eudemonic (meaning and purpose) of well-being (Papadopoulos, 2004). 


\section{Summary}

Civil war in the Sudan has produced up to ten million internally displaced people (IDPs) and refugees since the late 1950s. More recently, Darfur has seen its fair share of devastation on its population and infrastructure, with an average IDP population of 2.5 million (World Health Organisation, 2008). Heavy gun-fire, artillery attacks, and air raids, separation and loss of family members, injury, torture and death, kidnap, and shortages of essential life sustaining supplies occur in regularity (Hamid \& Musa, 2010; Kim, Torbay, \& Lawry, 2007; Morgos, Worden, \& Gupta, 2008; Rasmussen \& Annan, 2009).

The frequency of serious mental disorder, such as post-traumatic stress disorder (PTSD) and major depression disorder (MDD) (APA, 1999) has been investigated in projects worldwide among those who have experienced the devastation and trauma of armed conflict, indicating that even with the passage of time, these two mental disorders showed persistently high levels of symptoms (Kinzie et al., 1990; Mollica, Donelan, Tor, \& al., 1993; Steel, Silove, Phan, and Bauman, 2002). Likewise studies carried out in low-income countries have identified a strong association between PTSD symptoms, generalized anxiety disorder (GAD) and MDD (De Jong et al., 2001; Tang \& Fox, 2001). Furthermore the influence of post-displacement stressors demonstrated its effects in terms of symptoms of GAD, MDD, and other related mental disorders (Lee, Lee, Chun, Lee, \& Yoon, 2001; Silove, Steel, McGorry, \& Mohan, 1998).

This study was designed to shed light on the untold story of sufferings among Darfuri women IDPs. The intention of this project was to give a psycho-socio-cultural dimension to the severe problems faced by a group of female Darfuri undergraduates. Interviews were performed and subsequently epidemiological measurements of warrelated traumatic exposures, post-displacement stressors, and war-trauma symptoms including PTSD, GAD, and MMD were carried out. We expected to find high levels of these war trauma symptoms, despite the passage of time. Additionally, postdisplacement stressors were expected to influence mental health status by increasing distress levels.

The study also aimed to gain a broader and more differentiated overview of the psychological health status among war-traumatized and non-war-traumatized female undergraduates. In so doing, Darfuri and Omdurmani female undergraduates studying at Ahfad University for Women (AUW) were compared along GAD and MDD measures. Additionally these two ethnically and experientially different groups were compared on resilience levels, resilience characteristics and coping resources that help them deal 
with daily academic, social/interpersonal, and financial stressors. We expected to find that Darfuri students would have higher mental health problems than their Omdurmani sisters and consequently Omdurmanis would show higher resilient scores. We also predicted that each group would utilize different coping resources and demonstrate dissimilar resilience characteristics.

In light of the anticipated hypotheses, the project would conclude by proposing a tailor-made mental health care provision within AUW in-house counseling office to cater for the psychosocial needs of its Darfuri internally displaced female undergraduate population.

The importance of context in the understanding of a Sudanese community's psychosocial sequelae to war-related traumatic exposures and post-displacement stressors confronts us with the ethical and professional challenge of delivering a well-informed and targeted mental health care provision. There is a need among Sudanese mental health care professionals for consolidation of characteristics, qualities, and awareness that pertain to more knowledge and counseling skills in the treatment of war-affected IDPs, especially young women.

The study reported in Chapter 2 unveiled the personal accounts of twenty Darfuri undergraduates using a semi-structured interview protocol. The Darfuri students' stories illustrated that they were exposed to an array of traumatic war events, including fleeing/running away, forced to hide, forced separation from family, distance from family, living in displaced camps, burning of home, aerial bombardment, and seeing dead bodies. Also discussed during the interviews were their current on-going life challenges, emotional distresses, and coping strategies. The Darfuri students' stories illustrated that they were confronted with myriad current life hassles and urban-cultural challenges, and had high levels of emotional distress as they relocated to Omdurman city. Strong religious practices and beliefs (praying and reading the Quran), ability to form interpersonal relationships, availability of social support networks, and a positive future outlook seemed to lend to their ability to cope with their subsequent emotional distress, including extreme sorrow, grief, mourning, and constant worry owing to war-related exposures, current on-going life hassles and urban-cultural challenges.

Apparently the psychosocial effects of being exposed to armed warfare seem to be etched deeply in the memories of all twenty Darfuri IDP female undergraduates despite the noticeable passage of time. To capture the intensity and severity of war-related traumatic experiences and its association with post-traumatic stress disorder on a wider scale of Darfuri IDP female an exploratory cross-sectional study among a representative sample of Darfuri female university students at AUW ( $\mathrm{N}=123$ ) was the purpose of study presented in Chapter 3. Using an adapted version of the Harvard Trauma Questionnaire (HTQ), war-related exposures and post-traumatic stress disorder (PTSD) symptoms were assessed in February 2010. Means and standard deviations illustrated 
the experiential severity of war exposure dimensions and PTSD symptom sub-scales, while Pearson correlations tested for the strength of association between dimensions of war exposures and PTSD symptom sub-scales. Approximately $42 \%$ of the Darfuri participants reported being displaced and participants have experienced an average of 28 war-related traumatic events either as victims or as witnesses $(S D=14.24$, range $0-40$ events). Also, there was a strong association between the experiential dimension of war-related trauma exposures and the full symptom of PTSD. Moreover, the refugeespecific self-perception of functioning sub-scale within the PTSD measurement scored a mean of $3.2(\mathrm{SD}=.56)$, well above the 2.0 cut-off.

Even though the investigation has yielded similar results, if not higher, than those of previous studies among war-affected Sudanese, in terms of the association between traumatic war-related exposures and symptom rates of PTSD, a broader and more differentiated overview of the psychological health status among Sudanese female undergraduates was initiated with the expectation that war trauma and post-displacement stressors would yield higher psychological distress than those who have not experienced war and displacement. As such Chapter 4 presented the results of a crosssectional study in which Darfuri war-traumatized and Omdurmani non-wartraumatized female undergraduates $(\mathrm{N}=299)$ were assessed and compared in terms of generalized anxiety disorder (GAD), major depression disorder (MDD) (Hopkins Checklist HSCL-25; Hesbacher, Rickels, Morris, Newman, \& Rosenfeld, 1980) and resilience scores (Resilience Scale; Wagnild \& Young, 1993). Ethno-socio-eco- demographic variables were used as a set of proxy indicators to distinguish between the two groups experientially, ethnically, and economically. The data showed the $56 \%$ of the Darfuri sample manifested symptoms of GAD and 51\% had MDD. Similarly 54\% of the Omdurmani undergraduates were anxious and 58\% depressed. The most commonly endorsed anxiety symptoms amongst both Darfuri and Omdurmani groups were headaches (84\%), feeling fearful (80\%), feeling restless and tense (73\%), while depression symptoms mostly reported were self-blame (79\%), feeling blue (78\%), feeling no interest in usual activities (72\%), and everything is an effort (89\%). Fifty-seven percent of the total sample indicated a moderate to moderately low level of resilience, with very little variance between Darfuri and Omdurmani mean scores (5.3 and 5.1, respectively). Anxiety scores were positively correlated with depression scores $(r=.68, p<.001)$, but they were not correlated with resilience scores $(r=-.09, p=.08)$. Depression scores, however, were negatively correlated with resilience scores $(r=-.19, \mathrm{p}<.001)$.

Chapter 5 described and compared resilience levels, resilience characteristics and coping resources that both buffer and protect against daily academic, social/interpersonal, and financial stressors among these two ethnically and experientially different groups: Darfuri war-traumatized and Omdurmani non-war-traumatized female undergraduates. Implementing both internationally-used and culturally- 
grounded assessment tools the findings of a cross-sectional study for 116 Darfuri and 299 Omdurmani undergraduate students was presented to determine the student's habitual use of external resources and internal resilient characteristics. An eight item checklist was developed to include external coping resources, such as family, friends from the same ethnicity, recreation and hobbies, and five internal resilience characteristics like self-reliance, perseverance, meaning, and equanimity to manage academic, financial and social/relationship stressors. Darfuris were more likely to use contact with and support from people of the same tribe and ethnicity as coping resource, while Omdurmanis were more likely to use friends and leisure activities as coping resources. Regarding internal resilience characteristics, the Darfuris relied more on attribution of meaning to cope with stressors in general. Darfuris were more anxious in comparison with Omdurmanis when resilience characteristics were taken into account. The Omdurmanis relied more on themselves for academic and financial/economical stressors and on equanimity and existential aloneness for social stressors. Each group of students used different external coping resources and possessed different resilience characteristics with regards to managing academic, financial and social/relationship stressors. Inhouse mental health services can benefit from this study when designing counselor training or intervention programs with regards to encouraging undergraduates to use their effective resilience characteristics and coping resources in their attempts to adapt and manage stressors.

Equipped with the knowledge of the totality of Darfuri IDP experience; both warrelated exposures, post-displacement stressors and psychosocial sequelae, Chapter 6 provides guidelines for qualification development, capacity building and skills consolidation of the existing Sudanese mental health care. In line with this objective and based on four previous interrelated studies among war-traumatized Darfuri internally displaced undergraduate women, the current study provides a contextual and conceptual framework that outlines explicit war-related psychosocial needs assessment tools, specific war-related trauma counselor training and recommends particular counselor characteristics, qualities, skills consolidation, and awareness that pertain to strengthening the efficacy of Sudanese trauma counselors with regards to their helpfulness in responding to the needs of special groups such as war-affected Darfuri women.

Based on the research presented in this dissertation, some general conclusions can be drawn on promoting the psychosocial health of war-affected Darfuri females. Firstly, for the most part our results replicated the studies made in other parts of the world where devastation is experienced by those exposed to war-related events (Paardekooper, De Jong, \& Hermanns, 1999; Papadopoulos, 2002; Peltzer, 1999; Rogers \& Leydesdorff, 2004) as well as the wide range of severe symptoms of psychological distress (Karunakara et al., 2004; Roberts, Damunda, Lomoro, \& Sondrop, 2009; Roberts, Ocaka, Browne, Oyok, \& Sondrop, 2009). Furthermore an association between PTSD 
symptoms and war-traumatic exposures was strongly indicated amongst these Darfuri IDP women.

Contrary to popular belief, and our own expectations, very little differences were found in term of GAD and MDD scores amongst Darfuri war-traumatized and Omdurmani nonwar-traumatized female undergraduates. These very similar levels of psychological disorder may be accounted for by the chronicity of low socio-economic status (SES) regardless of the predictive variables for urban-rural living ethnic diversities of Darfuris and Omdurmanis. The elimination of socio-eco-demographic barriers only seems to highlight that post-displacement stressors are more relevant in the explanation of psychosocial distress among Darfuri students (Adewuya, Ola, Olutayo, Mapayi, \& Oginni, 2006; Joyce, 2010; Sharma, Parnian, \& Spielberger, 1983), while the possibility of anticipatory anxiety symptoms are more pertinent amongst the Omdurmani undergraduates (Thabet, Abed, \& Vostanis, 2002).

Also contrary to our expectations that Omdurmanis would show higher resilient levels than Darfuris, since the striking differences between the 'extra-ordinary' major life stressors experienced by the Darfuri participants (war-affected) and those 'normal' tertiary stressors (non-war-affected) experienced by the Omdurmanis. However we can conclude that both Darfuri war-traumatized and Omdurmani non-war-traumatized groups' resilience levels were the same. The prospect of associated factors such as the occurrence of daily life stressors amongst these undergraduate female students as a whole, might explain their shared experiences to current on-going daily stressors and reflecting a common cultural pragmatic capacity to bounce back from adverse conditions of everyday life in a least developed country (Lundberg, Cantor-Graae, Rukundo, Ashaba, \& Östergren, 2009; Mumford, Minhas, Akhtar, \& et. al., 2000).

As predicted resilience characteristics and coping resources to deal with daily academic, social/interpersonal, and financial stressors were very differently manifested. Darfuri undergraduates predominantly utilized religious practices and beliefs (praying and reading the Quran), formed interpersonal relationships with other Darfuris, used social support networks, and had a positive future outlook which collectively seemed to lend to their ability to cope with war-related emotional distress, current on-going life hassles and urban-cultural challenges, especially in terms of daily academic, social/interpersonal, and financial stressors. Furthermore, their ability to engage in a process of continuous reappraisal and reinterpretation (Wortman, Battle, \& Lemkau, 1997) of their current lives signifies an interaction that draws from their repertoire of spirituality, religious practice, sense of appreciation, gratitude and thankfulness of being alive in an attempt to make meaning not only of the psychological effect of war exposure but also the context of the experience in their current lives indicating an urgency to adjust to university life, and an importance to successfully accomplish current goals; notwithstanding the pursuant of educational opportunity. 
Omdurmani undergraduates reported that faced with family financial pressures, the effects of a chronic disadvantaged environment, a low socio-economic status, and a pervasive lack of resources enabled them to met these challenges by being self reliant, such as taking on a job and optimizing their income, equanimity, and existential aloneness all shown to boost competence and self-worth among the urban poor (Werner \& Smith, 1992).

Furthermore, the assessment of needs and the subsequent treatment of PTSD, anxiety, depression and other comorbid symptoms should be embedded in contextual and conceptual frameworks relevant to the totality of experiences among war-affected groups, which have been consistently indicated by longitudinal studies(Constantine, Chen, \& Ceesay, 1997; Tomoda, Mori, Kimura, Takahashi, \& Kitamura, 2000). There seems to be a need for integrated rehabilitation efforts where socioeconomic influences and other cultural factors are essential in the promotion of psychosocial and mental health among war-affected groups.

Finally, to ensure the efficacy of psychosocial interventions for Sudanese waraffected groups, the war-trauma counselors must audit the tools and approaches undertaken to ensure contextual relevancy within their communities (Bryant \& Njenga, 2006). As such, the description of psychosocial needs among Darfuri women recovering from war-related exposures, the assessment tools, therapeutic approached such as a culturally sensitive cognitive behavioral therapy (CBT), counselor characteristics, qualities, skills and awareness that are outlined in this dissertations' guidelines are embedded in empirical cultural context pertinent to secure optimal benefit. The replication of these predictive variables, methodologies and conceptual frameworks are recommended as aids to verify the specific psychosocial needs, skills training, and efficacy among Sudanese war-trauma counselors' treatment approaches within their institutions, organizations and communities (Tarrier, Sommerfield, \& Pilgrim, 1999). 


\title{
Arabic Summary
}

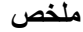

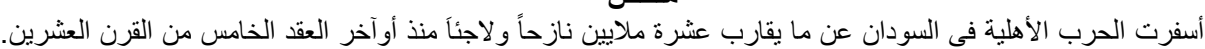

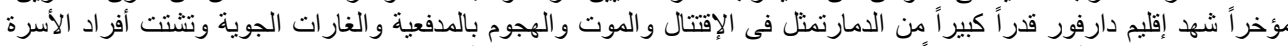

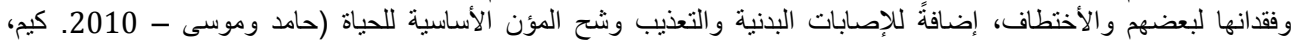

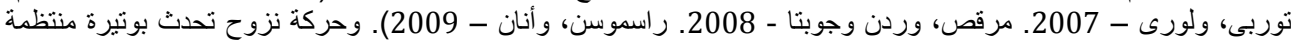 \\ وسط سكانه وحتى بلغ عدد النازحين 2007 مليون نسمة (منظمة الصحة العالمية - 2008).

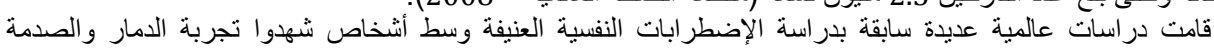

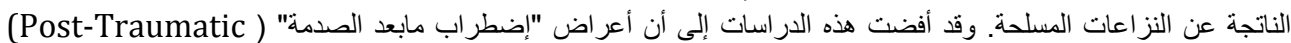

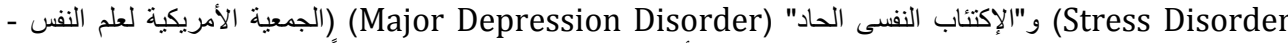

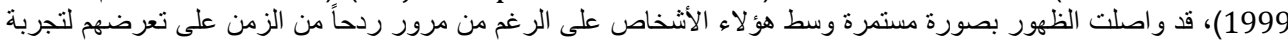

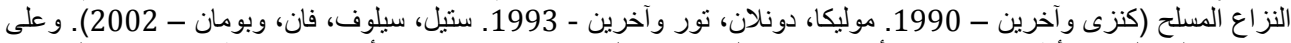

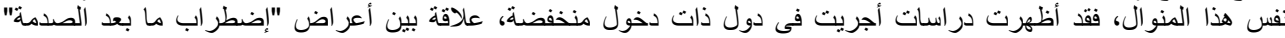

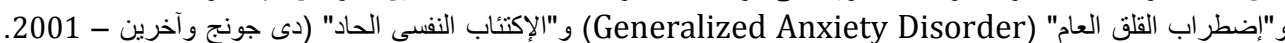
تانج وفوكس - 2001). يضاف إلى ما سبق أن الضغوط النفسية الناتجة في مرحلة ما بعد النزوح (Post-displacement)

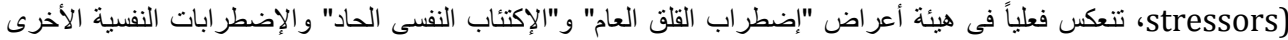

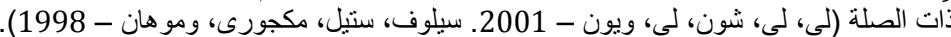

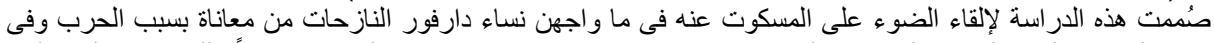

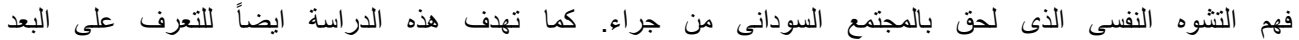

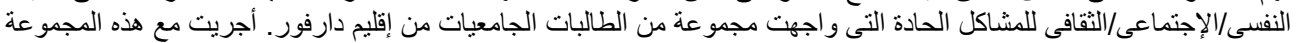

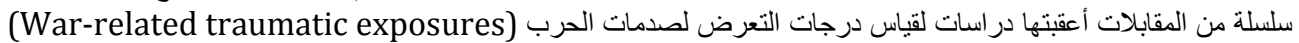

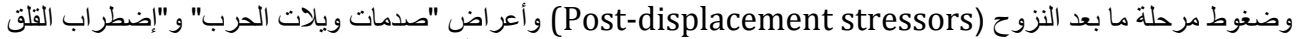

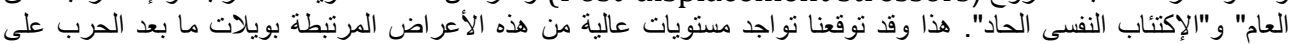

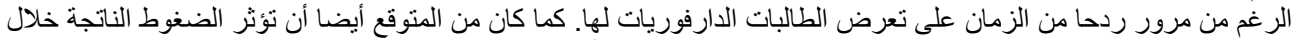

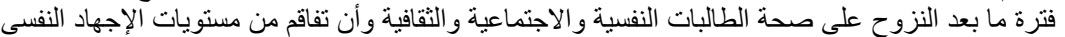

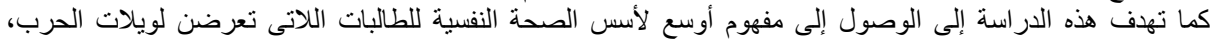

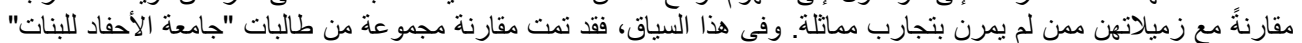

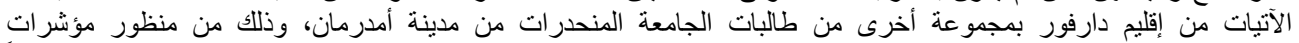

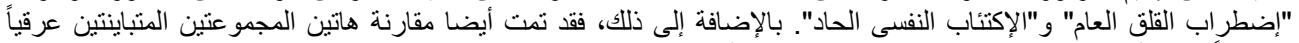

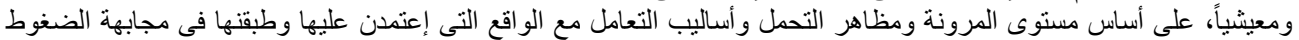

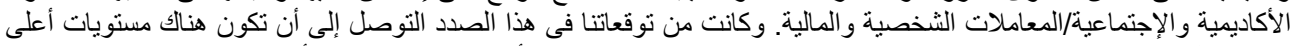

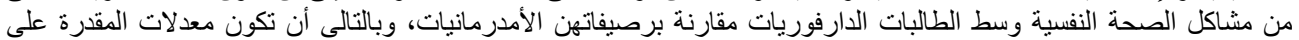

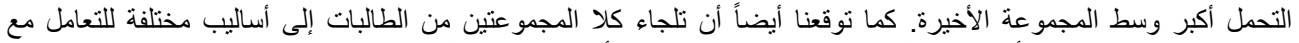

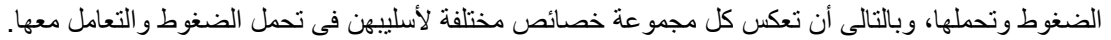

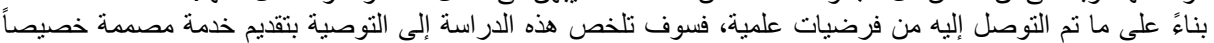

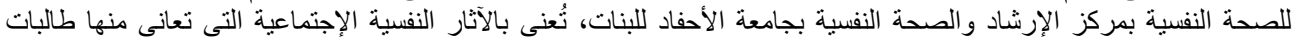
الجامعة النازحات من إقليم دارفور.

ركز البحث المنشور في الفصل الفصل الثانى من الرسالة على التجارب الثخصية التى مرت بهات بها عشرين طالبة جامعية من إقليم

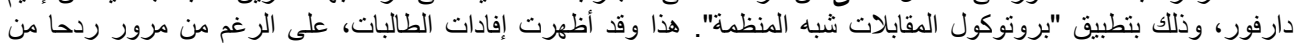

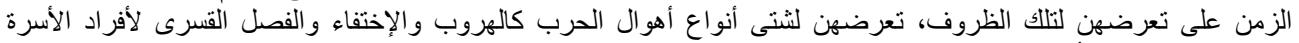

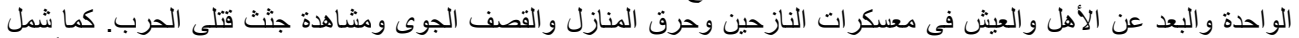

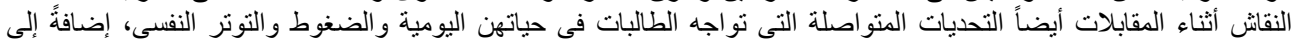

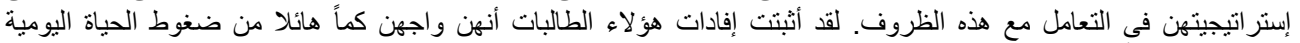

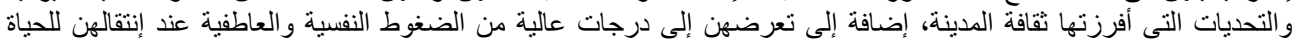

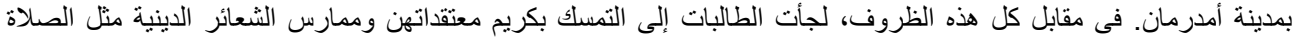




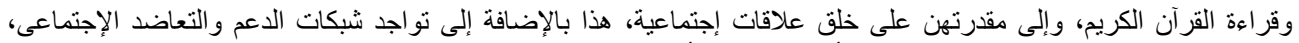

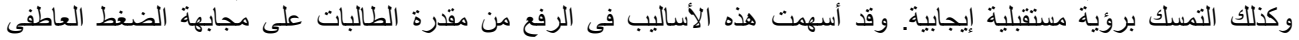

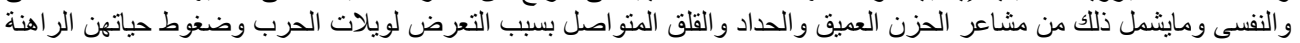
وتحديات الحياة الحضرية.

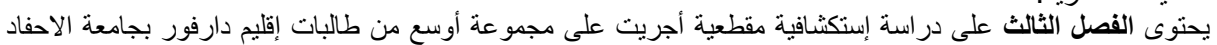

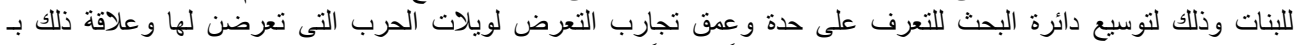

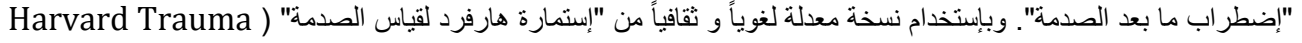

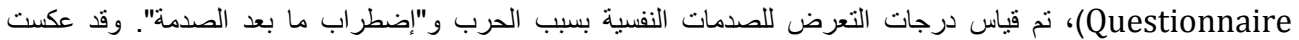

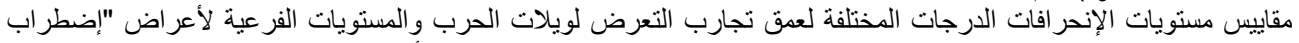

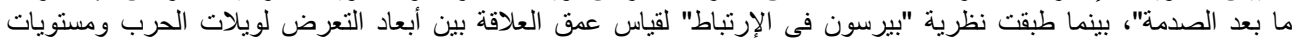

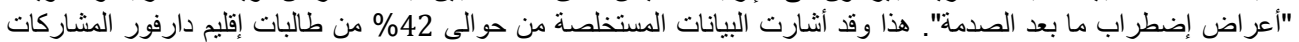

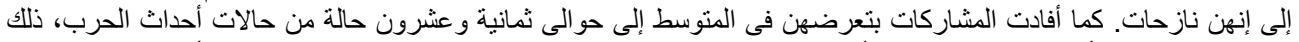

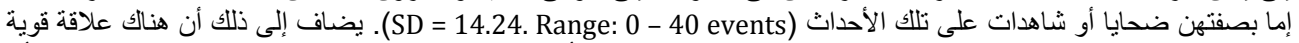

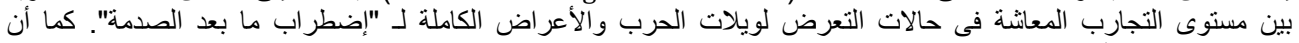

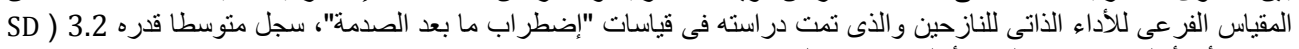

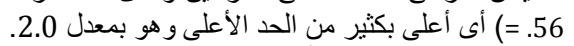

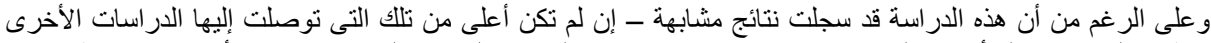

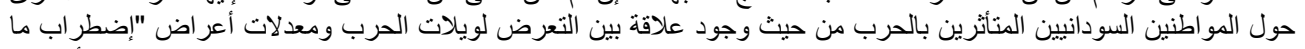

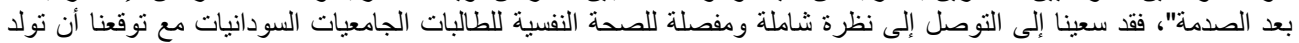

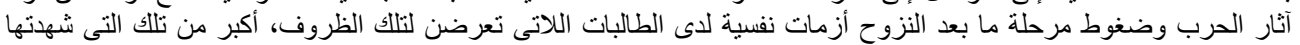

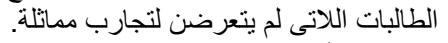

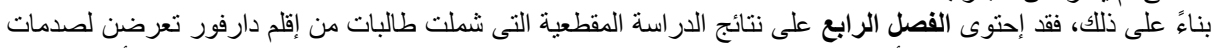

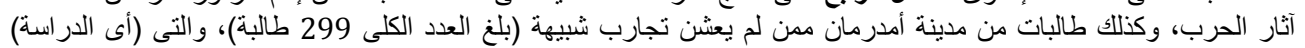

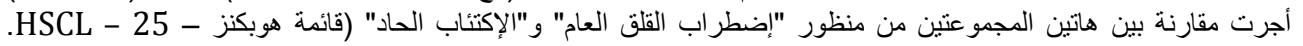

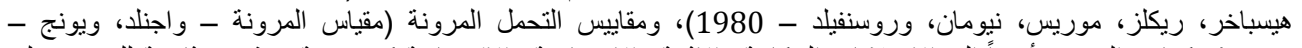

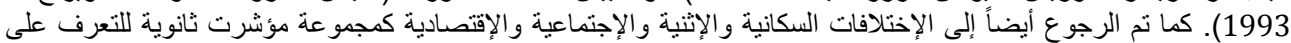

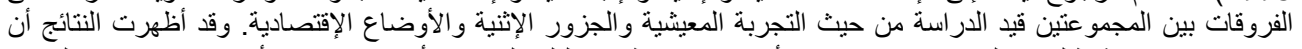

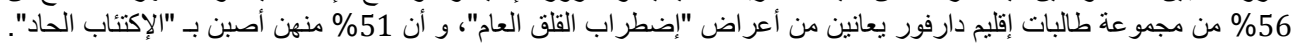

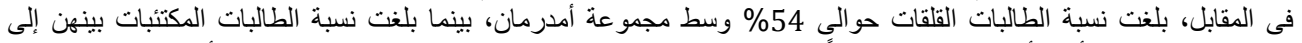

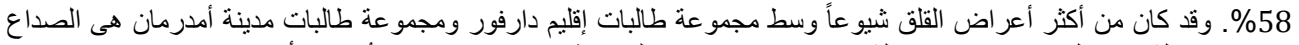

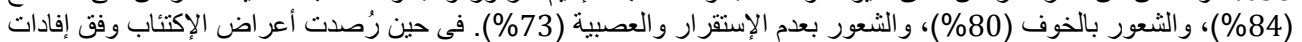

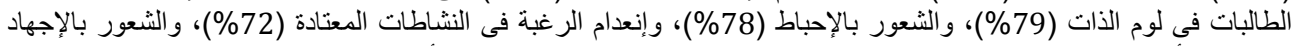

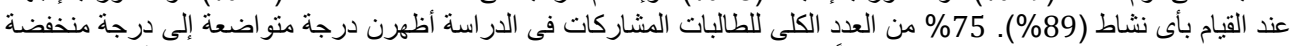

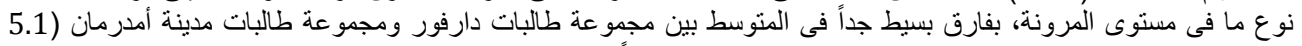

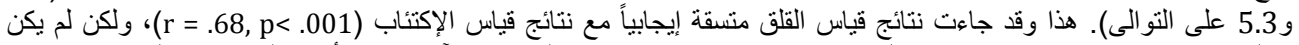

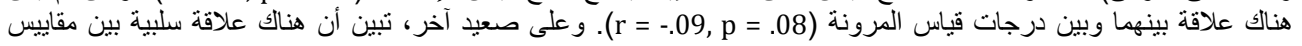

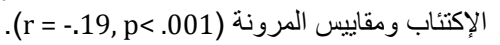

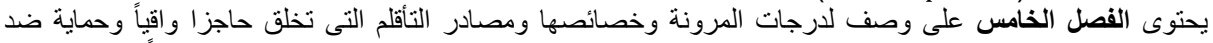

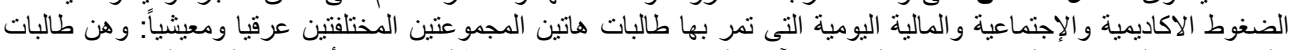

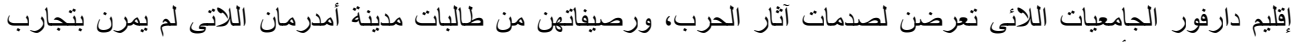

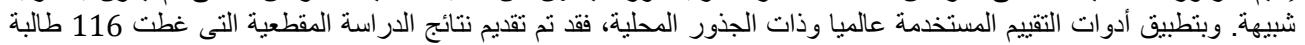

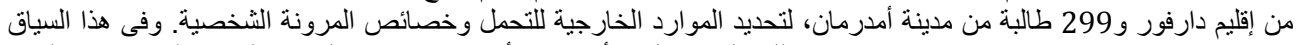

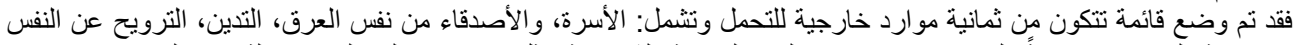

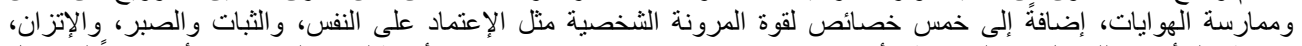

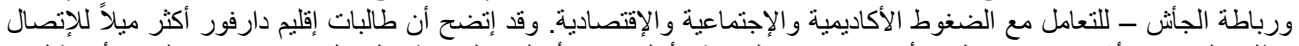

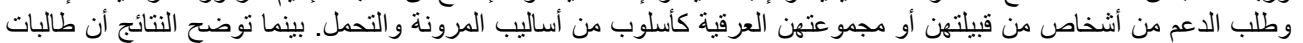

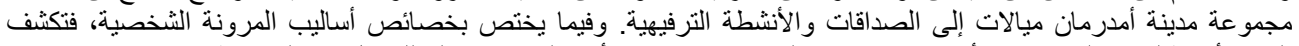

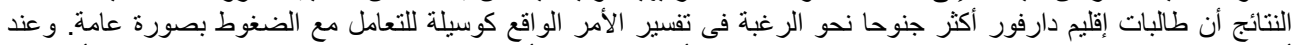

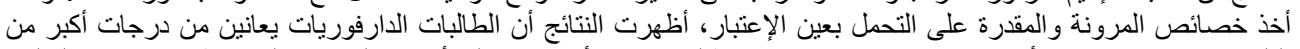

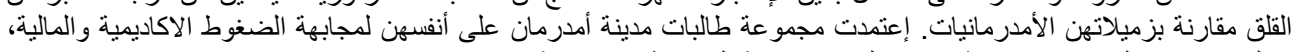

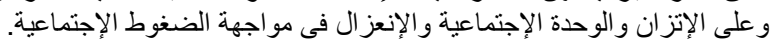

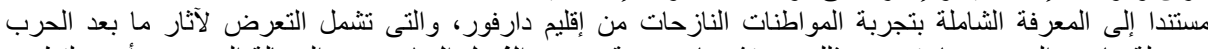

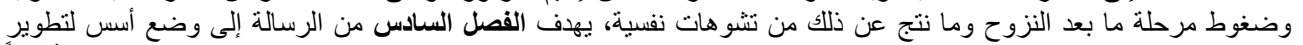

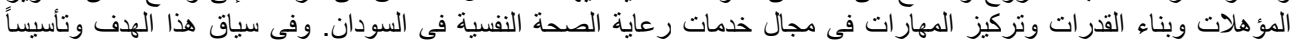




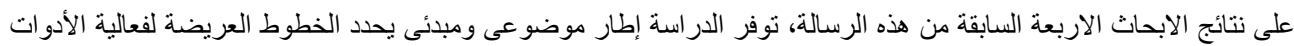

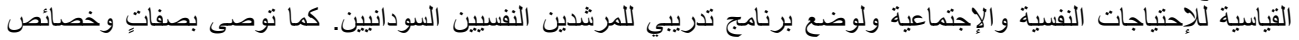

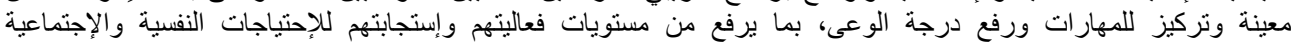

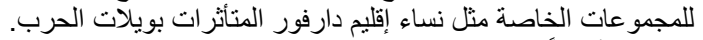

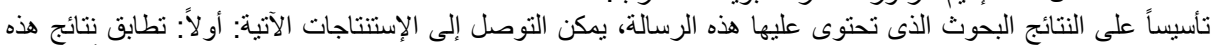

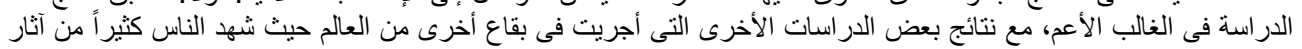

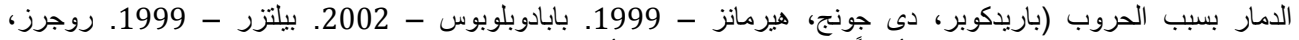

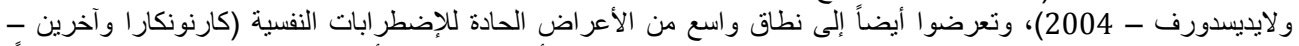

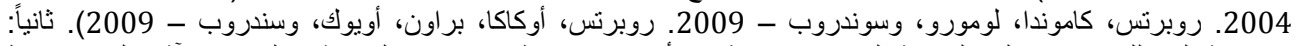

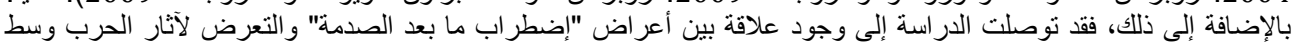

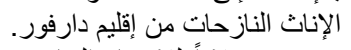

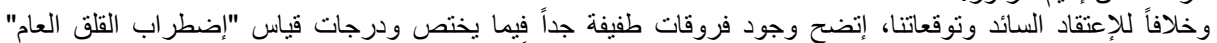

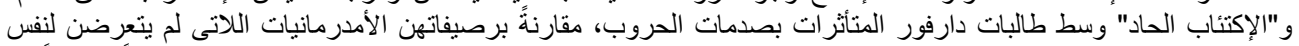

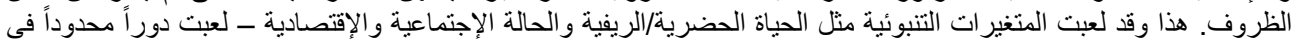

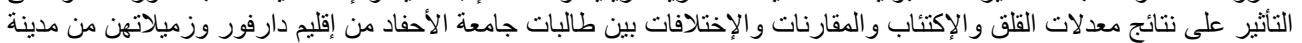

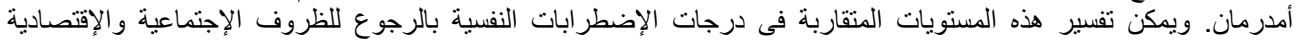

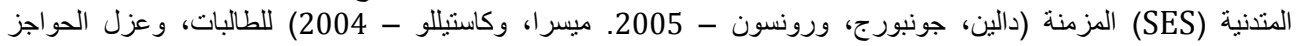

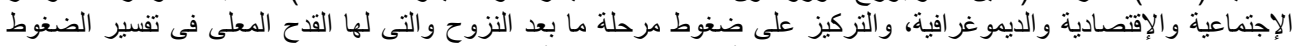

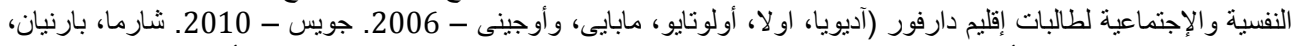
وسبيلبيرجر - 1983) وأعراض "القلق التوقعي" (Anticipatory Anxiety) لطالبات مدينة أمدرمان (ثابت، عابد، دارئ،

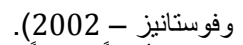

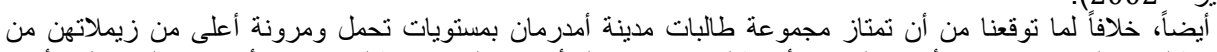

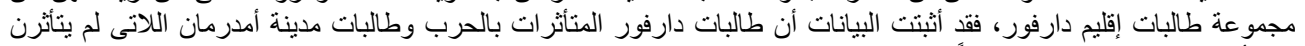

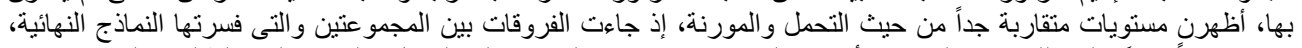

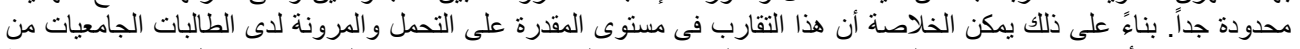

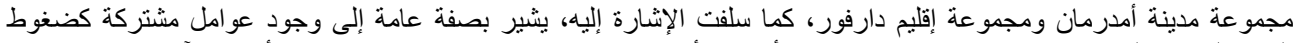

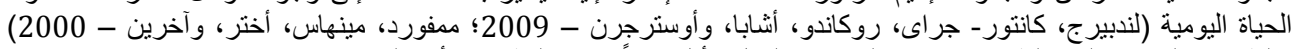

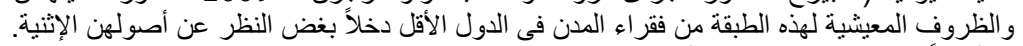

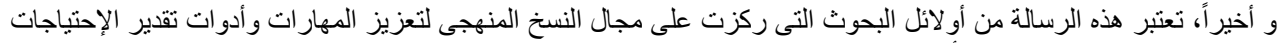

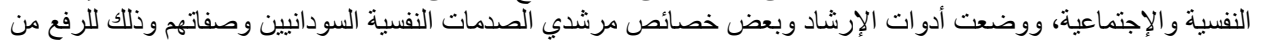

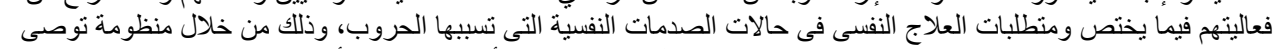

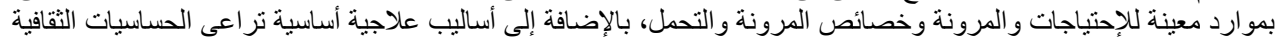

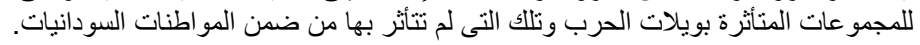


Appendices 


\section{Appendix 1}

\section{INTERVIEW PROTOCOL (1)}

General information guidelines:

Site of data collection: Ahfad University for Women (AUW) School of Organizational; Management, School of Psychology, School of Health Sciences, School of REED, School of Medicine, School of Pharmacy.

Date(s) of data collection: 17-30 Dec 2008

Sample: DARFURI AUW UNDRGRADUATE STUDENTS (17-25 years old)

Data collector/Interviewer: Alia Badri (Principal Researcher)

Prompt Questions for Semi-structured interviews

THE EFFECTS OF WAR EXPERIENCES/ TRAUMATIC WAR EXPOSURE:

(I am not seeking any psychologically related responses, but responses such as "my family is uprooted, we are separate, loss of possessions/people")

- Could you tell me what you know about the war in Darfur? Prompts- I understand it has been some time since the war began, but do you recall when it began; where it is happening (village/town name)

- Could you tell me how the war in Darfur province directly affected you and your family? Prompts- how did it start; when did it start;

- Could you tell me about your experiences in the Darfur conflict? Prompts- did you live close to any fighting; did you hear people talk about their fighting; did you see/hear any fighting

- Do you see yourself as an uprooted/ internally displaced person? Are your parents/family?

-During the war were you in a situation where you were: SELF

Severely injured (where/how); Sexually assaulted; Tortured or assaulted (non-sexually); Been close to shooting bullets; Been close to bomb or grenade explosions; Threatened of being killed or severely injured; Captured (how); Detained (how/where); Without food or water (how long);

-During the war were you a witness to: LOVED ONE/OTHER

A loved one being killed; Dead body (ies) of loved one(s); Dead body(ies); Torture or physical assault; Someone being sexually assaulted; A severely injured person; Being kidnapped/abducted; Being detained/imprisoned

-During the war were you a witness to Losses: NUCLEAR/EXTENDED

Was your Father/ Mother/ Brother/Sister/Uncle/ Aunt/Grandparents/Cousins killed? PSYCHOLOGICAL RESPONSES

-How did/does this war affect you emotionally? Prompts- how do you feel: sad, lonely, grieved, apprehensive, anxious, guilty

- How are you feeling now that you are separated (uprooted) from your family and not knowing their status?

Post-displacement Experiences

- Could you tell me how you got to Khartoum state/Omdurman city? What were the events that led you to coming to Omdurman/Ahfad? How did you get here? Prompts- means of transportation; choice; forced

-What would you say was-is the worst event(s) that you have experienced being in Khartoum state/Omdurman city? Prompts- how difficult is life for you nowadays; 
-How would you describe your feelings nowadays?

-What are your real/actual life changes? What are your differences in life style? Prompts- what do you have there that you don't have here

-How would you rate these life changes that you are experiencing? Prompts- describe actual events that are different

-Are you stressed? What are your stressors? Could you give me an example of your worst experience in Omdurman? Prompt- Actual event that occurred; day or night; on campus or off; with whom (worse experiences living in Omdurman)

- How would they rate these stressful life experiences? Do you feel there is a cultural incongruence's between you and people in Omdurman? Prompt-Person environment fit

- How did this experience make you feel? Prompt- psychological description

-What are your academic barriers/ how would you rate your academic achievements?

\section{Coping Strategies}

-You have experienced events in your life that many people might describe as difficult or stressful. How did (do you) deal with or cope with those events? Prompts-describe your behaviors; what do you do typically, who do you turn to, how do you feel, what do you need the most

- How do you deal with events that are happening to you now in Omdurman? Prompts- describe your behaviors; what do you do typically, who do you turn to, how do you feel, what do you need the most nowadays

-What sort of social relationships do you have here in Omdurman? Prompt- role models; time spent; amount shared; ethnically similar

- How do they make you make you feel? Prompt- psychological description

-Many people might describe someone who dealt with or coped well with these events as strong or resilient. What helped you in being (or becoming) resilient or strong?

(The participant might mention individual, familial or community protective factors. I will acknowledge all the responses and will focus on whichever protective factor(s) the participant mention the most.)

-In what ways did your coping style or strategy help you in dealing with your stressors? How well has it worked for you? Could you give me an example? Then (Darfur)? Now (Omdurman)?

(I am looking for a descriptive process)

-Is there anything that you think is important for me to know about your coping strategy/ factors/ determinants that I have not asked? Or is there anything else you would like to add? 


\section{Appendix 2}

\section{Demographic Questionnaire}

Question

Name

Age

Semester

School (Department)

Place of origin

Which part of Darfur are you from? (N/S/W)

Date of arrival in Khartoum/Omdurman

How did you get here

Where do you live now

With whom do you live now

Do you have relatives here in Khartoum

Where are these relatives living

Where are your parents

Is your father alive

Where is he living

What does he do

Is your mother alive

Where is she living

What does she do

Who pays your tuition fees

Tribe name

Village/town name

Does your family own property in your place of origin

Where

Has your family lost their property

When

How

Has your family been uprooted or displaced

Why have they been displaced

When have they left their place of origin

Have you been uprooted or displaced
Response 
How many sisters do you have

How many brothers do you have

What is your birth order $\left(1^{\text {st }} / 2^{\text {nd }} / 3^{\text {rd }} / 4^{\text {th }}\right)$

Have you been in Khartoum before

When were you in Khartoum

Why did you come to Khartoum

How long did you stay in Khartoum

Why did you leave Khartoum

Where did you go to

Why did you choose Ahfad University for your studies

Have you searched for any other university

What will you do when you graduate 


\section{Appendix 3}

\section{HARVARD TRAUMA QUESTIONNAIRE}

Date:

Subject Number:

Age:

Instructions: We would like to ask you about your past history and present symptoms. You may find some questions upsetting. If so, please feel free not to answer. The answers to the questions will be kept confidential.

Part 1: Trauma Events. Please indicate whether you have experienced, witnessed, or heard of any of the following events.
$\mathrm{E}=$ Experienced
$\mathrm{W}=\mathrm{Witnessed}$
$\mathrm{H}=$ Heard about it
$\mathrm{N}=\mathrm{No}$

\begin{tabular}{|c|c|c|c|c|c|}
\hline & Event & $\mathrm{E}$ & W & $\mathrm{H}$ & $\mathrm{N}$ \\
\hline 1 & Lack of shelter/ no where to live & & & & \\
\hline 2 & Movement in search of shelter & & & & \\
\hline 3 & Forced to hide & & & & \\
\hline 4 & Constant insecurity & & & & \\
\hline 5 & Lack of food or water & & & & \\
\hline 6 & Living in displaced camps & & & & \\
\hline 7 & Exposed to unhygenic conditions & & & & \\
\hline 8 & Poor health without access to medical care & & & & \\
\hline 9 & Loss of health & & & & \\
\hline 10 & Increase in physical ailments & & & & \\
\hline 11 & Confiscation or destruction of personal property & & & & \\
\hline 12 & Burning of home & & & & \\
\hline 13 & Not having right to a home & & & & \\
\hline 14 & Owning nothing & & & & \\
\hline 15 & Complete change of life & & & & \\
\hline 16 & Combat situation (e.g. shelling and grenade attacks) & & & & \\
\hline 17 & Arial bombings (planes and helicopters) & & & & \\
\hline 18 & Looting of nearby villages & & & & \\
\hline 19 & Forced evacuation under dangerous conditions & & & & \\
\hline 20 & Fleeing/ running away & & & & \\
\hline 21 & Beating to the body & & & & \\
\hline 22 & Rape & & & & \\
\hline
\end{tabular}




\begin{tabular}{|c|c|c|c|c|c|}
\hline & Event & $\mathrm{E}$ & W & $\mathrm{H}$ & $\mathrm{N}$ \\
\hline 23 & Other types of sexual abuse or sexual humiliation & & & & \\
\hline 24 & Innocent victims being killed & & & & \\
\hline 25 & Hearing of atrocities & & & & \\
\hline 26 & Seeing dead bodies & & & & \\
\hline 27 & Knifing/ axing/ slaughtering & & & & \\
\hline 28 & Harshness and cruel treatment ( physical or mental suffering) & & & & \\
\hline 29 & Serious physical injury from combat situation or landmine & & & & \\
\hline 30 & Imprisonment & & & & \\
\hline 31 & Kidnapped & & & & \\
\hline 32 & Suddenly gone missing & & & & \\
\hline 33 & Parentless children/ teenagers & & & & \\
\hline 34 & Disappearance or kidnapping of family member or friend & & & & \\
\hline 35 & Forced separation from family members & & & & \\
\hline 36 & Distance from family & & & & \\
\hline 37 & Enforced isolation from others & & & & \\
\hline 38 & Extortion \& robbery & & & & \\
\hline 39 & Finding dead bodies and burying them & & & & \\
\hline 40 & No feedomn of movement because fear for life & & & & \\
\hline 41 & $\begin{array}{l}\text { Forced to physically harm someone ( e.g. family member, friend, } \\
\text { stranger) }\end{array}$ & & & & \\
\hline 42 & Forced to destroy someone else's property or possessions & & & & \\
\hline 43 & $\begin{array}{l}\text { Serious physical injury of family member or friend due to combat } \\
\text { situation orlandmine }\end{array}$ & & & & \\
\hline 44 & Murder, or death due to violence, of family member or friend & & & & \\
\hline 45 & Witness beatings to head or body & & & & \\
\hline 46 & Prevented from burying someone & & & & \\
\hline 47 & Exposed to threats, humiliation, or discrimination & & & & \\
\hline 48 & Exposed to heat, sun, strong light & & & & \\
\hline 49 & $\begin{array}{l}\text { Someone was forced to betray you and place you at risk of death or } \\
\text { injury }\end{array}$ & & & & \\
\hline
\end{tabular}


Part II: Personal Description

Please indicate what you consider the most hurtful or terrifying event(s) related to the current conflict happening within the Darfur region that you have experienced. Please specify where and when these events occurred.

Under your current living situation (i.e. parental home, extended family home, student housing) what is the worst event that has happened to you, in Khartoum. Please specify where and when these events occurred. 


\section{Part IV: Trauma Symptoms}

The following are symptoms that people sometimes have after experiencing hurtful or terrifying events in their lives. Please read each one carefully and decide how much the symptoms bothered you in the past 6-12 months.

\begin{tabular}{|c|c|c|c|c|c|}
\hline & & Not at all & A little & Quite a bit & Extremely \\
\hline 1. & $\begin{array}{l}\text { Recurrent thoughts or memories of the most hurtful } \\
\text { or terrifying events }\end{array}$ & 1 & 2 & 3 & 4 \\
\hline 2. & Feeling as though the event is happening again & & & & \\
\hline 3. & Recurrent nightmares & & & & \\
\hline 4. & Feeling detached or withdrawn from people & & & & \\
\hline 5. & Unable to feel emotions (numbness) & & & & \\
\hline 6. & Feeling jumpy easily startled & & & & \\
\hline 7. & Difficulty concentrating & & & & \\
\hline 8. & Trouble sleeping & & & & \\
\hline 9. & Feeling on guard & & & & \\
\hline 10. & Feeling irritable or having outbursts of anger & & & & \\
\hline 11. & $\begin{array}{l}\text { Avoiding activities that remind you of the traumatic } \\
\text { or hurtful event }\end{array}$ & & & & \\
\hline 12. & $\begin{array}{l}\text { Inability to remember parts of the most traumatic or } \\
\text { hurtful events }\end{array}$ & & & & \\
\hline 13. & Less interest in daily activities & & & & \\
\hline 14. & Feeling as if you don't have a future & & & & \\
\hline 15. & $\begin{array}{l}\text { Avoiding thoughts or feelings associated with the } \\
\text { traumatic or hurtful experience }\end{array}$ & & & & \\
\hline 16. & $\begin{array}{l}\text { Sudden emotional or physical reaction when re- } \\
\text { minded of the most hurtful or traumatic events }\end{array}$ & & & & \\
\hline 17. & Feeling that you have less skills than you had before & & & & \\
\hline 18. & Having difficulty dealing with new situations & & & & \\
\hline 19. & Feeling exhausted & & & & \\
\hline 20. & Bodily pain & & & & \\
\hline 21. & Troubled by physical problem(s) & & & & \\
\hline 22. & Poor memory & & & & \\
\hline 23. & $\begin{array}{l}\text { Finding out or being told by other people that you } \\
\text { have done something that you cannot remember }\end{array}$ & & & & \\
\hline 24. & Difficulty paying attention & & & & \\
\hline 25. & Feeling miserable & & & & \\
\hline
\end{tabular}




\begin{tabular}{|c|c|c|c|c|c|}
\hline & & Not at all & A little & Quite a bit & Extremely \\
\hline 26. & Feeling unable to make daily plans & & & & \\
\hline 27. & Blaming yourself for things that have happened & & & & \\
\hline 28. & Feeling guilty for having survived. & & & & \\
\hline 29. & Hopelessness & & & & \\
\hline 30. & $\begin{array}{l}\text { Feeling ashamed of the hurtful or traumatic events } \\
\text { that have happened to you }\end{array}$ & & & & \\
\hline 31. & $\begin{array}{l}\text { Feeling that people do not understand what hap- } \\
\text { pened to you. }\end{array}$ & & & & \\
\hline 32. & Feeling others are hostile to you & & & & \\
\hline 33. & Feeling as if you are going crazy & & & & \\
\hline 34. & Feeling isolated because of loss of social role & & & & \\
\hline 35. & $\begin{array}{l}\text { Feeling isolated because of loss of relationships with } \\
\text { family, neighbors and friends }\end{array}$ & & & & \\
\hline 36. & Feeling afraid to fall asleep out of intense fear & & & & \\
\hline 37. & Feeling overly dependent on others & & & & \\
\hline 38. & $\begin{array}{l}\text { Feeling discriminated against because of loss of } \\
\text { home and social status. }\end{array}$ & & & & \\
\hline 39. & $\begin{array}{l}\text { Only feeling normal among other people who also } \\
\text { suffered the same sort of trauma }\end{array}$ & & & & \\
\hline 40. & Feeling that you have no one to rely on. & & & & \\
\hline 41. & Feeling someone you trusted betrayed you. & & & & \\
\hline 42. & Feeling humiliated by your experiences & & & & \\
\hline 43. & Feeling no trust in others (distrust) & & & & \\
\hline 44. & Feeling powerless to help yourself or others & & & & \\
\hline 45. & $\begin{array}{l}\text { Spending time thinking about why these events } \\
\text { happened to you. }\end{array}$ & & & & \\
\hline 46 & $\begin{array}{l}\text { Feeling that you are the only one who suffered these } \\
\text { events. }\end{array}$ & & & & \\
\hline
\end{tabular}


توجيهات: الأسئلة التالية خاصة بتجاربك السابقة الأكثر ايلاما وقساوة وكيف تشعرين بنتيجتها الآن. يمكنك أن تجدي بعض من الاسئلة تزعجك ولذا سوف تظل اجابنك سرية. الرجاء الاجابة على جميع الاسئلة ولا تتركي أبي فراغ، وشكرا لحسن تعاونك معنا.

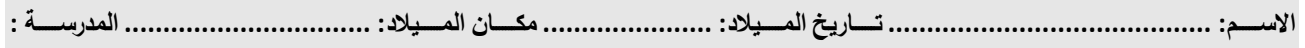

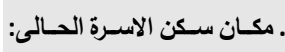

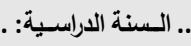

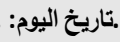

القسم الأول: أحداث الصدمة: الص

الرجاء توضيح الأحداث التالية إذا ما كتبتي قد حربتيها أو شاهدتيها أو سمعت عنها

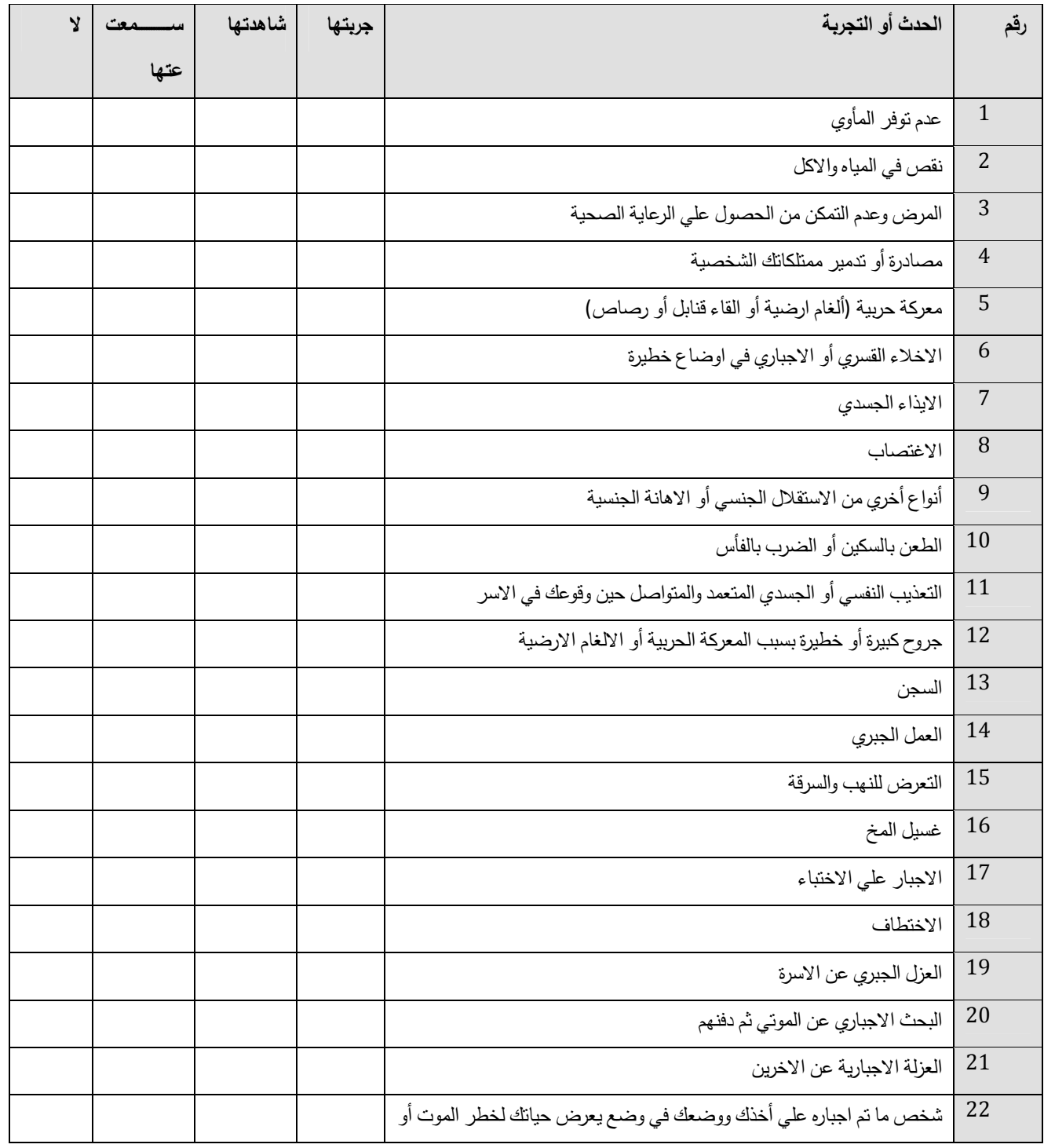




\begin{tabular}{|c|c|c|c|c|c|}
\hline ע & عتها & شاهنتها & 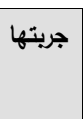 & الحدث أو التجرية & رقم \\
\hline & & & & الاصابة & \\
\hline & & & & منعت من دفن أحد الموتي & 23 \\
\hline & & & & أجبرت علي نبش أو تحطيم قبور الموتي & 24 \\
\hline & & & & أجبرت علي ايذاء أفراد اسرتك أو أصدقائك & 25 \\
\hline & & & & أجبرت علي ايذاء شخص من خارج اسرتك & 26 \\
\hline & & & & أجبرت علي تحطيم ممتلكات وحيازات الاخرين & 27 \\
\hline & & & & أجبرت علي خيانة اسرتك ووضعهم في موضع يعرضهم للموت أو الايذاء & 28 \\
\hline & & & & أجبرت علي خيانة شخص من غير اسرتك ووضعه في موضع يعرضه للموت او & 29 \\
\hline & & & & مقتل او موت أحد من أفراد اسرتك أو اصدقائك بسبب العنف & 30 \\
\hline & & & & اختفاء أو اختطاف أحد من أفراد اسرتك أو اصدقائك & 32 \\
\hline & & & & الارضية بـابة بليغة أو كبيرة لأحـد أفراد عائلتك أو أصدقائك بسبب الحرب أو الالغـام & 32 \\
\hline & & & & شاهنتي ضرب في الرأس أو الجسم لأحد ما & 33 \\
\hline & & & & شاهنتي تعذيب & 34 \\
\hline & & & & التعرض للتهديد والاهانة & 35 \\
\hline & & & & التعرض الي الثمس والاهانة الثديدة & 36 \\
\hline & & & & التعرض الي البرد الثنديد والامطار الغزيرة & 37 \\
\hline & & & & الاجبار علي العمل المضني والثشاق & 38 \\
\hline & & & & التعرض لأوضاع غير صحية تسبب للك الاصابة بالامراض & 39 \\
\hline & & & & الاجبار علي كتابة او توقيع اقرار لم تقومين به & 40 \\
\hline
\end{tabular}

القسم الثاني: التجارب الثخصية:

1/ أرجو سرد أرعب المواقف التي مرت بك في المعركة التي تدور في منطقة دارفور (ان وجد)، الرجاء نوضيح الزمان والمكان الذي حدثت فيه تلك

المواقف؟

2/ في حياتك الحالية ما هي أسوأ المواقف صعوبة وأذية التي تعرضتي لها أومرت بك أو حدثث للك في الخرطوم (ان وجد)؟ حددي المكان والزمان الذي

حدثت فيه؟ 
القسم الرابع: أعراض الصدمة النفسية:

في ما يلي أعراض قد تحدث بعد مرور الثخص بتجربة (صدمة) نفسية، مؤلمة ومخيفة في حياتهم، الرجاء قراءة كل سؤال بعناية ثُ توضيح كيف أثر فيك سلباً كل عرض من أعراض الصدمة خلال الفترة الماضية (6 - 12 شهر ).

\begin{tabular}{|c|c|c|c|c|c|}
\hline اطلاقاً & 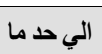 & كثير & كثير جدا & الاعراض & رقم \\
\hline & & & & التفكير المستمر في التجارب الاكثر ايلاما وقساوة مرة بعد مرة & 1 \\
\hline & & & & الإحساس بتكرار التجارب الاكثر ايلاما وقساوة مرة بعد مرة & 2 \\
\hline & & & & استمرارية توارد الكوابيس & 3 \\
\hline & & & & الشعور بالعزلة والانفصال عن الآخرين & 4 \\
\hline & & & & عدم القدرة على الاحساس بالعواطف & 5 \\
\hline & & & & الاحساس بالخلعة (الذعر والهلع) وبسهولة من أي صوت & 6 \\
\hline & & & & عدم القدرة علي التركيز في الأشياء & 7 \\
\hline & & & & اضطرابات في النوم & 8 \\
\hline & & & & الإحساس بالتحفظ & 9 \\
\hline & & & & الشعور بالتوتر والنرفذة أو أنك على حافة الانفجار من الغضب & 10 \\
\hline & & & & تجنب النشاطات التي تذكرك بالأحداث الاكثر ايلاما وقساوة & 11 \\
\hline & & & & العجز عن تذكر جزءمن الأحداث الاكثر ايلاماوقساوة & 12 \\
\hline & & & & أقل رغبةً في القيام بالنشاطات الحياتية اليومية & 13 \\
\hline & & & & الشعور بأن لا مستقبل لديك & 14 \\
\hline & & & & تتحاشي الأفكار والمشاعر المرتبطة بالأحداث الاكثر ايلاما وقساوة & 15 \\
\hline & & & & تصابين برد فعل عاطفي وجسماني فجائي حينما تتذكري الأحداث & 16 \\
\hline & & & & الثعور بأنك أصبحت أقل مهارة عن السابق أو ما كنت عليها & 17 \\
\hline & & & & تجدين صعوبة في التعامل مع المواقف الجديدة & 18 \\
\hline
\end{tabular}




\begin{tabular}{|c|c|c|c|c|c|}
\hline اطلاقاً & 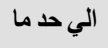 & كثير & كثير جدا & | الاعراض & رقم \\
\hline & & & & الإحساس بالارهاق & 19 \\
\hline & & & & تحسين بالألام جسدياً & 20 \\
\hline & & & & 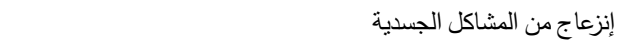 & 21 \\
\hline & & & & ضعف في الذاكرة & 22 \\
\hline & & & & تكتشفين أو يخبرك الآخرين بأنك قد قمت بعمل شيء ولكنك لا تتذكرينه & 23 \\
\hline & & & & تجدين صعوبة في الانتباه & 24 \\
\hline & & & & تتشعرين وكأنك قد انقسمت إلى شخصين أحدهما يراقب ما يفعله الآخر & 25 \\
\hline & & & & الاحساس بعدم القدرة علي وضع خططك اليومية & 26 \\
\hline & & & & تأنبين نفسك علي أثنياء حدثت & 27 \\
\hline & & & & الثعور بالذنب علي البقاء حية & 28 \\
\hline & & & & 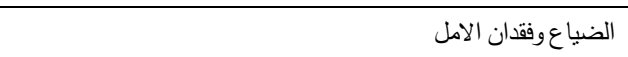 & 29 \\
\hline & & & & الاحساس بالعار من الأحداث المؤلمة التي مررت بها & 30 \\
\hline & & & & الاحساس بعدم فهم الاخرين للأحداث التي ممرت بها & 31 \\
\hline & & & & 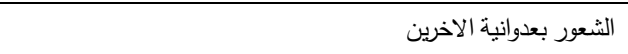 & 32 \\
\hline & & & & الثعور بفقدان العقل (الجنون) & 33 \\
\hline & & & & الشعور بالعزلة بسبب فقدان الدور الاجتماعي & 34 \\
\hline & & & & الشعور بالعزلـة بسبب فقدان العلاقات الاجتماعيـة (علاقات الاسـرة، & 35 \\
\hline & & & & الشعور بالخوف في الاستغراق في النوم بسبب الذعر & 36 \\
\hline & & & & الافراط في الاعتماد علي الاخرين & 37 \\
\hline & & & & الاحساس بالتمييز والاضطهاد لفقدك الموطن والوضع الاجتماعي & 38 \\
\hline & & & & لالاحساس بالالفة فقط بين اللاتي تعرضن لنفس الظروف التي تعرضني & 39 \\
\hline & & & & الثعور بأن ليس لديك أحدا تعتمدين عليه & 40 \\
\hline & & & & الاحساس بأنك كنتي تعتمدين علي شخص وتثقين به ثم خذلك & 41 \\
\hline
\end{tabular}




\begin{tabular}{|c|c|c|c|c|c|}
\hline اطلاقاً & 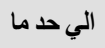 & كثير & كثير جدا & الاعراض & رقم \\
\hline & & & & الشعور بالاذلال من تعرضك لتلك الأحداث المؤلمة التي مررت بها & 42 \\
\hline & & & & الثعور بعدم الوثوق بالاخرين & 43 \\
\hline & & & & الشعور بعدم القدرة علي مساعدة الاخرين & 44 \\
\hline & & & & تمضية الوقت الكثير في مسائلة نفسك لماذا حدثت لي هذه الأثشياء & 45 \\
\hline & & & & الثعور بأنني الثخص الوحيد الذي عاني من هذه التجربة المريرة & 46 \\
\hline
\end{tabular}




\section{Appendix 4}

\section{Hopkins checklist-25}

Date:__________ Subject Number:____ Age:

Listed below are some symptoms of strain that people sometimes have. Please read each one carefully and circle the answer that best reflects how much that symptom has bothered you during the past 6-12 months.

\begin{tabular}{|c|c|c|c|}
\hline \multirow{2}{*}{ Q. } & 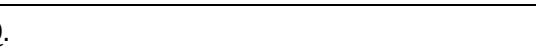 & \multirow[t]{2}{*}{ Not at all } & \multirow[t]{2}{*}{ Quite a bit } \\
\hline & 1 Suddenly scared for no reason & & \\
\hline 2 & \multicolumn{3}{|l|}{ Feeling fearful } \\
\hline 3 & \multicolumn{3}{|l|}{ Faintness, dizziness, or weakness } \\
\hline 4 & \multicolumn{3}{|l|}{ Nervousness or shakiness inside } \\
\hline 5 & \multicolumn{3}{|l|}{ Heart pounding or racing } \\
\hline 6 & \multicolumn{3}{|l|}{ Trembling } \\
\hline 7 & \multicolumn{3}{|l|}{ Feeling tense or keyed up } \\
\hline 8 & \multicolumn{3}{|l|}{ Headaches } \\
\hline 9 & \multicolumn{3}{|l|}{ Spells of terror or panic } \\
\hline 10 & \multicolumn{3}{|l|}{ Feeling restless, can't sit down } \\
\hline 11 & \multicolumn{3}{|l|}{ Feeling low in energy - slowed down } \\
\hline 12 & \multicolumn{3}{|l|}{ Blaming yourself for things } \\
\hline 13 & \multicolumn{3}{|l|}{ Crying easily } \\
\hline 14 & \multicolumn{3}{|l|}{ Loss of sexual interest or pleasure } \\
\hline 15 & \multicolumn{3}{|l|}{ Poor appetite } \\
\hline 16 & \multicolumn{3}{|l|}{ Difficulty falling asleep or staying asleep } \\
\hline 17 & \multicolumn{3}{|l|}{ Feeling hopeless about the future } \\
\hline 18 & \multicolumn{3}{|l|}{ Feeling blue } \\
\hline 19 & \multicolumn{3}{|l|}{ Feeling lonely } \\
\hline 20 & \multicolumn{3}{|l|}{ Feeling trapped or caught } \\
\hline 21 & \multicolumn{3}{|l|}{ Worrying too much about things } \\
\hline 22 & \multicolumn{3}{|l|}{ Feeling no interest in things } \\
\hline 23 & \multicolumn{3}{|l|}{ Thoughts of ending your life } \\
\hline 24 & \multicolumn{3}{|l|}{ Feeling everything is an effort } \\
\hline 25 & \multicolumn{3}{|l|}{ Feelings of worthlessness } \\
\hline
\end{tabular}


توجيهات:

في القائمة أدناه بعض الاعراض والمشاكل التي يشعر بها الناس في بعض الاحيان نتيجة لقساوة التجارب المؤلمة والمزعجة. الرجاء قراءة كل منها جيدا ووضع علامة صح ( V ) أمام الاجابة التي تعكس مدي تأثرك بالاعراض أو المشاكل المذكورة خلال الفترة الماضية (6 الي 12 شهر). نرجو أن لا تتركي أي فراغات والاجابة علي جميع الاسئلة. وشكرا علي حسن تعاونك معنا.

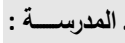

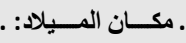
... تـــاريخ المـــلاد: ... الاســـــ . . ـ مكــان سـكن الاسـرة الحـالىى مكسان الـسكن الحسالى: .

. السسنة الدراسسية: . . . .

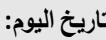

\begin{tabular}{|c|c|c|c|c|c|}
\hline كثير جدا & كثير & الي حد ما الي & اطلاقةاً & الأعراض & رقم \\
\hline & & & & أشعر بالخوف فجأةومن غير سبب & 1 \\
\hline & & & & الشعور بالخوف & 2 \\
\hline & & & & الثعور بالغتيان أو إغماء، أو دوار ، أو ضعف عام & 3 \\
\hline & & & & الشعور بالقلق ورجفة داخلية & 4 \\
\hline & & & & الشعور بتسارع ضربات القلب & 5 \\
\hline & & & & الشعور بالارتعاش & 6 \\
\hline & & & & التوتز والعصبية & 7 \\
\hline & & & & الصداع & 8 \\
\hline & & & & الشعور بنوبة رعب أو ذعر & 9 \\
\hline & & & & الثعور بعدم الارتياح وعدم الاستقرار & 10 \\
\hline & & & & الشعور بهبوط في الطاقة والبطء & 11 \\
\hline & & & & القيام بلوم نفسك & 12 \\
\hline & & & & البكاء لأبسط الاسباب & 13 \\
\hline & & & & الثعور بعدم الرغبة الجنسية والمتعة & 14 \\
\hline & & & & ضعف في الشهية & 15 \\
\hline
\end{tabular}




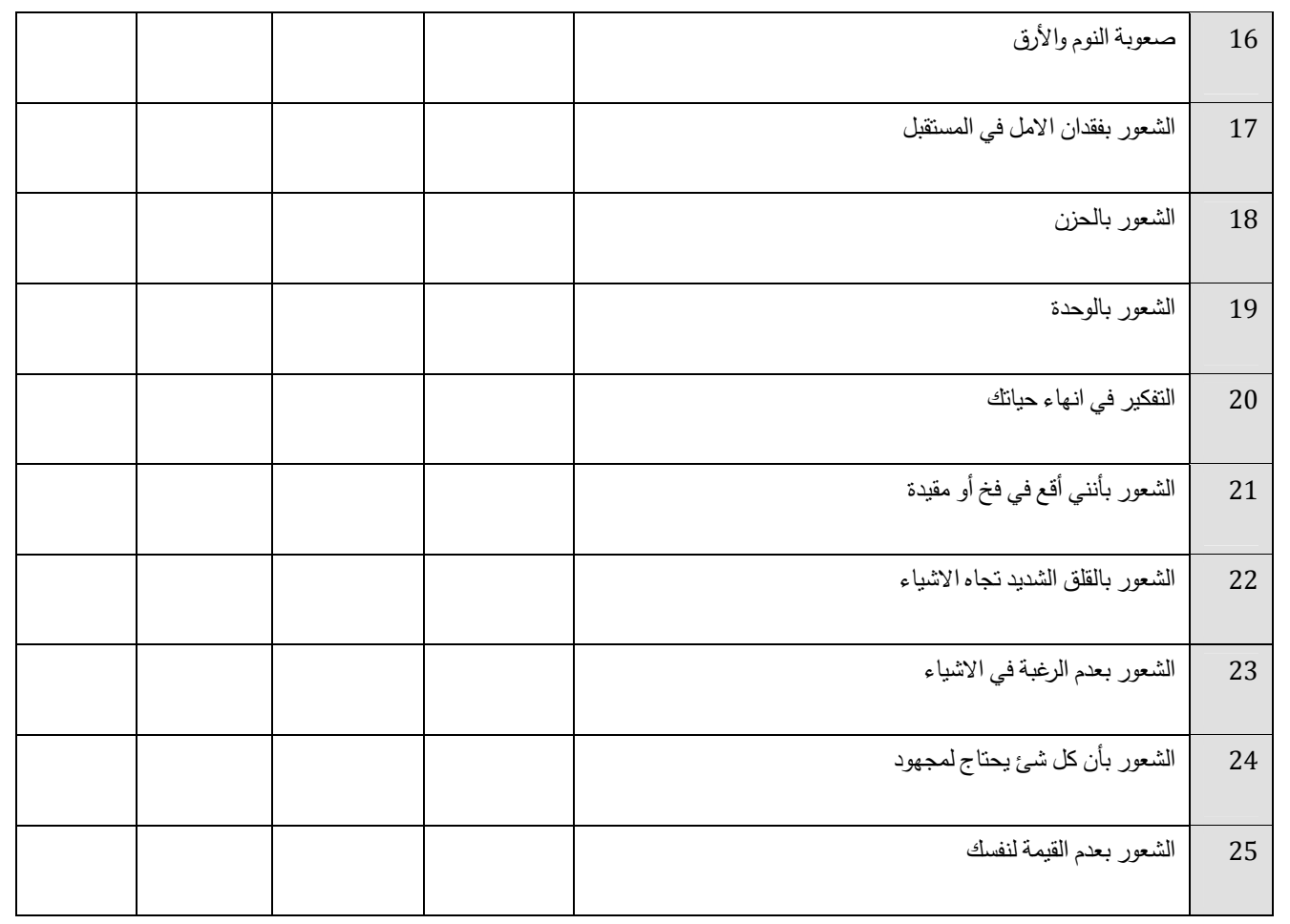




\section{Appendix 5}

\section{Resilience scale}

Date:

Subject Number:

Age:

Please read the following statements. To the right of each you will find seven numbers, ranging from "1" (Strongly Disagree) on the left to "7" (Strongly Agree) on the right. Circle the number which best indicates your feelings about that statement. For example, if you strongly disagree with a statement, circle "1". If you are neutral, circle "4", and if you strongly agree, circle "7", etc.

\begin{tabular}{|c|c|c|c|c|c|c|c|}
\hline \multirow[b]{2}{*}{ 1. When I make plans, I follow through with them. } & \multicolumn{4}{|c|}{$\begin{array}{l}\text { Strongly } \\
\text { Disagree }\end{array}$} & \multicolumn{3}{|c|}{$\begin{array}{r}\text { Strongly } \\
\text { Agree }\end{array}$} \\
\hline & 1 & 2 & 3 & 4 & 5 & 6 & 7 \\
\hline 2. I usually manage one way or another. & 1 & 2 & 3 & 4 & 5 & 6 & 7 \\
\hline 3. I am able to depend on myself more than anyone else. & 1 & 2 & 3 & 4 & 5 & 6 & 7 \\
\hline 4. Keeping interested in things is important to me. & 1 & 2 & 3 & 4 & 5 & 6 & 7 \\
\hline 5. I can be on my own if I have to. & 1 & 2 & 3 & 4 & 5 & 6 & 7 \\
\hline 6. I feel proud that I have accomplished things in life. & 1 & 2 & 3 & 4 & 5 & 6 & 7 \\
\hline 7. I usually take things in stride. & 1 & 2 & 3 & 4 & 5 & 6 & 7 \\
\hline 8. I am friends with myself. & 1 & 2 & 3 & 4 & 5 & 6 & 7 \\
\hline 9. I feel that I can handle many things at a time. & 1 & 2 & 3 & 4 & 5 & 6 & 7 \\
\hline 10. I am determined. & 1 & 2 & 3 & 4 & 5 & 6 & 7 \\
\hline 11. I seldom wonder what the point of it all is. & 1 & 2 & 3 & 4 & 5 & 6 & 7 \\
\hline 12. I take things one day at a time. & 1 & 2 & 3 & 4 & 5 & 6 & 7 \\
\hline $\begin{array}{l}\text { 13. I can get through difficult times because I've experienced difficulty } \\
\text { before. }\end{array}$ & 1 & 2 & 3 & 4 & 5 & 6 & 7 \\
\hline 14. I have self-discipline. & 1 & 2 & 3 & 4 & 5 & 6 & 7 \\
\hline 15. I keep interested in things. & 1 & 2 & 3 & 4 & 5 & 6 & 7 \\
\hline 16. I can usually find something to laugh about. & 1 & 2 & 3 & 4 & 5 & 6 & 7 \\
\hline 17. My belief in myself gets me through hard times. & 1 & 2 & 3 & 4 & 5 & 6 & 7 \\
\hline 18. In an emergency, I'm someone people can generally rely on. & 1 & 2 & 3 & 4 & 5 & 6 & 7 \\
\hline
\end{tabular}




\begin{tabular}{|l|l|l|l|l|l|l|l|}
\hline 19. I can usually look at a situation in a number of ways. & 1 & 2 & 3 & 4 & 5 & 6 & 7 \\
\hline 20. Sometimes I make myself do things whether I want to or not. & 1 & 2 & 3 & 4 & 5 & 6 & 7 \\
\hline 21. My life has meaning. & 1 & 2 & 3 & 4 & 5 & 6 & 7 \\
\hline 22. I do not dwell on things that I can't do anything about. & 1 & 2 & 3 & 4 & 5 & 6 & 7 \\
\hline 23. When I'm in a difficult situation, I can usually find my way out of it. & 1 & 2 & 3 & 4 & 5 & 6 & 7 \\
\hline 24. I have enough energy to do what I have to do. & 1 & 2 & 3 & 4 & 5 & 6 & 7 \\
\hline 25. It's okay if there are people who don't like me. & 1 & 2 & 3 & 4 & 5 & 6 & 7 \\
\hline 26. I am resilient. & 1 & 2 & 3 & 4 & 5 & 6 & 7 \\
\hline
\end{tabular}

(C) 1987 Gail M. Wagnild \& Heather M. Young. Used by permission. All rights reserved. "The Resilience Scale" is an international trademark of Gail M. Wagnild \& Heather M. Young. 


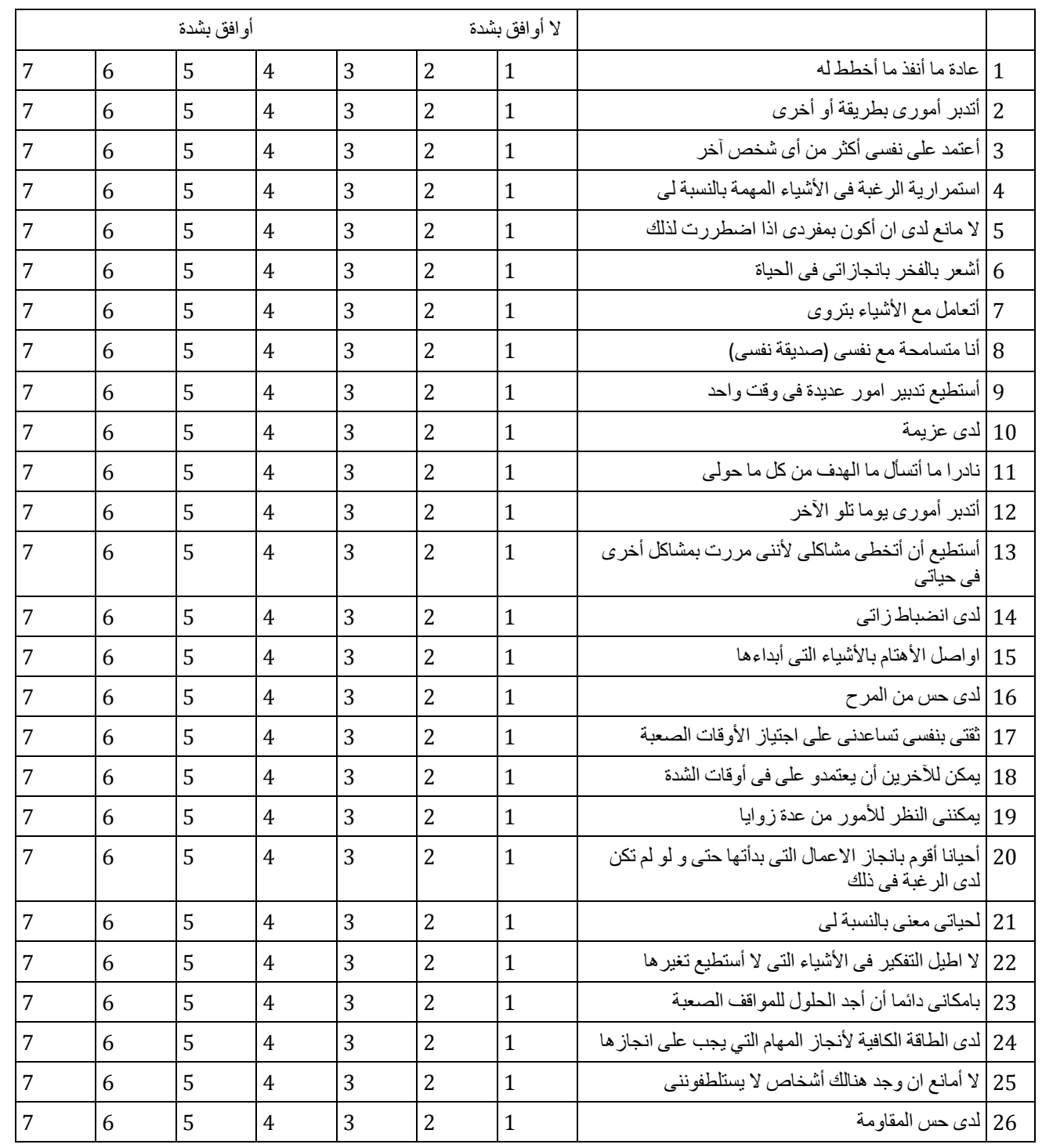

توجيهات: الرجاء قراعة الجمل ادناه بعناية. في الجهة اليمنى من الجمل ستجدين سبعة أرقام, من الرقم 1- 7 و التى تمثل الحالات (عدم الموافقة.

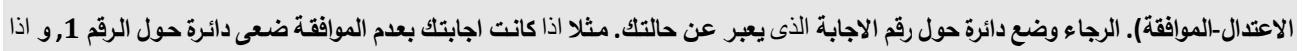
كانت بالاعتدال ضعى دائرة حول الرقم4, و فى حالة الاجابة بالموافقة ضعى دائرة حول الرقم 7. : المدرسة مكان الميلاد: تاريخ الميلاد: الاسم: مكان سكن الاسرة الحالى:

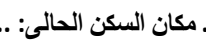
. 


\section{Bibliography}

Abela, J. R. Z., \& Hankin, B. L. (2009). Cognitive vulnerability to depression in adolescents: A developmental psychopathology perspective. In S. Nolen-Hoeksema \& L. Hilt (Eds.), Handbook of adolescent depression (pp.:335-376). Hillsdale, NJ: Lawrence Erlbaum.

Abu-Saba, M. B. (1999). War-related trauma andstress characteristics of American university of Beirut students. Journal of Traumatic Stress, 12, 201-207.

Adewuya, A. O., Ola, B. A., Olutayo, O. A., Mapayi, B. M., \& Oginni, O. O. (2006). Depression amongst Nigerian university students. Prevalence and socio-demographic correlates. Social Psychiatry and Psychiatric Epidemiology, 41.

Adjukovic, M. (1998). Displaced adolescents in Croatia: Sources of stress and posttraumatic stress reaction. Adolescence, 33, 209-217.

Aldwin, C., \& Greenberger, E. (1987). Cultural differences in the predictors of depression. American Journal of Community Psychology, 15, 789-813.

American Psychiatric Association. (1994). Diagnostic and Statistical Manual of Mental Health (Vol. 4th ed.). Washington DC: American Psychiatric Press.

American Psychological Association. (2000). Diagnostic and statistical manual of mental disorders; DSM IV $T R$. Washington.

Armenian, H. K., Morikawa, M., \& Melkonian, A. K. (2002). Risk factors for depression in the survivors of the 1988 earthquake in Armenia. Journal in Urban Health, 79, 373-382.

Arotz-Badenan, R. D., \& Olvin, P. E. (1986). A Rural-Urban Comparison. Family Relations, 35, 281-288.

Assimeng, M. (1981). Social structure of Ghana: A study in persistence and change. Ghana Tema: Ghana Publishing Corporation.

Austin, L. S., \& Godleski, L. S. (1999). Therapeutic appraoches for survivors of disaster. Psychiatric Clinical of North America, 22, 897-910.

Badri, A., Crutzen, R., \& Van den Borne, H. W. (2012a). Experiences and psychosocial adjustment of Darfuri female students affected by war: An exploratory study. International Journal of Psychology, e-pub ahead of print.

Badri, A., Crutzen, R., \& Van den Borne, H. W. (2012b). Exposures to war-related traumatic events and post-traumatic stress disorder symptoms among displaced Darfuri female university students: An exploratory study. BMC Public Health, 12, 603.

Badri, A., Crutzen, R., \& Van den Borne, H. W. (submitted-a). Anxiety, depression and resilience among war-affected and non-war-affected Sudanese female university students: A comparative study.

Badri, A., Crutzen, R., \& Van den Borne, H. W. (submitted-b). Coping resources and resilience strategies among war-affected and non-war-affected Sudanese female university students: A comparative study.

Badri, A., Crutzen, R., Van den Borne, H. W., \& Eltayeb, S. (submitted-c). Promoting Darfuri women's psychosocial health: Developing a war-trauma counselor training program.

Badri, B. (2008). Sudanese women's profile and pathways to empowerment. Omdurman: Ahfad University for Women.

Basoglu, M., \& Mineka, S. (1992). The role of uncontrollability and unpredictability of stress in the development of post-torture stress symptoms. In M. Basoglu (Ed.), Torture and Its Consequences: Current Treatment Approaches: Cambridge University Press.

Basoglu, M., Salcioglu, E., \& Livanou, M. (2002). Traumatic stress responses in earthquake survivors in Turkey. Journal of Traumatic Stress, 15, 269-276.

Baumeister, R. F., \& Leary, M. R. (1995). The need to belong: Desire for interpersonal attachments as a fundamental human motivation. Psychological Bulletin, 117.

Becker, D. M., Hill, D. R., Jackson, J. S., Levine, D. M., Stillman, F. A., \& Weiss, S. M. (1992). Health behavior research in minority populations, (NIH publication No. 92-2965). Washington, DC: (Eds.). ()... .

Beiser, M. (1988). Influences of time, ethnicity, and attachment on depression in Southeast Asian refugees. American Journal of Psychiatry, 145, 46-51. 
Beiser, M., \& Hou, F. (2001). Language acqusition, unemployment and depressive disorder among Southeast Asian reugees: a 10-year study. Social Science and Medicine, 53, 1321-1334.

Beiser, M., \& Hyman, I. (1997). Refugees' time perspective and mental health. American Journal of Psychiatry, 154, 996-1002.

Benson, L. T., \& Deeter, T. E. (1992). Moderators of the relation between stress and depression in adolescents. School Counselor, 39, 189-194.

Berman, H., Giron, E., \& Marroquin, A. (2006). A narrative study of refugee women who have experienced violence in the context of war. Canadian Journal of Nursing Research, 38, 32-53.

Bertocci, D., Hirch, E., Sommer, W., \& Williams, A. (1992). Student mental health needs: Survey results and implications for service. Journal of American College Health, 43, 248-251.

Betancourt, T. S. (2005). Stressors, supports and the social ecology of displacement: psychosocial dimensions of an emergency education program for Chiechen adolescents displaced in Ingushetia, Russia. Culture, Medicine and Psychiatry, 29, 309-340.

Bichescu, D., Neuner, F., Schauer, M., \& Elbert, T. (2007). Narrative exposure therapy of political imprisonment-related chronic trauma-spectrum disorders. Behaviour Research and Therapy, 45, 2212-2220.

Bolton, P., Bass, J., Neugebauer, R., Verdeli, H., Clougherty, K. F., Wickramaratne, P., ... Weissman, M. M. (2003). Group interpersonal psychotherapy for depression in rural Uganda: a randomized controlled trial. JAMA, 289, 3117-3124.

Bramness, J. A., Fixdal, T. C., \& Vaglum, P. (1991). Effect of medical school stress on the mental health of medical students in early and late clinical curriculum. Acta Psychiatrica Scandinavica, 84, 340345.

Bramsen, I., \& Van der Ploeg, H. M. (1999). Fifty years later: The long-term psychological adjustment of ageing World War II survivors. Acta Psychiatrica Scandinavica, 100, 350-358.

Breslau, N., Davis, G. C., \& Andreeski, P. (1997). Sex differences in post-traumatic stress disorder. Archive General Psychiatry, 54.

Broadhead, J. C., \& Abas, A. M. (1997). Depression and anxiety among women in an urban setting in Zimbabwe. Psychological Medicine, 27, 59-71.

Bronfenbrenner, U. (1979). The ecology of human development: Experiments by nature and design. Cambridge, MA: Harvard University Press.

Bryant, R. A., \& Njenga, F. G. (2006). Cultural Sensitivity: Making trauma assessment and treatment plans culturally relevant. Journal of Clinical Psychiatry, 67, 74-79.

Cabrera, N. L., \& Padilla, A. M. (2004). Entering and succeeding in the "Culture of College": The story of two Mexican heritage students. . Hispanic Journal of Behavioral Sciences, 26, 152-170.

Campbell-Sills, L., Cohan, S. L., \& Stein, M. B. (2006). Relationship of resilience to personality, coping, and psychiatric symptoms in young adults. Behaviour Research and Therapy, 44, 585-599.

Cánive, J. M., \& Castillo, D. (1997). Hispanic Veterans Diagnosed with PTSD: Assessment and Treatment Issues. NCP Clinical Quarterly, 7, 12-15.

Carballo, M., Smajkic, A., Zeric, D., Dzidowska, M., Gerbre-Medhin, J., \& Van Halem, J. (2004). Mental health and coping in a war situation: The case of Bosnia and Herzegovina. Journal of Biosocial Science, $36,436-477$.

Cardozo, B. L., Bilukha, O. O., Crawford, C. A., Shaikh, I., Wolfe, M. I., \& Gerber, M. L. (2004). Mental health, social functioning and disability in postwar Afghanistan. JAMA, 292, 575-584.

Cardozo, B. L., Kaiser, R., Gotway, C. A., \& Agani, F. (2003). Mental health, social functioning, and feelings of hatred and revenge of Kosovar Albanians one year after the war in Kosovo. Journal of Traumatic Stress, 16, 351-360.

Cardozo, B. L., Vergara, A., Agani, F., \& Gotway, C. A. (2000). Mental health, social functioning, and attitudes of Kosovar Albanians following the war in Kosovo. JAMA, 284, 569-577.

Carr, P., Freund, K., \& Somani, S. (1995). The Medical Care of Women. Philadelphia, Pa: WB Saunders Co.

Ceja, M. (2004). Chicana college aspirations and the role of parents: Developing educational resiliency. Journal of Hispanic Higher Education, 3, 338-362.

Cepeda-Benito, A., \& Gleaves, D. H. (2000). Cross-ethnic equivalence of the Hopkins Symptom Checklist21 in European American, African American, and Latino college students. Cultural Diversity \& Ethnic Minority Psychology, 3, 297-308.

Christle, C. A., Harley, D., Nelson, C. M., \& Jones, K. (2007). Promoting resilience in children: What parents can do, Information for families. from Center for Effective Collaboration and Practice

Cohen, S. (1991). Stress during childhood and adolescence: Sources of risk and vulnerability. In E. M. Cummings, Greene, A. L., and Karraker, K. H. (Ed.), Life-Span Developmental Psychology: Perspectives on Stress and Coping. NJ: Erlbaum, Hillsdale. 
Coker, E. M. (2004a). Dislocated identity and the fragmented body: Discourses of resistance among southern Sudanese refugees in Cairo. Journal of Refugee Studies, 17, 401-419.

Coker, E. M. (2004b). 'Travelling Pains': Embodied metaphors of suffering among southern Sudanese refugees in Cairo. Culture, Medicine and Psychiatry, 28, 15-39.

Constantine, M. G., Chen, E. C., \& Ceesay, P. (1997). Intake concerns of racial and ethnic minority students at a university counselling center: Implications for developmental programming and outreach. Journal of Multicultural Counselling and Development, 25, 210-218.

Cope, R. G. (1972). Are students more likely to drop out of large colleges? College Student Journal, 6, 92-97.

D'Imperio, R. L., Dubow, E. F., \& Ippolito, M. F. (2000). Resilient and stress-affected adolescents in an urban setting. Journal of Clinical Child Psychology, 29, 129-142.

Dahlin, M., Joneborg, N., \& Runeson, B. (2005). Stress and depression among medical students: a crosssectional study. Medical Education, 39, 594-604.

Dalgard, O. S., Dowrick, C., \& Lehtinen, V. (2006). Negative life events, social support and gender difference in depression: a multinational community survey with data from the ODIN study. Social Psychiatry and Psychiatric Epidemiology, 41, 444-451.

Daly, A., Jennings, J., Beckett, J. O., \& Leashore, B. R. (1995). Effective coping-strategies of African Americans. Social Work, 40, 240-248.

De Jong, J. (2002). Trauma, war and violence: Public mental health in socio-cultural context. New York: Kluwer Academic/Plenum Publishers.

De Jong, J., Komproe, I. H., Ommeren, M. V., Masri, M. E., Araya, M., \& Khaled, N. (2001). Lifetime events and posttraumatic stress disorder in 4 postconflict settings. JAMA, 286, 555-562.

De Waal, A. (2005). Briefing: Darfur, Sudan - Prospects for Peace. African Affairs, 104127 - 135.

De Waal, A. (2009). Tragedy in Darfur: On understanding and ending the horror Retrieved May 20, 2010, from http://bostonreview.net/BR29.5/dewaal.html

Derogatis, L. R., Lipman, R. S., Rickels, E. H., Uhlenhuth, E. H., \& Covi, L. (1974). SCL-90-R: An outpatient psychiatric rating scale. Psychopharmacology Bulletin, 9, 13-28.

Dodani, S., \& Zuberi, W. R. (2000). Centre-based prevalence of anxiety and depression in women of the Northern areas of Pakistan. Journal of Pakistan Medical Association, 50, 138-40.

Driscoll, A. K. (2006). Academic resilience among low SES high school students. Paper presented at the Population Association of America, Los Angeles, CA.

Drozdek, B. (2003). Chronic PTSD and medical services utilization by asylum seekers. Journal of Refugee Studies, 16, 201-211.

DuBois, D. L., Felner, R. D., Brand, S., Adan, A. M., \& Evans, E. G. (1992). A prospective study of life stress, social support, and adaptation in early adolescence. Child Development, 63, 542-557.

Dubow, E. F., Edwards, S., \& Ippolito, M. F. (1997). Life stressors, neighborhoods, disadvantage, and resources: A focus on inner-city children's adjustment. Journal of Clinical Child Psychology, 26, 130-144.

Duru, E., \& Poyrazli, S. (2007). Personality dimensions, psychosocial-demographic variables, and English language competency in predicting level of acculturative stress among Turkish international students. International Journal of Stress Management, 14, 99-110.

Ehlers, A., \& Clark, D. M. (2000). A cognitive model of posttraumatic stress disorder. Behaviour Research and Therapy, 38, 319-345.

Eisenbruch, M. (1991). From post-trauma stress disorder to 'cultural bereavement': Diagnosis of Southeast Asian refugees. Social Science and Medicine, 33.

Elder, G. H. (1998). The life course as developmental theory. Journal of Child Development, 69.

Elnur, I. (1994, 5-9 January 1994). Displaced and refugee studies in the Sudan: Present trends and future prospects. Paper presented at the 4th International Research and Advisory panel conference, Summerville College, University of Oxford.

Farhood, L., Zurayk, H., Chaya, M., Saadeh, F., Meshefedjian, G., \& Sedani, T. (1993). The impact of war on the physical and mental health of the family: The Lebanese experience. Social Science \& Medicine, 36, 1555-1567.

Farwell, N. (2001). "Onward through strength": Coping and psychological support among refugee youth returning to Eritrea from Sudan. Journal of Refugee Studies, 14, 43-69.

Fawazi, M. C., Pham, T., Lin, L., Nguyen, T. V., Ngo, D., Murphy, E., \& Mollica, R. F. (1997). The validity of post-traumatic stress disorder among Vietnamese refugees. Journal of Trauma Stress, 10, 101108.

Fazel, M., \& Stein, A. (2003). Mental health of refugee children: comparative study. British Medical Journal $327,134$. 
Ferren, P. M. (1999). Comparing perceived self- efficacy among adolescent Bosnian and Croatian refugees with and without posttraumatic stress disorder. Journal of Traumatic Stress, 12, 405-420.

Finch-Shakespeare, J., \& Wickham, K. (2009). Adaptation of Sudanese refugees in an Australian context: Investigating helps and hindrances. International Migration, 48, 23-46.

Flaherty, J. A., Gaviria, F. M., Pathak, D., Mitchell, T., Wintrob, R., Richman, J. A., \& Birz, S. (1988). Developing Instruments for Cross-Cultural Psychiatric Research. The Journal of Nervous and Mental Disease, 176, 257-263.

Foa, E., Keane, T., \& Friedman, M. (2000). Effective treatments for PTSD. London: Guilford Press.

Folkman, S. (1997). Positive psychological states and coping with severe stress. Social Science and Medicine, 45, 1207-1221.

Folkman, S., Moskowitz, J. T., Ozer, E. M., \& Park, C. L. (1997). Positive meaniningful events and coping in the context of HIV/AIDS. In B. H. Gottlieb (Ed.), Coping with chronic stress (pp. 293-314). New York: Plenum Press.

Fox, S. H., \& Tang, S. S. (2000). The Sierra Leonean refugee experience: Traumatic events and psychiatric sequelae. Journal of Nervous and Mental Disease, 188, 490-495.

Gallo, L. C., \& Matthews, K. A. (2003). Understanding the association between socioeconomic status and physical health: Do negative emotions play a role? . Psychological Bulletin, 129, 10-51.

Gan, Y., Zhang, Y., Wang, X., Wang, S., \& Shen, X. (2006). The coping flexibility of neurasthenia and depressive patients. Journal of Personality and Individual Differences, 40, 859-871.

Garmezy, N. (1991). Resilience and vulnerability to adverse developmental outcomes associated with poverty. American Behavioral Scientist, 34, 416-430.

Garmezy, N., \& Tellegen, A. (1984). Studies of stress-resistent children: methods, variables and preliminary findings. In F. Morrison, D. Keating \& C. Lord (Eds.), Applied Developmental Psychology. New York: Academic Press.

Gavrilovic, J., Lecic-tosevski, D., Knezevic, G., \& Priebe, S. (2002). Predictors of posttraumatic stress in civilians 1 year after air attacks: A study of Yugoslavian students. The Journal of Nervous and Mental Disease, 190, 257-262.

Geltman, P. L., Grant-Knight, W., Mehta, S. D., Lloyd-Travaglini, C., Lustig, S., Landgraf, J. M., \& Wise, P. H. (2005). The 'lost boys of Sudan': functional and behavioral health of unaccompanied minors resettled in the United States. Archives of Pediatrics and Adolescent Medicine, 159, 585-591.

Ghorashi, H. (2008). Giving silence a chance: The importance of life stories for research on refugees. Journal of Refugee Studies, 21, 117-132.

Gillespie, M., \& Noble, J. (1992). Iowa City, IA Patent No. American College Testing Program.

Gloria, A. M. (1997). Chicana academic persistence: Creating a university-based community. Education \& Urban Society, 30, 107-121.

Gloria, A. M., Castellanos, J., \& Orozco, V. (2005). Perceived educational barriers, cultural fit, coping responses, and psychological well-being of Latina undergraduates. Hispanic Journal of Behavioral Sciences, 27, 161-183.

Good, B. J., \& Kleinman, A. M. (1985). Culture and anxiety: Cross-cultural evidence for the patterning of anxiety disorders Anxiety and the anxiety disorders (pp. 297-323). Hillsdale, NJ: Lawrence Erlbaum Associate.

Goodman, J. H. (2004). Coping with trauma and hardships among unaccompanied refugee youths from Sudan. Qualitative Health Research, 14, 1177-1196.

Green, B. L. (1996). Traumatic stress and disaster: Mental health effects and factors influencing adaptation. In F. L. Mak, \& C. C. Nadelson (Eds.), International review of psychiatry. Washington, DC: American Psychiatric Press.

Greenberg, M. T., Lengua, L. J., Coie, J. D., \& Pinderhughes, E. E. (1999). Predicting developmental outcomes at school entry using a multiple-risk model: Four American communities. Journal of Developmental Psychology, 35, 403-417.

Greenland, K., \& Brown, R. (2005). Acculturation and contact in Japanese students studying in the United Kingdom. Journal of Social Psychology, 145, 373-389.

Guillemin, F., Bombardier, C., \& Beaton, D. (1993). Cross cultural adaptations of health related quality of life measures: literature review of proposed guidelines. Journal of Clinical Epidemiology, 46, 1417-1432.

Hackett, G., Betz, N. E., Casas, J. M., \& Rocha-Singh, I. A. (1992). Gender, ethnicity, and social cognitive factors predicting the academic achievement of students in engineering. Journal of Counseling Psychology, 4, 527-538. 
Halepota, A. A., \& Wasif, S. A. (2001). Harvard Trauma Questionnaire Urdu translation: the only cross culturally validated screening instrument for the assessment of trauma and torture and their sequelae. Journal of Pakistani Medical Association, 51, 285-290.

Hall, C. W., Spruill, K. L., \& Webster, R. E. (2002). Motivational and attitudinal factors in college students with and without learning disabilities. Learning Disability Quarterly, 25, 79-86.

Hamad, B. (1974). Some home factors in relation to mental health in Khartoum University students. Journal of the American College Health Association, 23, 127-133.

Hamid, A. A. R. M., \& Musa, S. A. (2010). Mental health problems among internally displaced persons in Darfur. [Article]. International Journal of Psychology, 45, 278-285.

Haro, R. P., Rodriguez, G. J., \& Gonzales, L. J. (1994). Latino persistence in higher education: A 1994 survey of University of California and California State University Chicano/Latino students.

Harpham, T. (1994). Urbanization and mental health in developing countries: a research role for social scientists, public health professionals and social psychiatrists. Social Science and Medicine, 39, 223-245.

Henderson, S., Byrne, D. G., \& Duncan-Jones, P. (1981). Neurosis and Social Environement. Sydney: Academic Press.

Herman, J. L., Russell, D. E., \& Trocki, K. (1986). Long-term effects of incestuous abuse in childhood. American Journal of Psychiatry, 143, 1293-1296.

Hesbacher, P. T., Rickels, K., Morris, R. J., Newman, H., \& Rosenfeld, H. (1980). Psychiatric illness in family practice. Journal of Clinical Psychiatry, 41, 6-10.

Higgins, G. O. (1994). Resilient Adults: Overcoming a Cruel Past. San Francisco, Calif: Jossey-Bass.

Hinton, D., Pham, T., \& Minh, T. (2004). CBT for Vietnamese refugees with treatment-resistant PTSD and panic attacks: a pilot study. Journal of Traumatic Stress, 17, 429-433.

Hobfoll, S. (1991). Traumatic stress: A theory based on rapid loss of resources. Anxiety, Stress \& Coping: An International Journal, 4, 187-197.

Hobfoll, S. E. (1989). Conservation of resources: A new attempt at conceptualizing stress. American Psychologist, 44, 13-524.

Holleran, L. K., \& Waller, M. A. (2003). Sources of resilience among Chicano/a youth: Forging identities in the borderlands. Child and Adolescent Social Work Journal, 20, 335-350.

Howard, J. M., \& Goelitz, A. (2004). Psychoeducation as a response to community disaster. Brief Treat Crisis Intervention, 4, 1-10.

Hysenbegasi, A., Hass, S. L., \& Rowland, C. R. T. (2005). The impact of depression on the academic productivity of university students. Journal of Mental Health Policy and Economics, 8, 145-151.

Igreja, V., Kleijn, A., \& Richters, A. (2006). When the war was over, little changed. Women's posttraumatic suffering after the war in Mozambique. Journal of Nervous and Mental Disease, 194, 502-509.

Inter-Agency Standing Committee. (2007). IASC Guidelines on Mental Health and Psychosocial Support in Emergency Settings. Geneva: IASC.

Iwasaki, Y. (2003). Roles of leisure in coping with stress among university students: A repeatedassessment field study. Anxiety, Stress \& Coping: An International Journal, 16, 31- 57.

Johnson, D., Polusny, M. A., Erbes, C. R., King, D., King, L., Litz, B. T., . . Southwick, S. M. (2009). Resilience and response to stress: Development and initial validation of the Response to Stressful Experiences Scale (RSES). Journal of Affective Disorders.

Jones, P. S., Lee, J. W., \& Phillips, L. R. (2001). An adaptation of Brislin's translation model for crosscultural research. Nursing Research, 50, 300-304.

Joseph, M., Chipandambira, K. S., Hungwe, C., \& Mupawose, A. (2007). Stressors and coping strategies of state university students in a developing country Kasayira. Journal of Psychology in Africa Community psychology:Special issue, 17, 45-50.

Joyce, A., Earnest, J., De Mori, G., \& Silvagni, G. (2010). The experinece of students from refugee backgrounds at universities in Australia: Reflections on the social, emotional, and practical challenges. Journal of Refugees Studies, 23.

Kagee, A. (2005). Symptoms of distress and posstraumatic stress among South African former political detainees. Ethnicity and health, 10, 169-179.

Kaplan, I. (2009). Effects of trauma and the refugee experience on psychological seessment process and interpretation. Austalian Psychologist: Issues in cross-cultural psychological assessment, 44, 6-15.

Karunakara, U., Neuner, F., Schauer, M., Singh, K., Hill, K., Elbert, T., \& Burnham, G. (2004). Traumatic events and symptoms of post-traumatic stress disorder amongst Sudanese nationals, refugees and Ugandans in the West Nile. African Health Science, 4, 83-93.

Katerndahl, D. A., \& Parchman, M. (2002). The ability of the stress process model to explain mental health outcomes. Comprehensive Psychiatry, 34, 351-360. 
Kelly, J. G., Ryan, A. M., Altman, B. E., \& Stelzner, S. P. (1993). Understanding and changing social systems: An ecological view. In E. Seidman, and Rappaport, J. (Ed.), Handbook of Community Psychology. New York: Plenum Press.

Kessler, R. C., Sonnega, A., Bromet, E., Hughes, M., \& Nelson, C. M. (1995). Posttraumatic stress disorder in the National comorbidity survey. Archives of General Psychiatry, 52.

Khawaja, N. G., White, K. M., Schweitzer, R., \& Greenslade, J. (2008). Difficulties and coping strategies of Sudanese refugees: A qualitative approach. Transcultural Psychiatry, 45, 489-512.

Khouzam, H. R., \& Kissmeyer, P. (1997). Antidepressant treatment, posttraumatic stress disorder, and survivor guilt, and spiritual awakening. Journal of Trauma Stress, 10, 691-696.

Kim, G., Torbay, R., \& Lawry, L. (2007). Basic health, women's health, and mental health among internally displaced persons in Nyala province, south Darfur, Sudan. American Journal of Public Health, 97, 353-361.

Kinderman, P., \& Bentall, R. (1997). Attributional therapy for paranoid delusions: A case study. Behavioural and Cognitive Psychotherapy, 25, 269-280.

Kinzie, J. D., Boehnlein, J. K., Leung, P. K., Moore, L. J., Riley, C., \& Smith, D. (1990). The prevalence of posttraumatic stress disorder and its clinical significance amongst Southeast Asian refugees. American Journal of Psychiatry, 147, 913-917.

Kleijn, W. C., Hovens, J. E., \& Rodenburg, J. J. (2001). Post-traumatic stress symptoms in refugees: assessments with the Harvard Trauma Questionnaire and the Hopkins symptom Checklist-25 in different languages. Psychological Reports, 88, 527-532.

Klerman, G. L., Weissman, M. M., Rounsaville, B. J., \& Chevron, E. S. (1984). Interpersonal Psychotherapy of Depression. New York: Basic Books.

Knight, Z. G. (2006). The Trauma and beyond trauma of political displacement: An exploratory case study of one Zimbabwean farmer. The Australian Journal of Disaster and Trauma, 1.

Krishnan, K. U., Frank, N., Schauer, M., Singh, K., Kenneth, H., Elbert, T., \& Burnham, G. (2004). Traumatic events and symptoms of post-traumatic stress disorder amongst Sudanese nationals, refugees and Ugandans in the West Nile. African Health Sciences, 4, 83-93.

Kuo, C. J., Tang, H. S., \& Tsay, C. J. (2003). Prevalence of psychiatric disorders among bereaved survivors of a disastrous earthquake in Taiwan. Pyschiatric Servivces, 54, 249-251.

Lazarus, R. S. (1993). Coping theory and research: Past, present and future. Psychometric medicine, 55, 237-247.

Lazarus, R. S., \& Folkman, S. (1984). Stress, appraisal, and coping. New York: Springer.

Lee, Y., Lee, M. K., Chun, K. H., Lee, Y. K., \& Yoon, S. J. (2001). Trauma experience of North Korean refguees in China. American Journal Prev Medicine, 20, 225-229.

Leong, F. T. L., Wagner, N. S., \& Tata, S. P. (1995). Racial and ethnic variations in helping-seeking attitudes. Thousand Oaks, CA: Sage Publications.

Lerner, M. J., \& Gignac, M. A. M. (1992). Is it coping or is it growth? A cognitive-affective model of contentment in the elderly. In S. F. L. Montada, and M. J. Lerner (Ed.), Life crises and experiences of loss in adulthood (pp. 321-337). Hilsdale, NJ: Erlbaum.

Lester, D., \& DeSimone, A. (1995). Depression and suicidal ideation in African American and Caucasian students. Psychological Reports, 77.

Licitra-Kleckler, D. M., \& Waas, G. A. (1993). Perceived social support among high-stress adolescents: The role of peers and family. Journal of Adolescence Research, 8, 381-402.

Lie, B. (2002). A three year foolow-up study of psychosocial functioning and general symtoms in settled refugees. Acta Psychiatrica Scandinavica, 106, 415-425.

Lundberg, P., Cantor-Graae, E., Rukundo, G., Ashaba, S., \& Östergren, P. (2009). Urbanicity of place of birth and symptoms of psychosis depression and anxiety in Uganda. British Journal of Psychiatry, 195, 156-162.

Luthar, S. S. (1991). Vulnerability and resilience: A study of high risk adolescents. Journal of Child Development, 62, 600-616.

Luthar, S. S., \& Cicchetti, D. (2000). The construct of resilience: Implications for interventions and social policies. Development and Psychopathology, 12, 857-885.

Luthar, S. S., Cicchetti, D., \& Becker, B. (2000). The construct of resilience: A critical evaluation and guidelines for future work. Journal of Child Development, 71, 543-562.

Macksoud, M. S., \& Aber, J. L. (1996). The war experiences and psychosocial development of children in Lebanon. Child Development, 67, 70-88.

Majka, L., \& Mullan, B. (1992). Employment retention, area of origin and type of social support among refugees in the Chicago area. . International Migration Review, 26, 899-926. 
Maltzan, T. L. (2006). Rurality and higher education: Implications for identity and persistence. Unpublished doctoral dissertation, The Ohio State University.

Marks, I., Lovell, K., Noshirvani, H., Livanou, M., \& Thrasher, S. (1998). Treatment of posttraumatic stress disorder by exposure and/or cognitive restructuring: a controlled study. Archives of General Psychiatry, 55, 317-325.

Marlowe, J. M. (2010). Beyond the discourse of trauma: Shifting the focus on Sudanese refugees. Journal of Refugees Studies, 23, 183-198.

Maslow, A. H. (1943). A theory of human motivation. Psychological Review, 50, 370-396.

Masten, A. S., Best, K. M., \& Garmezy, N. (1990). Resilience and development: Contributions from the study of children who overcome adversity. Development and Psychopathology, 2, 425-444.

Masten, A. S., \& Curtis, W. J. (2000). Integrating competence and psychopathology: Pathways towards a comprehensive science of adaptation in development. Development and Psychopathology, 12, 529-550.

Masten, R., Tušak, M., Zalar, B., \& Ziherl, S. (2009). Stress, coping and social support in three groups of university students. Psychiatria Danubina, 21, 41-48.

Matheny, K. B., Curlette, W. L., Aysan, F., Herrington, A., Gfroerer, C. A., Thompson, D., \& Hamarat, E. (2002). Coping resources, perceived stress, life satisfaction among Turkish and American university students. International Journal of Stress Management, 9, 81-97.

Mays, N., \& Pope, C. (1995). Rigour and qualitative research. British Medical Journal, 311, 109-112.

McIntire, W. G., Marion, S. F., \& Quaglia, R. (1990). Rural school counselors: Their communities and schools. The School Counselor, 37, 166-172.

Meffert, S. M., \& Marmar, C. R. (2009). Darfur refugees in Cairo: Mental health and interpersonal conflict in the aftermath of genocide. Journal of Interpersonal Violence, 24, 1835-1848.

Mena, F. J., Padilla, A. M., \& Maldonado, M. (1987). Acculturative stress and specific coping strategies among immigrant and later generation college students. Hispanic Journal of Behavioral Sciences, 9, 207-225.

Miller, D. B. (1999). Racial socialization and racial identity: Can they promote resiliency for African American adolescents? Adolescence, 34, 493-501.

Miller, K., Weine, S., Ramic, A., Brkic, N., Djuric Bjedic, Z., Smajkic, A. (2002). The relative contribution of war experiences and exile-related stressors to levels of psychological distress among Bosnian Refugees. Journal of Traumatic Stress, 15, 377-387.

Miller, K. E., Omidian, P., Rusmussen, A., Yaqubi, A., \& Daudzai, H. (2008). Daily stressors, war experiences, and mental health in Afghanistan. Transcultural Psychiatry, 45, 611-638.

Miller, K. E., \& Rasmussen, A. (2010). War exposure, daily stressors, and mental health in conflict and post-conflict settings: Bridging the divide between trauma-focused and psychosocial frameworks. Social Science \& Medicine, 70, 7-16.

Misra, R., \& Castillo, L. G. (2004). Academic Stress Among College Students Academic Stress Among College Students: Comparison of American and International Students. International Journal of Stress Management, 11, 132-148.

Moffat, K. J., McConnachie, A., Ross, S., \& Morrison, J. M. (2004). First year medical student stress and coping in a problem-based learning medical curriculum. Medical Education, 38, 482-491.

Moisander, P. A., \& Edston, E. (2003). Torture and its sequel-A comparison between victims from six countries. Forensic Science International, 137, 133-140.

Mollica, R., McDonald, L., Massagli, M., \& Silove, D. (2004). Measuring trauma, measuring torture: Instructions and guidelines on the utilization of the Harvard Program in Refugee Trauma's Versions of the Hopkins Symptom Checklist-25 (HSCL-25) and the Harvard Trauma Questionnaire (HTQ). Cambridge: MA: Harvard Program in Refugee Trauma.

Mollica, R., McInnes, K., Pham, T., Smith, M. C., Murphy, E., \& Lin, L. (1998). The dose-effect relationships between torture and psychiatric symptoms in Vietnamese ex-political detainees and a comparison group. Journal of Nervous and Mental Disease, 186, 542-553.

Mollica, R., Poole, C., Son, L., \& Murray, C. (1997). Effects of war trauma on Cambodian refugee adolescents' functional health and mental health status. Journal of the American Academy of Child \& Adolescent Psychiatry, 36, 1098-1106.

Mollica, R. F., Caspi-Yavin, Y., Bollini, P., Truong, T., Tor, S., \& Lavell, J. (1992). The Harvard Trauma Questionnaire: Validating a cross-cultural instrument for measuring torture, trauma, and posttraumatic stress disorder in Indochinese refugees. Journal of Nervous and Mental Disease, $180,111-116$ 
Mollica, R. F., Caspi-Yavin, Y., Lavelle, J., Tor, S., Yang, T., Chan, S., . . de Marneffe, D. (1995). The Harvard Trauma Questionnaire (HTQ) Manual: Cambodian, Loatian, and Vietnamese versions. Torture, Suppl 1, 19-42.

Mollica, R. F., Donelan, K., Tor, S., \& al., e. (1993). The effect of trauma and confinement on functional health and mental health status of Cambodians living in Thailand Cambodia border camps. JAMA, 270, 581-586.

Mollica, R. F., Sarajlic, N., Chernoff, M., Lavelle, J., Vukovic, I. S., \& Massagli, M. P. (2001). Longitudinal study of psychiatric symptoms, disability, mortality, and emigration among Bosnian refugees. JAMA, 286, 546-554.

Mollica, R. F., Wyshak, G., de Marneffe, D., Khoun, F., \& Lavelle, J. (1987). Indochinese versions of the Hopkins Symptom Checklist-25: A screening instrument for the psychiatric care of refugees. American Journal of Psychiatry, 144, 479-500.

Momartin, S., Silove, D., Manicaasagar, V., \& Steel, Z. (2004). Comorbidity of PTSD and Depression: Association with Trauma Exposure, Symptom Severity and Functional Impairment in Bosnian Refugees Resettled in Australia. Journal of Affective Disorders, 80, 231-238.

Morgos, D., Worden, J. W., \& Gupta, L. (2008). Psychosocial effects of war experiences among displaced children in southern Darfur. Journal of Death and Dying, 56, 229-253.

Moritsugu, J. N., \& Sue, S. (1983). Minority status as a stressor. New York: Pergamon.

Mufson, L., Gallagher, T., Pollack Dorta, K., \& Young, J. F. (2004). A group adaptation of Interpersonal Psychotherapy for depressed adolescents. American Journal of Psychotherapy, 58, 220-237.

Mufson, L., Pollack, D. K., Wickramaratne, P., Nomura, Y., Olfson, M., \& Weissman, M. M. (2004). A randomized effectiveness trial of interpersonal psychotherapy for depressed adolescents. Archives of General Psychiatry, 61, 577-584.

Mufson, L., Weissman, M. M., Moreau, D., \& Garfinkel, R. (1999). Efficacy of interpersonal psychotherapy for depressed adolescents. Archives of General Psychiatry, 56, 573-579.

Mumford, D. B., Minhas, F. A., Akhtar, I., \& et. al. (2000). Stress and psychiatric disorder in urban Rawalpindi: community survey British Journal of Psychiatry, 177, 557-562.

Murray, K., Davidson, G., \& Schweitzer, R. (2008). Psychological Well-being of Regugees Resettling in Australia (pp. 1-28). Melbourne: The Austalian Psychological Society.

Nagase, Y., Uchiyama, M., Kaneita, Y., Li, L., Kaji, T., Takahashi, S., . . . Ohida, T. (2009). Coping strategies and their correlates with depression in the Japanese general population. Psychiatric Research, 168, 57-66.

Neuner, F., Onyut, P., Ertl, V., Schauer, E., Odenwald, M., \& Elbert, T. (2008). Treatment of posttraumatic stress disorder by trained lay counsellors in an African refugee settlement - a randomized controlled dissemination trial. Journal of Consulting and Clinical Psychology, 76, 686-694.

Neuner, F., Schauer, M., Elbert, T., \& Roth, W. T. (2002). A narrative exposure treatment as intervention in a refugee camp: a case report. Behavioral and Cognitive Psychotherapy, 30, 205-209.

Neuner, F., Schauer, M., Karunakara, U., Klaschik, C., Robert, C., \& Elbert, T. (2004a). Psychological trauma and evidence for enhanced vulnerability for posttraumatic stress disorder through previous trauma among West Nile refugees. BMC Psychiatry, 4, 34-40.

Neuner, F., Schauer, M., Klaschik, C., Karunakara, U., \& Elbert, T. (2004b). A comparison of narrative exposure therapy, supportive counseling, and psychoeducation for treating posttraumatic stress disorder in an African refugee settlement. Journal of Consulting and Clinical Psychology, 72, 579587.

Newman, C. J. (1976). Disater at Buffalo creek, children of disaster: clinical obsercations at Buffalo creek. American Journal of Psychiatry, 133, 306-312.

Nilsson, D. (2000). Internally displaced, Refugees and Returness from and in the Sudan: A Review. Studies of Emergencies and Disaster Relief, 8.

OCHA. (1999). Handbook for applying the Guiding Principles on Internal Displacement Office for Coordination of Humanitarian Affairs. United Nations, New York: The Brookings Institute Project on Internal Displacement, Office for the Coordination of Humanitarian Affairs.

OCHA. (2006). Darfur Humanitarian Profile No. 25 - 01 October 2006. In O. UN Darfur Coordination and Information Cell (Ed.), Resident and Humanitarian Co-ordinator UN (pp. 1-11). Khartoum: Office of UN Deputy Special Representative of the UN Secretary-General for Sudan.

Ogbu, J. U. (1992). Understanding cultural diversity and learning. Educational Researcher, 21, 5- 14.

Onyut, P. L., Neuner, F., Schauer, E., Ertl, V., Odenwald, M., Schauer, M., \& Elbert, T. (2004). The Nakivale Camp Mental Health Project: building local competency for psychological assistance to traumatised refugees. Intervention, 2, 90-107. 
Onyut, P. L., Neuner, F., Schauer, E., Ertl, V., Odenwald, M., Schauer, M., \& Elbert, T. (2005). Narrative exposure therapy as a treatment for child war survivors with posttraumatic stress disorder: two case reports and a pilot study in an African refugee settlement. BMC Psychiatry, 5, 7.

Ostrom, T. M. (1984). The sovereignty of social cognition. In R. S. W. T. K. Srull (Ed.), Handbook of social cognition (vol. 1, pp. 1-37). Hillsdale, NJ: Erlbaum.

Otto, M. W., Hinton, D., \& Korbly, N. B. (2003). Treatment of pharmacotherapy-refactory posttraumatic stress disorder among Cambodian refugees: a pilot study of combination treatment with cognitive-behavior therapy. Behavior Research Therapy, 41, 1271-1276.

Paardekooper, B., De Jong, J. T., \& Hermanns, J. M. (1999). The psychological impact of war and the refugee situation on south Sudanese children in refugee camps in northern Uganda: An exploratory study. Journal of Child Psychology and Psychiatry, 40, 529-536.

Palmieri, P. A., Galea, S., Canetti-Nisim, D., Johnson, R. J., \& Hobfoll, S. E. (2008). The Psychological Impact of the Israel-Hezbollah War on Jews and Arabs in Israel: The Impact of Risk and Resilience Factors. Social Science and Medicine, 67, 1208-1216.

Pan, J., Wong, D. F., Chan, C. L., \& Joubert, L. (2008). Meaning of life as a protective factor of positive affect in acculturation: A resilience framework and a cross-cultural comparison. International Journal of Intercultural Relations, 32, 505-514.

Papadopoulos, R. K. (2001a). Refugee families: Issues of systemic supervision. Journal of Family Therapy, $23,405-422$.

Papadopoulos, R. K. (2001b). Refugees, therapists and trauma: systemic reflections. Association for Family Therapy, Special issue on Refugees, 5-8.

Papadopoulos, R. K. (2002). Refugees, home and trauma. In R. K. Papadopoulos (Ed.), Therapeutic Care for Refugees. No Place Like Home (pp. 9-40). London: Karnac.

Papadopoulos, R. K. (2004). Trauma in a Systemic Perspective; theoretical, organisational and clinical dimensions. Paper presented at the Congress of the International Family Therapy Association XIV Istanbul.

Papadopoulos, R. K. (2007). Refugees, trauma, and Adversity-Activated Development. European Journal of Psychopathology and Counselling, 9, 301-312.

Papadopoulos, R. K., \& Hildebrand, J. (1997). Is Home Where The Heart Is? Narratives Of Oppositional Discourses in Refugee Families. In R. P. J. Byng-Hall (Ed.), In Multiple Voices: Narrative in Systemic Family Psychotherapy (pp. 206-236). London: Duckworth.

Parker, C. L., \& Folkman, S. (1997). Meaning in the context of stress. Review of General Psychology, 1, 115144.

Parsons, J. W. (1992). Undergraduate persistence rates of rural Californians at the University of California, Berkeley. Unpublished dissertation. University of California, Berkeley.

Paukert, A. L., Pettit, J. W., Perez, M., \& Walker, R. L. (2006). Affective and attributional features of acculturative stress among ethnic minority college students. The Journal of Psychology, 140, 405419.

Pearlin, L., \& Schooler, C. (1978). The structure of coping. Journal of Health and Social Behaviour, $19,2-21$.

Pearlin, L. I. (1989). The sociological study of stress. Journal of Health and Social Behavior, 30, 241-256.

Pearlin, L. I. (1999). The stress process revisited: Reflections on concepts and their interrelationships. In C. S. Aneshensel \& J. C. Phelan (Eds.), Handbook of the Sociology of Mental Health (pp. 395-415). New York: Kluwer Academic/Plenum Publishers.

Pearlin, L. I., Menaghan, E. G., Lieberman, M. A., \& Mullan, J. T. (1981). The stress process. Journal of Health and Social Behavior, 22, 337-356.

Pearson, R. E., \& Sutton, J. M. (1999). Rural and small town school counselors. Journal of Research in Rural Education, 15, 90-100.

Peltzer, K. (1999). Trauma and mental health problems of Sudanese refugees in Uganda. Central African Journal of Medicine, 45, 110-113.

Perkonigg, A., Kessler, R., \& Storz, S. (2000). Traumatic events and posttraumatic stress disorder in the community: prevalence, risk factors and comorbidity. Acta Psychiatrica Scandinavica, 101, 46-59.

Phinney, J. S., \& Alipura, L. L. (1990). Ethnic identity in college students from four ethnic groups. Journal of Adolescence, 13, 171-183.

Porter, M., \& Haslam, N. (2005). Pre-displacement and post-displacement factors associated with mantal health of refugees and internally displaced persons. Journal of the American Medical Association, 294, 602-612.

Rasmussen, A., \& Annan, J. (2009). Predicting stress related to basic needs and safety in Darfur refugee camps: A structural and social ecological analysis. Journal of Refugee Studies, 23. 
Rasmussen, A., Nguyen, L., Wilkinson, J., Raghavan, S., Vundla, S., Miller, K. E., \& Keller, A. S. (2010). Rates and impact of trauma and current stressors among Darfuri refugees in eastern Chad. American Journal of Orthopsychiatry, 80, 227-236.

Rasmussen, A., Smith, H., \& Keller, A. S. (2007). Factor structor of PTSD, symptoms amongst west and central African refugees. Journal of Traumatic Stress, 20, 271-280.

Resick, P. A., Nishith, M., Weaver, T. L., Astin, M. C., \& Feuer, C. A. (2002). A comparison of cognitiveprocessing therapy with prolonged exposure and a waiting condition for the treatment of chronic posttraumatic stress disorder in female rape victims. Journal of Consulting and Clinical Psychology, 70, 867-879.

Rew, L., Taylor-Seehafer, M., \& Thomas, N. Y. (2001). Correlates of resilience in homeless adolescents. Journal of Nursing Scholarship, 33, 33-40.

Rice, K. G., Herman, M. A., \& Petersen, A. C. (1993). Coping with challenge in adolescence: A conceptual model and psycho-educational intervention. Journal of Adolescence, 16, 233-251.

Roberts, B., Damunda, E. Y., Lomoro, O., \& Sondrop, E. (2009a). Post-conflict mental health needs: A crosssectional survey of trauma, depression and associated factors in Juba, Southern Sudan. British Medical Consulting BMC and Psychiatry, 9, 7.

Roberts, B., Damunda, E. Y., Lomoro, O., \& Sondrop, E. (2010). The influence of demographic characteristics, living conditions, trauma exposure on the overall health of a conflict-affected population in Southern Sudan. BMC Public Health, 10.

Roberts, B., Ocaka, K. F., Browne, J., Oyok, T., \& Sondrop, E. (2009b). Factors associated with the health status of internally displaced persons in northern Uganda. Journal of Epidemiological Community Health, 63, 227-232.

Robertson, M., Rushton, P. J., Bartrum, D., \& Ray, R. (2004). Group-based interpersonal psychotherapy for posttraumatic stress disorder: Theoretical and clinical aspects. International Journal of Group Psychotherapy, 54, 145-175.

Rogers, K. L., \& Leydesdorff, S. (2004). Trauma:life stories of survivors. New Brunswick, NJ: Transaction Publishers.

Rosenthal, J. A. (1996). Qualitative descriptors of strength of association and effect size. Journal of Social Science Research, 21.

Roth, S., Wayland, K., \& Woolsey, M. (1990). Victimization history and victim assailant relationship as factors in recovery from sexual assault. Journal of Traumatic Stress, 3, 169-180.

Runtz, M. G., \& Schallow, J. R. (1997). Social support and coping strategies as mediators of adult adjustment following childhood maltreatment. Child Abuse and Neglect, 21, 211-226.

Rutter, M. (1981). Stress, coping and development: some issues and some questions. Journal of Child Psychology and Psychiatry, 22, 323-256.

Rutter, M. (1985). Resilience in the face of adversity: protective factors and resistance to psychiatric disorder. British Journal of Psychiatry, 147, 598-611.

Saipanish, R. (2003). Stress among medical students in a Thai medical school. Medical Teacher, 25, 502506.

Saleebey, D. (1997). The strengths perspective in social work practice. New York: Longman.

Sandhu, D. S., \& Asrabadi, B. R. (1994). Development of an acculturative stress scale for international students: Preliminary findings. Psychological Reports, 435-448.

Sandhu, D. S., \& Asrabadi, B. R. (1998). An acculturative stress scale for international students. A practical approach to stress management. In C. P. Zalaquette \& R. J. Wood (Eds.), Evaluating stress: A book of resources (Vol. 2, pp. 1-33). Lanham, MD: Scarecrow Press.

Sapir, D. G. (1993). Natural and man-made disaters: The vulnerablity of women-headed households and children without families. World Health Statistics Quarterly, 46, 227-233.

Sarason, I. G., Sarason, B. R., \& Pierce, G. R. (1994). Social support : Global and relationship-based levels of analysis. Journal of Social and Personal Relationships, 11, 295-312.

Sarraj, E. E., Punamaki, R. L., Salmi, S., \& Summerfield, D. (1996). Experiences of torture and ill-treatment and psottraumatic stress disorder symptoms among Palestinian political prisoners. Journal of Traumatic Stress, 9, 595-606.

Scarr, S., \& McCartney, K. (1983). How people make their own environments: A theory of genotypeenvironement effects. Journal of Child Development, 54, 424-435.

Schaal, S., Elbert, T., \& Nuener, F. (2009). Narrative Exposure Therapy versus Interpersonal Psychotherapy: A Pilot Randomized Controlled Trial with Rwandan Genocide Orphans. Psychother Psychosom, 78, 298-306.

Schaefer, C., Coyne, J. C., \& Lazarus, R. S. (1981). The health-related functions of social support. Journal of Behavioral Medicine, 4, 381-406. 
Schauer, E., Neuner, F., Elbert, T., Ertl, V., Onyut, L. P., Odenwald, M., \& Schauer, M. (2004). Narrative exposure therapy in children: a case study. Intervention, 2, 18-32.

Scholte, W. F., Olff, M., Ventevogel, P., De Vries, G., Jansveld, E., \& Cardozo, B. L. (2004). Mental health symptoms following war and repression in Eastern Afghanistan. The Journal of the American Medical Association, 292, 585-593.

Schweitzer, R., Greenslade, J., \& Kagee, A. (2007). Coping and resilience in refugees from the Sudan: A narrative account. Australian and New Zealand Journal of Psychiatry, 41, 282-288.

Schweitzer, R., Melville, F., Steel, Z., \& Lacherez, P. (2006). Trauma, post-migration living difficulties, and social support as predictors of psychological adjustment in resettled Sudanese refugees. Australian and New Zealand Journal of Psychiatry, 40, 179-187.

Segal, Z. V., Williams, J. M. G., \& Teasdale, J. D. (2002). Mindfulness-based Cognitive Therapy for Depression A New Approach to Preventing Replase. New York, NY: Guilford Press.

Seidman, E., Allen, L., Aber, J., L., Mitchell, C., Feinman, J., Yoshikawa, H., . . Roper, G. C. (1995) Development and validation of adolescentperceived microsystem scales: Social support, daily hassles, and involvement. American Journal of Community Psychology, 23, 355-388.

Seidman, E., Allen, L., Aber, J. L., Mitchell, C., \& Feinman, J. (1994). The impact of school transitions in early adolescence on the self-system and perceived social context of poor urban youth. Child Develop., 65, 507-522.

Shapiro, E. R. (1995). Grief and Trauma in Family: Developmental and Cultural Context. Clinical Quarterly, $5(2-3), 1-6$

Sharma, S., Parnian, S., \& Spielberger, C. D. (1983). A cross-cultural study of the test anxiety levels in Iranian and Indian students. Personality and Individual Differences, 4, 117-120.

Sherina, M. S., Rampal, L., \& Kaneson, N. (2004a). Psychological stress among undergraduate medical students. Medical Journal of Malaysia, 59, 207-211.

Sherina, M. S., Rampal, L., \& Kaneson, N. (2004b). Psychological stress among undergraduate medical students. Medical Journal of Malaysia, 59, 207-211.

Shoeb, M., Weinstein, H., \& Mollica, R. (2007). The Harvard Trauma Questionnaire: Adapting a crosscultural instrument for measuring torture, trauma and posttraumatic stress disorder in Iraqi refugees. International Journal of Social Psychiatry, 53, 447-463.

Silove, D. (1999). The psychological effects of torture, mass human rights violations, and refugee trauma. Toward a conceptual framework. Journal of Nervous \& Mental Disease, 187, 200-207.

Silove, D., Manicavasagar, V., Mollica, R., Thai, M., Khiek, D., Lavelle, I., \& Tor, S. (2007). Screening for deprression and PTSD in a Cambodian Population unaffected by war. The Journal of Nervous and Mental Disease, 195, 152-157.

Silove, D., Steel, S. D., McGorry, P., \& Mohan, P. (1998). Trauma exposure, post-migration stressors, and symptoms of anxiety, depression and posttraumatic stress disorder in Tamil asylum-seekers; comparison with refugees and immigrants. Acta Psychiatrica Scandinavica, 97, 175-181.

Simich, L., Este, D., \& Hamilton, H. (2010). Meanings of home and mental well-being among Sudanese refugees in Canada. Ethnicity and Health, 15, 199-212.

Simich, L., Hamilton, H., \& Baya, B. K. (2006). Mental distress, economic hardships and expectations of life in Canada among Sudanese newcomers. Transcultural Psychiatry, 43, 418-444.

Smith, J. A., Jarman, M., \& Osborn, M. (1999). Doing interpretative phenomenological analysis. In M. Murry, Chamberlain, K. (Ed.), Qualitative Health Psychology (pp. 218-240). London: Sage.

Snyder, C. R., \& Dinoff, B. L. (1999). Coping where have you been? (Vol. I). New York: Oxford University Press.

Somasundaram, D. J., \& Sivayokan, S. (1994). War trauma in a civilian population. British Journal of Psychiatry, 165, 524-527.

Souza, R., Yasuda, S., \& Cristofani, S. (2009). Mental health treatment outcomes in a humanitarian emergency: a pilot model for the integration of mental health into primary care in Habilla, Darfur. International Journal of Mental Health Systems, 3, 1-14.

Sreeramareddy, C. T., Shankar, P. R., \& Binu, V. S. (2007). Psychological morbidity, sources of stress and coping strategies among undergraduate medical students of Nepal. BMC Medical Education, 7.

Stapleton, J. A., Taylor, S., \& Asmundson, G. J. G. (2006). Effects of three PTSD treatments on anger and guilt: exposure therapy, eye movement desensitization and reprocessing, and relaxation training. Journal of Traumatic Stress, 19, 19-28.

Steel, Z., Silove, D., Bird, K., McGorry, P., and Mohan, P. (1999). Pathways from trauma to posttraumatic stress symptoms among tamil asylum seekers, refugees and immigrants. Journal of Traumatic Stress, 12, 421-435. 
Steel, Z., Silove, D., Phan, T., and Bauman, A. (2002). Long-term effect of psychological trauma on the mental health of Vietnamese refugees settled in Australia: A population-based study. Lancet, 360, 1056-1062.

Stoll, K., \& Johnson, P. (2007). Determinants of the psychosocial adjustment of southern Sudanese men. Journal of Refugee Studies, 20, 621-640.

Summerfield, D. (1995). Addressing human response to war and atrocity: Major challenges in research and practices and the limitations of Western psychiatric models. In R. Kleber, C. Figley \& B. Gersons (Eds.), Beyond trauma: Cultural and societal dynamics. New York: Plenum Press.

Swift, D. (1988). Preparing rural students for an urban environment. In ERIC (Ed.), Eric Document Reproduction Service No. ED 296 818. Las Cruces, NM: Clearing House on Rural Education and Small Schools.

Tang, S. S., \& Fox, S. H. (2001). Traumatic Experiences and the Mental Health of Senegalese Refugees. Journal of Nervous and Mental Disease, 189, 507-512.

Tarrier, N., Sommerfield, C., \& Pilgrim, H. (1999). The effect of the relatives' level of expressed emotion (EE) on the outcome of psychological treatment of PTSD patients. Psychological Medicine, 29.

Taylor, S., Thordarson, D. S., Fedoroff, I. C., Maxfield, L., Lovell, K., \& Ogrodniczuk, J. T. (2003). Comparative efficacy, speed, and adverse effects of three PTSD treatments: exposure therapy, EMDR, and relaxation training. Journal of Consulting and Clinical Psychology, 71, 330-338.

Tedeschi, R. G., \& Calhoun, L. G. (1995). Trauma and transformation: growing in the aftermath of suffering. Newbury Park, CA: Sage Publications.

Thabet, A. A., Abed, Y., \& Vostanis, P. (2002). Emotional problems among Palestinian children living in a war zone. The Lancet, 359, 1801- 1804.

Thabet, A. M., \& Vostanis, P. (1999). Post-traumatic stress reactions in children of war. Journal of Child Psychology and Psychiatry, 40, 385-391.

Thapa, S., \& Hauff, E. (2005). Psychological distress among displaced persons during an armed conflict in Nepal. Social Psychiatry and Psychiatric Epidemiology, 40, 672-679.

Thomas, M., \& Choi, J. B. (2006). Acculturative stress and social support among Korean and Indian immigrant adolescents in the United States. Journal of Sociology \& Social Welfare, 33, 123-143.

Timmins, F., \& Kaliszer, M. (2002). Aspects of nurse education programmes that frequently cause stress to nursing students-fact finding sample survey. Nurse Education Today, 22, 203-211.

Tipping, S., Bretherton, D., \& Kaplan, I. (2007). Meaning in life and social connectedness: The experience of Sudanese young people (re)settling in Australia. In D. L. N. Haslam (Ed.), Yearning to breathe free: Seeking asylum in Australia (pp. 157-168). Sydney: The Federation Press.

Tomoda, A., Mori, K., Kimura, M., Takahashi, T., \& Kitamura, T. (2000). One-year prevalence and incidence of depression among first-year university students in Japan: a preliminary study. Psychiatry and Clinical Neurosciences, 54, 583-588.

Torres, l., \& Rollock, D. (2004a). Acculturative distress among Hispanics: The role of acculturation, coping and intercultural competence. Journal of Multicultural Counseling and Development, 32, 155-167.

Torres, l., \& Rollock, D. (2004b). Acculturative distress among Hispanics: The role of acculturation, coping, and intercultural competence. Journal of Multicultural Counseling and Development, 32, 155-167.

Tran, T. V., Manalo, V., \& Nguyen, V. T. D. (2007). Nonlinear relationship between length of residence and depression in a community-based sample of Vietnamese Americans. International Journal of Social Psychiatry, 53, 85-94.

United Nations. (2005). Report of the International Commission of Inquiry on Darfur to the United Nations Secretary-General Pursuant to Security Council Resolution 1564 of 18 September 2004. Geneva, 25 January 2005.

United Nations High Commissioner for Refugees. (2000). Background paper on refugees and asylum seekers from the Sudan. In C. f. D. a. Research (Ed.), UNHCR (Vol. Geneva). Geneva.

Valencia, A. (1994). The attributes of academically successful Mexican-American university male and female students. Journal of Multicultural Counseling and Development, 22, 227-238.

Vaz, R. F., Mbajiorgu, E. F., \& Acuda, S. W. (1998). A preliminary study of stress levels among first year medical student at university of Zimbabwe. Central African Journal of Medicine, 44, 214-219.

Vazquez, L. A., \& Garcia-Vazquez, E. (1995). Variables of success and stress with Mexican American students. College Student Journal, 29, 221-226.

Verdeli, H., Clougherty, K., Bolton, P., Speelman, L., Ndogoni, L., Bass, J., . . Weissman, M. M. (2003) Adapting a group interpersonal psychotherapy for a developing country: experience in rural Uganda. World Psychiatry, 2, 114-120. 
Vergara, M. B., Smith, N., \& Keelea, B. (2010). Emotional Intelligence, Coping Responses, and Length of Stay as Correlates of Acculturative Stress Among International University Students in Thailand. Procedia Social and Behavioral Sciences, 51498-1504.

Wagnild, G. (2008). Resilience among frontier women.

Wagnild, G., \& Young, H. (1993). Development and psychometric evaluation of the Resilience Scale. Journal of Nursing Measurement, 1, 165-178.

Wagnild, G. M. (2009). A Review of Resilience Scale. Journal of Nursing Measurement, 17, 105-113.

Waltz, M. (1994). Social isolation and social mediators of the stress of illness. Medizinsoziologie 2, 188201.

Wei, M., Heppner, P. P., Mallen, M. J., Ku, T. K., Liao, Y., \& Wu, T. (2007). Acculturative stress, perfectionism, years in the United States, and depression among Chinese international students. Journal of Counseling Psychology, 54, 385-394.

Werner, E. (1990). Protective factors and individual resilience. In S. Meisels \& J. Shonkoff (Eds.), Handbook of Early Childhood Intervention. New York, NY: Cambridge University Press.

Werner, E. E. (1995). Resilience in development. Current Directions in Psychological Science, 4, 81-85.

Werner, E. E., \& Smith, R. C. (1992). Overcoming the odds: High risk children from birth to adulthood. New York: Cornell University Press.

Wheaton, B. (2010). The Stress Process as a successful paradigm. In W. R. Avison, C. S. Aneshensel, S. Schieman \& B. Wheaton (Eds.), Advances in the conceptualization of the Stress Process: Essays in honor of Leonard I. Pearlin. New York: Springer.

Whitfield, C. L. (1995). The forgotten difference: Ordinary memory versus traumatic memory. Consciousness and Cognition, 4, 88-94.

Williams, C. L., \& Berry, J. W. (1991). Preliminary prevention of acculturative stress among refugees: Application for psychological theory and practice. American Psychologist, 46, 632-641.

Work, W. C., Parker, G. R., \& Cowen, E. L. (1990). The impact of life stressors on childhood adjustment: Multiple perspectives. Journal of Community Psychology, 18, 73-78.

World Bank. (2005). Poverty Reduction Strategies 2005 Revies: Lessons and resources. . Washington, DC: The World Bank.

World Health Organisation. (1992). ICD 10: Classification of mental and behavioural disorders: Clinical description and diagnostic guidelines. Geneva.

World Health Organisation. (2008). Scientific evidence supports UN OCHA extrapolation on Darfur mortality. In Collaborating Center for research on the Epidemiology of Disasters (Ed.).

World Health Organisation. (2009). Mental Health System in Sudan.

Wortman, C. B., Battle, E. S., \& Lemkau, J. P. (1997). Coming to terms with sudden, traumatic death of a spouse or child. In R.C. Davis \& A. J. Lurigio (Eds.), Victims of crime (Vol. 2, pp. 108-133). Thousands Oaks, CA: Sage Publications.

Wortman, C. B., \& Silver, R. C. (1992). Reconsidering assumptions about coping and loss: An overview of current research. In S. F. L. Montada, and M. J. Lerner (Ed.), Life crisis and experiences of loss in adulthood (pp. 341-365). Hilsdale, NJ: Erlbaum.

Wyman, P. A., Cowen, E. L., Work, W. C., \& Parker, G. R. (1991). Developmental and family milieu correlates of resilience in urban children who have experienced major life stress. American Journal of Community Psychology, 19, 405-426.

Ye, J. (2005). Acculturative stress and use of the internet among East Asian international students in the United States. Cyber Psychology \& Behavior, 8, 154-161.

Young, A. (1995). The Harmony of Illusions: Inventing Post-Traumatic Stress Disorder: Princeton University Press.

Young, G. (1992). Chicana college students on the Texas-Mexico Border: Tradition and transformation. Hispanic Journal of Behavioral Sciences, 14, 341-352.

Young, J., Osman, A. M., Akilu, Y., Dale, R., Badri, B., \& Faddle, A. J. A. (2005). Darfur-Livelihoods under siege. Medford, MA, USA: Tufts University.

Zea, M. C., Jarama, S. L., \& Bianchi, F. T. (1995). Social and psychosocial competence: Explaining the adaptation to college of ethnically diverse students. American Journal of Community Psychology, 23 509-531. 


\section{About the Author}

Born in Khartoum, Sudan on the $5^{\text {th }}$ of August 1968, Alia lived much of her formative years outside her motherland, only to return to complete her education at Unity High School in 1984 and Ahfad University for Women in 1986. Graduating from the School of Psychology and Pre-school Education, Alia then went back to the UK in 1992 to join the University of Warwick for her Masters of Science Degree in Psychology. Immediately after graduating, she started her career as a lecturer in all fields of Psychology, picking up varied certificates along the way, such as, 'training of group leaders and trainers', 'women's empowerment and gender development', and 'professionalization of teaching skills for higher education staff'.

Initiating the Counseling and Health Psychology sub-specialization in the School of Psychology in 1994, Alia held the position of head coordinator and curricula design and development, producing course outlines, teaching materials, lecturer instructors' manuals and student's study guide, and training guidebooks for over twenty new courses. By the end of 1994 she taught as well as oversaw that all therapy approaches, counselor skills training and capacity building of the first batch of Sudanese women counselors from AUW was carried out. Thereafter she continued her career at AUW until her departure once again from the Sudan in 1998.

Alia enjoyed the challenges of motherhood as well as continuing to hold lecturing positions during the early to mid 2000s, such as, the Arab Open University in Kuwait, and Abu Dhabi University in the UAE, as well as becoming an affiliate employee counselor with the UK-based Personal Performance Consultants International (PPCI).

In August of 2008, Alia travelled to Maastricht University, where she began with a course on planning health promotion programs through intervention mapping. The following year she initiated her PhD study at the School for Public Health and Primary Care: CAPHRI, Department of Health Promotion. During the next three years, Alia would travel back and forth from her Abu Dhabi residence to UM and AUW on a yearly basis as she conducted the research in this dissertation.

Following her graduation in 2013, Alia has joined the University of Western in London Ontario, where there is a Memorandum of Understanding with AUW for staff and student exchange. She will then return to AUW to take up the position of lecturer and trainer in the newly established National trauma treatment and training center of AUW, of which her past endeavours and this dissertation played a key role in its origin. 
Amongst her many unpublished works as part of her participation in workshops and conferences as well as contributions to the field of women, gender and psychology in UN organizations and NGO, Alia has made the following publications:

Badri, A., An Experimental Comparison of Sudanese Male Children's Cultural Orientation and Intelligence (Draw - A - Man Test), Ahfad Journal, Vol. 7, No. 1, 1990.

Badri, A., Women and Peace Dialogue: Northern and Southern Women Meet, Women Magazine, BBSAWS, issue 10, December 1996.

Badri, A., The Challenges of the Working Women, ElNisf AlWaid Magazine, Ahfad University for Women, issue 33, July 2003.

Badri, A., Crutzen, R., \& Van den Borne, H. W. (2012). Experiences and psychosocial adjustment of Darfuri female students affected by war: An exploratory study. International Journal of Psychology, e-pub ahead of print.

Badri, A., Crutzen, R., \& Van den Borne, H. W. (2012). Exposures to war-related traumatic events and post-traumatic stress disorder symptoms among displaced Darfuri female university students: An exploratory study. BMC Public Health, 12, 603.

\author{
Alia Badri \\ 262 Skyline Ave \\ London ON \\ N5X 0A4 \\ Canada \\ +12269271968 \\ aliabadri@hotmail.com
}

